\title{
Tuning phonon properties in thermoelectric materials
}

\section{G. P. Srivastava}

School of Physics, University of Exeter, Stocker Road, Exeter EX4 4QL, UK

E-mail: G.P.Srivastava@exeter.ac.uk; gps@excc.ex.ac.uk

\begin{abstract}
.
This review article presents a discussion of theoretical progress made over the past several decades towards our understanding of thermoelectric properties of materials. Particular emphasis is paid towards describing recent progress in 'tuning' phonon properties of nano-composite materials for gaining enhancement of the thermoelectric figure of merit.
\end{abstract}




\section{Introduction}

Phonons are zero-spin bosonic quanta of elementary excitations arising from the collective simple harmonic oscillations of atoms in crystalline solids. There is increasing interest in the theoretical prediction of material properties in which phonons play an essential role. In particular, it is becoming clear that phonon properties of nanostructured materials can be 'tuned' to a large degree for future technological applications.

We consider here thermoelectricity (TE), the process of generating either electricity from heat engines or heating devices from electricity. Examples of modern TE applications include portable refrigerators, beverage coolers, electronic component coolers, infrared sensing, etc. Possible future applications of TE devices include efficient conversion of waste heat (e.g. from waste and during powering of vehicles, etc) into usable energy, improving efficiency of photovoltaic cells, etc. The efficiency of a thermoelectric device is often expressed in terms of the dimensionless figure of merit $Z T$ of the material defined as

$$
Z T=\frac{S^{2} \sigma T}{\kappa},
$$

where $S$ is the absolute thermopower, or thermopower, or Seebeck coeffient, $\sigma$ is the electrical conductivity, $T$ is the average device temperature in Kelvin, and $\kappa$ is the total thermal conductivity. The efficiency of a thermoelectric generator $\left(\eta_{\mathrm{TE}}\right)$ is defined as

$$
\eta_{\mathrm{TE}}=\frac{T_{h}-T_{c}}{T_{h}} \frac{\sqrt{1+Z T}-1}{\sqrt{1+Z T}+\frac{T_{c}}{T_{h}}},
$$

where $T_{h}\left(T_{c}\right)$ is the temperature at the hot(cold) side, and $T$ is the average temperature between $T_{h}$ and $T_{c}$. Similarly, the coefficient of performance $\left(\Phi_{\mathrm{TE}}\right)$ of a single-stage thermoelectric cooler is defined as

$$
\Phi_{\mathrm{TE}}=\frac{T_{c}}{T_{h}-T_{c}} \frac{\sqrt{1+Z T}-\frac{T_{h}}{T_{c}}}{\sqrt{1+Z T}+1} .
$$

The basic principles (including the derivation of Eqs. (1) and (2)) and applications of thermoelectrics have been thoroughly discussed in a number of books and review articles over the past six decades (the list is too large to include here, but for a few non-biased references on books see: in 1950s [1], in 1960s [2, 3, 4], in 1980s [5], in 2000s $[6,7,8,9]$, and similarly for a few non-biased references on review articles see: in 1950s [10], in 1960s [11], in 1970s [12, 13], in 1980s [14], in 1990s [15, 17], in 2000s $[18,19,20,21,22,23,24,25])$. The review article by Bulusu and Walker [21] has summarised the progression of thermoelectricity and the various models for predicting thermoelectric performance since the discovery of Seebeck effect in 1821 until the early 1990s. A recent review by Maldovan [26] explains that understanding and controlling the phononic (i.e. phonon blocking) properties of materials may also provide opportunities for TE applications.

Equation (2) suggests that the efficiency of a thermoelectric device operating between $T c=300 \mathrm{~K}$ and $T_{h}=700 \mathrm{~K}$ would be $12.84 \%, 19.36 \%, 23.53 \%, 26.51 \%$ 
and $28.78 \%$ for $Z T$ values of $1.0,2.0,3.0,4.0$ and 5.0, respectively. The central issue in thermoelecrics, obviously, then is to find or design materials that are characterised by a large figure of merit $Z T$. Estimates and experimental measurements of $Z T$ for different classes of materials (metals, semiconductors, insulators) have firmly pointed out that the best $Z T$ materials are doped semiconductors. In his review article in 1984, Wood [14] concluded that '.. although the experimental results to date indicate that $Z T \sim 1$ represents an upper limit, there is no theoretical basis for this conclusion for either the broad- or narrow-band semiconductors'. Vining in 1992 [15] stated 'Neither thermodynmics nor irreversible thermodynamics, however, place any upper limit on ZT and transport theory reveals nothing special about the region $Z T \sim 1$. .. Today, the performance of thermoelectric devices is limited to a small fraction ( $10 \%$ ) of the Carnot limits because for available thermoelectric materials $Z T \leq 1$. ... in the long run there ... appear to be only two real possibilities: 1) $Z T>>1$ is possible and will be demonstrated experimentally, or 2) $Z T \sim 1$ represents some as yet unstated principle.' Mahan [16] derived an expression for maximum $Z T$ in TE applications. His theory shows that the semiconductor band gap for large $Z T$ should be $10 k_{\mathrm{B}} T(0.25 \mathrm{eV}$ at room temperature). With an appropriate choice of parameters for good TE materials, his theory predicts the maximum value of $Z T$ to be about 1-2. The review article in 1999 by DiSalvo [17] summarises: '... There are theoretical grounds for some optimism that materials with $Z T>4$ will be found. However, the current inability to predict new structures and compositions of new solid-state compounds makes the search for more-efficient materials non-systematic, ... Hopefully, the advances will come soon.' However, no bulk material has been found to really match the optimism expressed in these articles. After a long period of quiescence, the development of techniques to produce high quality nanostructures has led to the resurgence of experimental work for investigating low-dimensional and nanocomposite materials that might be characterised by commercially viable enhanced values of $Z T$ beyond unity.

What are the factors that help enhance $Z T$ ? This has been the topic of much research over the years, as is evident from almost all the review articles cited in this article. The numerator in Eq. (1) can be expressed as $P F \times T$, where $P F=S^{2} \sigma$ is the power factor. Generally $S^{2}$ decreases and $\sigma$ increases with the carrier concentration in a semiconductor. However the decrease in $S^{2}$ is much smaller than the increase in $\sigma$. Consequently, the power factor peaks for a certain value of carrier concentration, typically in the range $10^{25}-10^{26} \mathrm{~m}^{-3}$. The maximum value of $P F$ in a doped bulk semiconductor is limited by the operating temperature and some fixed, intrinsic properties related to its electronic band structure (see discussions in Refs [14, 22]). In a semiconducting material thermal conductivity $\kappa$ is contributed mainly by carriers $\left(\kappa_{\text {carriers }}\right)$ and phonons (lattice contribution $\kappa_{\mathrm{ph}}$ )

$$
\kappa=\kappa_{\text {carriers }}+\kappa_{\text {ph }} .
$$

The contribution $\kappa_{\text {carriers }}$ mainly comes from monopolar carriers (electrons or holes $\kappa_{\text {mp }}$ ) in the extrinsic regime and bipolar carriers (electrons and holes $\kappa_{\mathrm{bp}}$ ) in the intrinsic 
regime:

$$
\kappa_{\text {carriers }}=\kappa_{\mathrm{mp}}+\kappa_{\mathrm{bp}}
$$

The monopolar carrier contribution to the thermal conductivity $\kappa_{\mathrm{mp}}$ is related to the electrical conductivity $\sigma$ through the Wiedemann-Franz relationship, and the overall carrier contribution $\kappa_{\text {carriers }}$ is generally much smaller than the lattice thermal conductivity $\kappa_{\mathrm{ph}}$ at temperatures below which the semiconductor becomes intrinsic in its electrical nature. The phonon contribution $\kappa_{\mathrm{ph}}$, on the other hand, can be governed by both intrinsic factors (e.g. structure and atomic masses of species) and extrinsic factors (e.g. amount and atomic mass of dopant and temperature).

It has been maintained [27] that the major factor which determines the optimum value of $Z T$ for a given semiconductor is the quantity $F$, given as

$$
F=\left(m^{*}\right)^{3 / 2} \mu / \kappa_{\mathrm{ph}},
$$

where $m^{*}$ is the carrier density-of-states effective mass and $\mu$ is the carrier mobility. The dependence of the high-temperature lattice thermal conductivity of semiconductors and insulators on material parameters has been examined by several workers. We will quote results obtained by a few groups. Leibfried and Schlömann [28] showed that

$$
\kappa_{\mathrm{ph}} \propto A V_{a}^{1 / 3} \theta_{D}^{3} T^{-1},
$$

where $T$ is the temperature, $\theta_{D}$ is the Debye temperature, $V_{a}$ is the mean atomic volume and $A$ is the mean atomic weight. Keyes [29] predicted the following relationship:

$$
\kappa_{\mathrm{ph}} \sim T_{m}^{3 / 2} \varrho^{2 / 3} A^{-7 / 6} T^{-1},
$$

where $T_{m}$ is the melting temperature and $\varrho$ is the material density. Goldsmid [27], on the other hand, obtained the following simple relationship:

$$
\kappa_{\mathrm{ph}} \propto(\varrho / A)^{1 / 3} \theta_{D}
$$

The ratio $\varrho / A$ tends to fall rather slowly with $A$. However, Goldsmid argued that a high Debye temperature $\theta_{D}$ and a high mean atomic weight $A$ are important considerations for low values of $\kappa_{\mathrm{ph}}$.

A limited amount of reduction in $\kappa_{\mathrm{ph}}$ can be achieved by considering phases of a single-crystal material with complex structural units. As a rough estimate, $\kappa_{\mathrm{ph}} \propto n^{-2 / 3}$, where $n$ represents the number of atoms in a unit cell [30]. Alloy formation by mixing two or more materials of different atomic masses allows for greater reduction in $\kappa_{\mathrm{ph}}[14,31]$. A material prepared using techniques, such as random alloying, which produces glass-like hugely reduced $\kappa_{\mathrm{ph}}$ without significantly reducing the power factor $P F$ and hence helps enhance $Z T$, is generally known as the phonon-glass electron-crystal (PGEC) [9, 32]. Experimental techniques are now ripe for fabrication of nanocomposites with rough or disordered interfaces which hold greater promise for reduced $\kappa_{\mathrm{ph}}$ and thus enhanced $Z T$.

To help experimental work in the field of thermoelectricity it is important to develop adequate theories that can make confident predictions of phonon properties. This review article will present a discussion of the theoretical progress made towards 
our understanding of thermoelectric properties of single-crystal and nanostructured semiconductors, with an emphasis on recent $a b$ initio attempts. We will first present a brief theoretical description of the terms $S, \sigma$ and $\kappa_{\text {carriers. }}$ The rest of this article will then discuss, using the predictive power of theory, how phonon properties (e.g. dispersion relation, velocity, lifetime, and hence $\kappa_{\mathrm{ph}}$ ) can be 'tuned' by selective choice of material composition, structure and physical parameters to achieve enhancement in the thermoelectric figure of merit $Z T$ of nanocomposite materials.

\section{Transport coefficients}

Under the applications of a small electric field $\boldsymbol{E}$ and temperature gradient $\boldsymbol{\nabla} T$, phenomenological expressions for the electrical current $\boldsymbol{J}$ and thermal current $\dot{\boldsymbol{Q}}$ can be written as

$$
\begin{aligned}
& \boldsymbol{J}=L_{E E} \boldsymbol{E}+L_{E T} \boldsymbol{\nabla} T, \\
& \dot{\boldsymbol{Q}}=L_{T E} \boldsymbol{E}+L_{T T} \boldsymbol{\nabla} T,
\end{aligned}
$$

where the coefficients $L_{E E}, L_{E T}, L_{T E}$ and $L_{T T}$ are in general tensors, with $L_{E T}$ and $L_{T E}$ being inter-related through the thermodynamic Kelvin-Onsager relation $L_{E T}=$ $-L_{T E} / T$. These are related to the TE coeffcients as follows

$$
\sigma=L_{E E}, \quad S L_{E E}=-L_{E T}, \quad \kappa L_{E E}=-\left(L_{T T} L_{E E}-L_{T E} L_{E T}\right) .
$$

It is useful to define another transport coefficient $\chi$

$$
\chi=-L_{T T}=\kappa+S^{2} \sigma T .
$$

The Lorenz number $\mathcal{L}$ can be obtained from the relation

$$
\mathcal{L} T L_{E E} L_{E E}=-L_{T T} L_{E E}+L_{T E} L_{E T} \text {. }
$$

The coefficients $\kappa$ and $\chi$ represent the thermal conductivities, respectively, under the zero electric current and zero electric field conditions.

The TE coefficients for composite systems can be expressed in terms of their constituents. Consider a superlattice (SL) ...A/B/A/B/... made of a periodic arrangement of layers of materials $\mathrm{A}$ and $\mathrm{B}$, where $\mathrm{A}$ is a quantum well of width $d_{A}$ and $\mathrm{B}$ is a barrier of width $d_{B}$. Bergman and Levy [33] provided a formulation for expressing the TE transport coeffcients for two-component composites in which both components are isotropic. Their formulation was followed by Lin-Chung and Reinecke [34] to study the TE properties of superlattice structures. Following Lin-Chung and Reinecke [34], we can express

in-plane coefficients:

$$
\sigma_{\mathrm{SL}}^{\mathrm{in}}=\frac{d_{A} \sigma_{A}+d_{B} \sigma_{B}}{d_{A}+d_{B}}, \quad \chi_{\mathrm{SL}}^{\mathrm{in}}=\frac{d_{A} \chi_{A}+d_{B} \chi_{B}}{d_{A}+d_{B}}, \quad S_{\mathrm{SL}}^{\mathrm{in}}=\frac{d_{A} \sigma_{A} S_{A}+d_{B} \sigma_{B} S_{B}}{d_{A} \sigma_{A}+d_{B} \sigma_{B}} .
$$

cross-plane coefficients:

$$
\sigma_{\mathrm{SL}}^{\text {cross }}=\frac{\sigma_{A} \kappa_{A}}{d_{A}} A_{\mathrm{SL}} W_{\mathrm{SL}}, \quad \gamma_{\mathrm{SL}}^{\mathrm{cross}}=\frac{\sigma_{A} \kappa_{A}}{d_{A}} B_{\mathrm{SL}} W_{\mathrm{SL}}, \quad S_{\mathrm{SL}}^{\mathrm{cross}}=\frac{\sigma_{A} S_{A}+q_{\mathrm{SL}} \sigma_{B} S_{B}}{\sigma_{A}+q_{\mathrm{SL}} \sigma_{B}},
$$


where

$$
\begin{aligned}
& W_{\mathrm{SL}}=\left(d_{A}+d_{B}\right)\left[\left(\chi_{A}+q_{\mathrm{SL}} \chi_{B}\right)\left(\sigma_{A}+q_{\mathrm{SL}} \sigma_{B}\right)-T\left(\sigma_{A} S_{A}+q_{\mathrm{SL}} \sigma_{B} S_{B}\right)^{2}\right]^{-1} \\
& q_{\mathrm{SL}}=\frac{d_{B} \sigma_{A} \kappa_{A}}{d_{A} \sigma_{B} \kappa_{B}}, \quad A_{\mathrm{SL}}=\left(\sigma_{A}+q_{\mathrm{SL}} \sigma_{B}\right), \quad B_{\mathrm{SL}}=\left(\chi_{A}+q_{\mathrm{SL}} \chi_{B}\right) .
\end{aligned}
$$

\section{Carrier components of the transport coefficients}

Let us denote the occupation distribution function of carriers as $f[E(\boldsymbol{k}), \boldsymbol{r}]$, with $\boldsymbol{r}$, $\boldsymbol{k}$ and $E(\boldsymbol{k})$ being space position, wavevector and energy, respectively. Assuming the validity of the Boltzmann equation satisfied by $f$, we can express $[2,35]$

$$
\begin{aligned}
& \left(L_{\mathrm{EE}}\right)_{i j}=\mathcal{Q}^{2} \sum_{\boldsymbol{k}} v_{\boldsymbol{k}}^{i} v^{j} \boldsymbol{k}^{\tau} \boldsymbol{\boldsymbol { k }} \frac{\partial \bar{f}_{\boldsymbol{k}}}{\partial E_{\boldsymbol{k}}},
\end{aligned}
$$

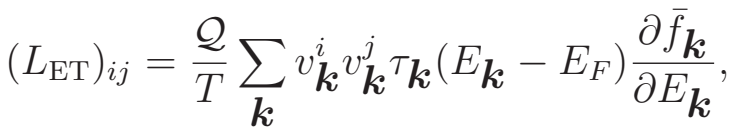

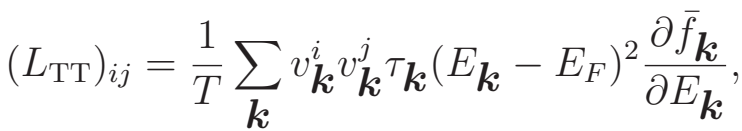

where $\mathcal{Q}=-e(e)$ is the carrier [electron(hole)] charge, $\boldsymbol{k}$ represents the carrier wavevector, $v_{\boldsymbol{k}}^{i}=\frac{\partial E \boldsymbol{k}}{\partial k_{i}}$ is the $i^{\text {th }}$ component of the carrier velocity, $\tau_{\boldsymbol{k}}$ is the carrier relaxation time, $E_{F} \equiv E_{F}(T)$ represents the Fermi level and $\bar{f}_{\boldsymbol{k}}$ is the Fermi-Dirac equilibrium distribution function. With these, the carrier components of the transport coeffcients can be expressed as

$$
\begin{aligned}
\sigma_{i j} & =\left(L_{E E}\right)_{i j}, \\
S_{i j} & =-\left(L_{E E}^{-1}\right)_{i l}\left(L_{E T}\right)_{l j}, \\
\left(\kappa_{\text {carriers }}\right)_{i j} & =\left(L_{E E}^{-1}\right)_{i l}\left(L_{E T}\right)_{l k}\left(L_{T E}\right)_{k j}-\left(L_{T T}\right)_{i j} .
\end{aligned}
$$

It is clear from Eqs. (17 - 22) that for calculating the transport coefficients we require (i) knowledge of the full electronic band structure, (ii) knowledge of the carrier lifetime, (iii) a realistic method of performing Brillouin zone summation over the wavevector $\boldsymbol{k}$ and (iv) temperature dependence of the Fermi level. Essentially, each of the four items is a very demanding job, theoretically and computationally, and simplified approaches are usually adopted to deal with these. Items (i) and (iii) can normally be calculated directly, and then first-order time-dependent perturbation theory can be applied to obtain expressions for carrier lifetime due to scattering from appropriate sources, such as ionised impurities, acoustic phonons and optical phonons. Using Matthiessen's rule the total contribution to the lifetime is obtained as $\tau_{\boldsymbol{k}}^{-1}=\sum_{j} \tau_{\boldsymbol{k}, \text {, }}^{-1}$, where the index $j$ indicates a scattering process. In the following subsections we briefly describe the usually adopted simplified approaches.

We will represent system dimensionality with $D(D=3$ for bulk and $D<3$ for low-dimensional structures). In particular, we will consider a low-dimensional system comprised of materials $\mathrm{A}$ and $\mathrm{B}$, with $\mathrm{A}$ being characterised with a single confined energy state inside a square quantum well of width $d_{A}$. 


\subsection{Temperature dependence of the Fermi level}

In order to evaluate the transport coefficient $S, \sigma$ and $\kappa_{\text {carriers }}$ it is important to establish the temperature dependence of the Fermi level. In principle, the Fermi level at temperature $T$ for a semiconductor can be computed from the requirement of its electrical neutrality and the full knowledge of electronic band structure (see, e.g. [36]), and using a root-finding technique such as the Van-Wjingaarden-Decker-Brent method [37]. It is well known that straight-forward density-functional-based first-principles electronic band structure are both computationally demanding and suffer from the band-gap problem. However, for transport calculations it is often adequate to consider the band structure of the semiconductor within the nearly-free-electron approximation, with non-degenerate conduction and valence bands characerised with density-of-states effective masses $m_{n}^{*}$ and $m_{p}^{*}$, respectively. At temperatures of interest, the separation of the electron and hole energies from the Fermi level satisfies $\left|E-E_{F}\right|>>k_{B} T$ and the Fermi-Dirac distribution funtion can be approximated by the Boltzmann distribution function. The electrical neutrality condition for an n-type semiconductor can be written as

$$
0=N_{d}^{\text {ion }}-n_{0}+p_{0}
$$

where $N_{d}^{\text {ion }}, n_{0}$ and $p_{0}$ are the concentrations of ionised donors, thermally excited electrons and thermally excited holes, respectively. Following Refs. [22, 38, 39] and with the considerations mentioned above, we can express

$$
\begin{aligned}
N_{d}^{\text {ion }} & =\frac{N_{d}}{1+2 e^{-\left(E_{d} / k_{\mathrm{B}} T\right)-\eta_{R}}}, \\
n_{0} & =\frac{N_{\text {valleys }}}{g_{D} d_{A}^{3-D}}\left(\frac{2 k_{\mathrm{B}} T m_{n}^{*}}{\hbar^{2}}\right)^{D / 2} \mathcal{F}_{D / 2-1}\left(\beta_{n}\right), \\
p_{0} & =\frac{N_{\text {valleys }}}{g_{D} d_{A}^{3-D}}\left(\frac{2 k_{\mathrm{B}} T m_{p}^{*}}{\hbar^{2}}\right)^{D / 2} \mathcal{F}_{D / 2-1}\left(\beta_{p}\right) .
\end{aligned}
$$

Here $N_{d}$ is the concentration of donor impurity atoms, $N_{\text {valleys }}$ is the number of degenerate energy valleys, $E_{d}$ is the donor energy level, $E_{c}$ and $E_{v}$ are the conduction and valence band edges, $m_{n}^{*}$ is electron effective mass, $m_{p}^{*}$ is hole effective mass, $g_{D}$ takes values $2 \pi^{2}, 2 \pi$ and $\pi$ for $D=3,2$ and 1 , respectively,

$$
\begin{aligned}
& E_{1}^{n(p)}=\hbar^{2} \pi^{2} / 2 d_{A}^{2} m_{n(p)}^{*}, \\
& \left.\beta_{n}=\zeta-\left(\left(E_{c}+(3-D) E_{1}^{n}\right) / k_{\mathrm{B}} T\right), \quad \beta_{p}=\left(E_{v}-(3-D) E_{1}^{p}\right) / k_{\mathrm{B}} T\right)-\zeta,
\end{aligned}
$$

$\zeta=E_{F} / k_{\mathrm{B}} T$ being the reduced Fermi energy, and $\mathcal{F}_{n}(y)$ is the Fermi integral

$$
\mathcal{F}_{n}(y)=\int_{0}^{\infty} \frac{x^{n}}{e^{x-y}+1} \mathrm{~d} x
$$

Using one donor impurity level, two bulk bands (one conduction and one valence) with a band gap $E_{g}$ and employing the nearly free electron model, Hutner et al [40] expressed Eq. (23) in the form

$$
\frac{2 \mathcal{P}}{\sqrt{\pi}} \mathcal{F}_{1 / 2}(\zeta)=\frac{n_{d} \exp \left(-\zeta_{d}\right)}{\exp (\zeta)+\exp \left(-\zeta_{d}\right)}+\frac{2 \mathcal{R}}{\sqrt{\pi}} \mathcal{F}_{1 / 2}\left(-\zeta-\zeta_{g}\right)
$$


where $n_{d}$ is the concentration of donor states (number of electrons per unit volume occupying the donor levels), $E_{d}$ is the position of the donor level below the conduction band $E_{c}, \zeta_{d}=\left(E_{c}-E_{d}\right) / k_{B} T, \zeta_{g}=E_{g} / k_{B} T$ and

$$
\mathcal{P}=2\left(2 \pi m_{n}^{*} k_{B} T\right)^{3 / 2} / h^{3}, \quad \mathcal{R}=2\left(2 \pi m_{p}^{*} k_{B} T\right)^{3 / 2} / h^{3} .
$$

This is much easier to solve numerically than Eq. (23) using the full band structure.

Simpler schemes leading to analytical expressions for the Fermi level (chemical potential, or temperature dependent Fermi energy) in bulk semiconductors have been discussed in the book by McKelvey [39]. Three different situations can be considered: (i) strictly extrinsic regime with incomplete ionisation of impurity levels (realised at low temperatures), (ii) intrinsic regime with equal hole and electron concentrations from the host material, and (iii) a general case for all but the lowest temperatures and the highest values of impurity concentrations (so that $k_{B} T>>E_{d}$ and Boltzmann distribution function can be used) incorporating ionisation of impurity levels as well as excitations of bulk electron and holes. We will reproduce the useful results below.

\section{(i) Incomplete ionisation of impurity levels:}

At very low temperatures donor and acceptor levels may not be completely ionised. In such an extrinsic regime for a $D$-dimensional semiconductor, under the n-type condition $n_{0}-N_{d}+n_{d}=0$, or the p-type condition $n_{0}-N_{a}+n_{d}=0$, the temperature dependence of the Fermi level can be expressed analytically (see, e.g. [22, 39]):

$$
\begin{aligned}
& \text { for } \mathrm{n}-\text { type : } \\
& E_{F}^{\mathrm{ext}, \mathrm{n}}=\quad \frac{\zeta_{i}^{n} k_{B} T}{2}+\frac{k_{\mathrm{B}} T}{2} \ln \frac{N_{\mathrm{d}}}{2 U_{\mathrm{c}}}-k_{\mathrm{B}} T \sinh ^{-1}\left[\sqrt{\frac{U_{\mathrm{c}}}{8 N_{\mathrm{d}}}} \exp \left(-\zeta_{i}^{\mathrm{n}} / 2\right)\right], \\
& \text { for } \mathrm{p}-\text { type : } \\
& E_{F}^{\mathrm{ext}, \mathrm{p}}=\quad \frac{\zeta_{i}^{p} k_{B} T}{2}+\frac{k_{\mathrm{B}} T}{2} \ln \frac{N_{\mathrm{a}}}{2 U_{\mathrm{v}}}-k_{\mathrm{B}} T \sinh ^{-1}\left[\sqrt{\frac{U_{\mathrm{v}}}{8 N_{\mathrm{a}}}} \exp \left(-\zeta_{i}^{\mathrm{p}} / 2\right)\right],
\end{aligned}
$$

where $\zeta_{i}^{\mathrm{n}}=\left[E_{c}-E_{d}+(3-D) E_{1}^{n}\right] / k_{B} T$ is the reduced donor ionisation energy, $\zeta_{i}^{\mathrm{p}}=\left[E_{a}-E_{v}+(3-D) E_{1}^{p}\right] / k_{B} T$ is the acceptor ionisation energy, and $U_{c(v)}=$ $2\left(2 \pi m_{n(p)}^{*} k_{\mathrm{B}} T / h^{2}\right)^{3 / 2}$. In the intrinsic regime, under the condition $n_{0}=p_{0}$,

$$
E_{F}^{\mathrm{int}}(T)=\frac{1}{2}\left[E_{c}+(3-D) E_{1}^{n}+E_{v}-(3-D) E_{1}^{p}\right]+\frac{3}{4} k_{B} T \ln \left(\frac{m_{p}^{*}}{m_{n}^{*}}\right) .
$$

(ii) Case of completely ionised impurities:

The Fermi level for an n-type semiconductor with completely ionised donors and in the temperature range over which the Boltzmann distribution function can be used, the following result can be derived [39]

$$
E_{F}(T)=\frac{1}{2}\left(E_{v}+E_{c}\right)+\frac{3}{4} k_{\mathrm{B}} T \ln \left(\frac{m_{p}^{*}}{m_{n}^{*}}\right)+k_{\mathrm{B}} T \sinh ^{-1}\left(\frac{N_{d}}{2 \sqrt{U_{c} U_{v}} \exp \left(-\zeta_{g}\right)}\right) .
$$

This is the case that applies at all but the lowest temperatures and the highest values of impurity concentrations. At high temperatures the number of thermally excited 
carriers (electrons and holes) becomes far in excess of the donors and the result in Eq. (33) reduces, approximately, to the result obtained from Eq. (32).

Figure 1 shows the temperature dependence of the Fermi energy for an n-doped semiconductor with considerations of: carrier concentration $N_{d}=1.0 \times 10^{26} \mathrm{~m}^{-3}$, band gap of $0.75 \mathrm{eV}$, donor energy level $5 \mathrm{meV}$ below the conduction band edge, and the ratio of hole to electron density-of-states effective masses taken as 1.2. The dashed curve (plotted from Eq. (31) is valid only at low temperatures when the system is strictly extrinsic, while the dotted curve (plotted from Eq. (32) is valid for high temperature when the system the purely intrinsic. The solid curve (plotted from Eq. (33) shows the results for the realistic n-type system. At zero temperature, the Fermi energy lies at $E_{c}-2.5 \mathrm{meV}$, midway between the donor level and the conduction band edge. After an initial slight rise, $E_{F}$ for the extrinsic system decreases as temperature increases, and then increases with temperature above approximately $1000 \mathrm{~K}$ when the system behaves like an intrinsic semiconductor.

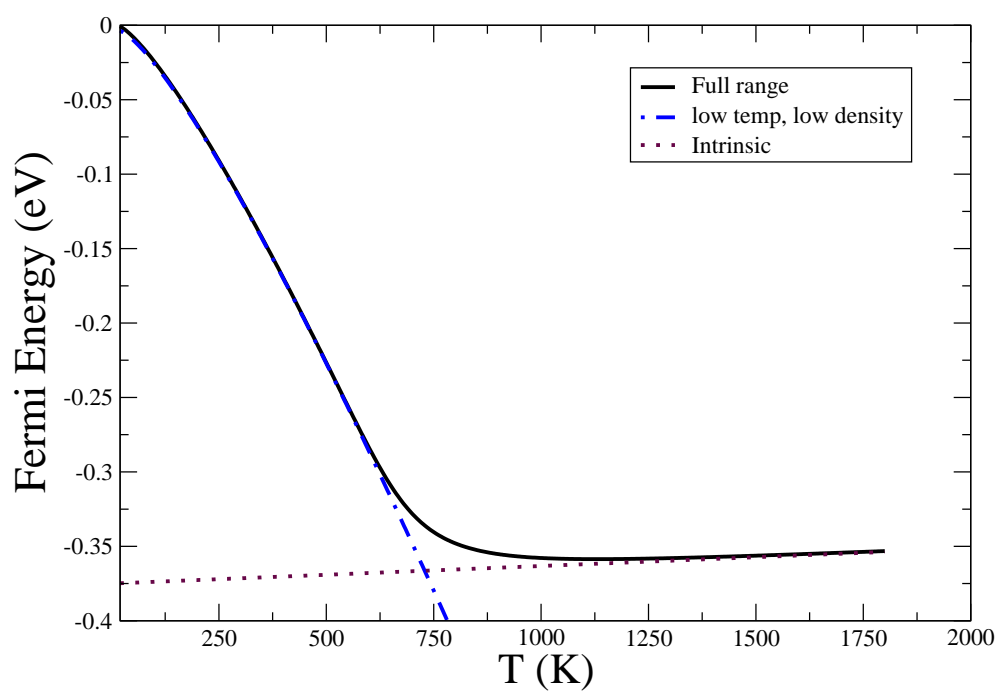

Figure 1. Temperature variation of Fermi energy in n-type semiconductor computed for the donor concentration $N_{d}=1.0 \times 10^{26} \mathrm{~m}^{-3}$. The conduction band edge, donor level and the valence band edge are set at $E_{c}=0, E_{d}=E_{c}-5 \mathrm{meV}, E_{v}=E_{c}-0.75$ $\mathrm{eV}$, and the ratio of hole and electron density-of-states effective mass is taken as $m_{p}^{*}(\operatorname{dos}) / m_{n}^{*}(\operatorname{dos})=1.2$. The dashed, dotted and solid curves are valid for strictly extrinsic (very low temperatures) and strictly intrinsic (high temperatures). The solid curve presents the variation for the n-type system characterised with the above parameters. 


\subsection{Transport coefficients for extrinsic semiconductors}

The transport coefficients for an extrinsic semiconductor can be expressed analytically using the simplified band picture presented in the preceding sub-section. For n-type semiconductors, the electronic relaxation time can be written as

$$
\tau=\tau_{0}(E-E c)^{p}
$$

where $p$ takes vales $-\frac{1}{2}$ and $\frac{3}{2}$ for scattering from (acoustic) phonons and ionisedimpurities, respectively. The factor $\tau_{0}$ is material and scattering process dependent and may also depend on temperature $[2,35,41]$. We also express

$$
\begin{aligned}
& E=E_{c}+\frac{\hbar^{2} k^{2}}{2 m_{c}^{*}} \text { for bulk }(D=3), \\
& E=E_{c}+E_{1}^{n}+\frac{\hbar^{2} k_{\text {in-plane }}^{2}}{2 m_{c}^{*}} \text { for } D=2 .
\end{aligned}
$$

Corresponding expressions for p-type semiconductors can be easily written. With assumed isotropy of $\boldsymbol{k}$-space, the summation of $\boldsymbol{k}$ in Eqs. (17) - (19) can be replaced by $\sum_{\boldsymbol{k}}=\int g(E) \mathrm{d} E$, where $g(E)$ is the spin-degenerate density of states at energy $E$, and the following energy integration-by-parts result can be used [2]

$$
\int_{0}^{\infty} \mathrm{e}^{r} \frac{\partial \bar{f}}{\partial \epsilon} \mathrm{d} \epsilon=-r \int_{0}^{\infty} \mathrm{e}^{r-1} \bar{f} \mathrm{~d} \epsilon
$$

Assuming further that the only carrier scattering is from acoustic phonons, we can express (see [41] for $D=3$ and [42] for $D=2$ )

$$
\sigma= \begin{cases}\frac{2 N_{\mathrm{val}} e^{2} \hbar \varrho c_{L}^{2}}{3 \pi E_{\mathrm{def}}^{2} m_{c}^{*}} \mathcal{F}_{0}\left(\beta_{n}\right), & \text { for bulk }(\mathrm{D}=3) ; \\ \frac{4 N_{\mathrm{val}} e^{2} \hbar \varrho c_{L}^{2}}{3 \pi E_{\mathrm{def}}^{2} m_{c}^{*}} \mathcal{F}_{0}\left(\beta_{n}\right) & \text { for quantum wells, superlattices }(\mathrm{D}=2),\end{cases}
$$

where $N_{\text {val }}$ is the number of valleys, $c_{L}$ is the velocity of the longitudinal phonon mode in the long-wavelength limit, $E_{\text {def }}$ is the acoustic deformation potential, $m_{c}^{*}$ is the conduction band effective mass and $\mathcal{F}_{0}\left(\beta_{n}\right)$ is defined in Eq. (27).

The Seebeck coefficient, for electrons, can be expressed as $[22,43]$

$$
S=-\frac{k_{\mathrm{B}}}{e}\left[\frac{(p+D / 2+1) \mathcal{F}_{p+D / 2}\left(\beta_{n}\right)}{(p+D / 2) \mathcal{F}_{p+D / 2-1}\left(\beta_{n}\right)}-\beta_{n}\right] .
$$

The carrier contribution to the thermal conductivity can be expressed as

$$
\kappa_{\text {carrier }}=T \sigma\left(\beta_{n}\right) \mathcal{L}\left(\beta_{n}\right)
$$

where $\mathcal{L}$ is the Lorentz number given as

$$
\mathcal{L}=\left(\frac{k_{\mathrm{B}}}{e}\right)^{2}\left[\frac{(p+D / 2+2) \mathcal{F}_{p+D / 2+1}\left(\beta_{n}\right)}{(p+D / 2) \mathcal{F}_{p+D / 2-1}\left(\beta_{n}\right)}-\left(\frac{(p+D / 2+1) \mathcal{F}_{p+D / 2}\left(\beta_{n}\right)}{(p+D / 2) \mathcal{F}_{p+D / 2-1}\left(\beta_{n}\right)}\right)^{2}\right]
$$

Sometimes it is convenient $[41,44]$ to express the conductivity in terms of the mobility $\mu$. This is achieved by expressing $\sigma=n|\mathcal{Q}| \mu$, with $\mu=\frac{|\mathcal{Q}|}{m_{c}}<\tau>$, where $<\tau>$ is the energy average of the relaxation time defined as

$$
<\tau>=\frac{\int_{0}^{\infty} \tau(E) E^{3 / 2} \frac{\partial \bar{f}}{\partial E} \mathrm{~d} E}{\int_{0}^{\infty} E^{3 / 2} \frac{\partial \bar{f}}{\partial E} \mathrm{~d} E} \approx \frac{\int_{0}^{\infty} \tau(W) W^{3 / 2} \exp (-W) \mathrm{d} W}{\int_{0}^{\infty} W^{3 / 2} \exp (-W) \mathrm{d} W} ; \quad W=E / k_{B} T
$$


Using $\langle\tau>$ rather than $\tau(E)$ in Eqs. (17)-(19), or Eqs. (20)-(22), has been referred to as the constant relaxation time approach (CRTA), which assumes that the carrier scattering rate is independent of its energy.

It is useful here to present expressions for the electronic transport coefficients using the constant relaxation time approximation (CRTA) and parabolic bands. For conduction along the $x$ direction, Hicks and Dresshaus obtained the following expressions:

(i) $3 \mathrm{D}$ n-type bulk material, with the dispersion relation $E\left(k_{x}, k_{y}, k_{z}\right)=\frac{\hbar^{2}}{2}\left(\frac{k_{x}^{2}}{m_{x}^{*}}+\right.$ $\left.\frac{k_{y}^{2}}{m_{y}^{*}}+\frac{k_{z}^{2}}{m_{z}^{*}}\right)[44]$ :

$$
\begin{aligned}
S^{\mathrm{CRTA}} & =-\frac{k_{\mathrm{B}}}{e}\left(\frac{5 \mathcal{F}_{3 / 2}}{3 \mathcal{F}_{1 / 2}}-\zeta\right), \\
\sigma^{\mathrm{CRTA}} & =\frac{e \mu_{x}}{2 \pi^{2}}\left(\frac{2 k_{\mathrm{B}} T}{\hbar^{2}}\right)^{3 / 2}\left(m_{x}^{*} m_{y}^{*} m_{z}^{*}\right)^{1 / 2} \mathcal{F}_{1 / 2}, \\
\kappa_{\text {carriers }}^{\mathrm{CRTA}} & =\frac{<\tau>k_{\mathrm{B}} \hbar^{2}}{6 \pi^{2}}\left(\frac{2 k_{\mathrm{B}} T}{\hbar^{2}}\right)^{5 / 2}\left(\frac{m_{y}^{*} m_{z}^{*}}{m_{x}^{*}}\right)^{1 / 2}\left(\frac{7}{2} \mathcal{F}_{5 / 2}-\frac{25 \mathcal{F}_{3 / 2}^{2}}{6 \mathcal{F}_{1 / 2}}\right),
\end{aligned}
$$

where $m_{x}^{*}, m_{y}^{*}, m_{z}^{*}$ are the effective-mass components and $\mu_{x}$ is the (constant) mobility in the $x$ direction.

(ii) 2D n-type material of square cross section, with a side of length $d_{A}$ and dispersion relation $E\left(k_{x}, k_{y}\right)=\frac{\hbar^{2}}{2}\left(\frac{k_{x}^{2}}{m_{x}^{*}}+\frac{k_{y}^{2}}{m_{y}^{*}}\right)+\frac{\hbar^{2} \pi^{2}}{2 m_{z}^{*} d_{A}^{2}}[44]$ :

$$
\begin{aligned}
S^{\mathrm{CRTA}} & =-\frac{k_{\mathrm{B}}}{e}\left(\frac{2 \mathcal{F}_{1}}{\mathcal{F}_{0}}-\beta_{n}\right), \\
\sigma^{\mathrm{CRTA}} & =\frac{e \mu_{x}}{2 \pi d_{A}}\left(\frac{2 k_{\mathrm{B}} T}{\hbar^{2}}\right)\left(m_{x}^{*} m_{y}^{*}\right)^{1 / 2} \mathcal{F}_{0}, \\
\kappa_{\text {carriers }}^{\text {CRTA }} & =\frac{<\tau>k_{\mathrm{B}} \hbar^{2}}{4 \pi d_{A}}\left(\frac{2 k_{\mathrm{B}} T}{\hbar^{2}}\right)^{2}\left(\frac{m_{y}^{*}}{m_{x}^{*}}\right)^{1 / 2}\left(3 \mathcal{F}_{2}-\frac{4 \mathcal{F}_{1}^{2}}{\mathcal{F}_{0}}\right) .
\end{aligned}
$$

(iii) 1D n-type material of square cross section, with a side of length $d_{A}$ and dispersion relation $E\left(k_{x}\right)=\frac{\hbar^{2} k_{x}^{2}}{2 m_{x}^{*}}+\frac{\hbar^{2} \pi^{2}}{2 d^{2}}\left(\frac{1}{m_{y}^{*}}+\frac{1}{m_{z}^{*}}\right)[45]$ :

$$
\begin{aligned}
S^{\mathrm{CRTA}} & =-\frac{k_{\mathrm{B}}}{e}\left(\frac{3 \mathcal{F}_{1 / 2}}{\mathcal{F}_{-1 / 2}}-\beta_{n}\right), \\
\sigma^{\mathrm{CRTA}} & =\frac{e \mu_{x}}{\pi d_{A}^{2}}\left(\frac{2 k_{\mathrm{B}} T}{\hbar^{2}}\right)^{1 / 2}\left(m_{x}^{*}\right)^{1 / 2} \mathcal{F}_{-1 / 2}, \\
\kappa_{\text {carriers }}^{\mathrm{CRTA}} & =\frac{<\tau>k_{\mathrm{B}} \hbar^{2}}{\pi d_{A}^{2}}\left(\frac{2 k_{\mathrm{B}} T}{\hbar^{2}}\right)^{3 / 2}\left(\frac{1}{m_{x}^{*}}\right)^{1 / 2}\left(\frac{5}{2} \mathcal{F}_{3 / 2}-\frac{9 \mathcal{F}_{1 / 2}^{2}}{2 \mathcal{F}_{-1 / 2}}\right) .
\end{aligned}
$$

The expressions in Eqs. (43)-(45) have been routinely employed in numerical calculations of the transport coefficients. However, the assumption underlying the CRTA that the carrier scattering time $\langle\tau\rangle$, or the mobilty $\mu_{x}$, is independent of carrier energy and dimension(s) of a system is unrealistic and may produce physically unappealing results, as has been emphasized by Broido and Reinecke [46], and it is advisable to use the full theoretical treatment of the carrier transport coefficient described earlier. 


\subsection{Transport Coefficients for intrinsic semiconductors}

The nature of a doped semiconductor turns from extrinsic to intrinsic at high temperatures when the dopant carriers are fully ionised and thermally excited host electrons and holes begin to contribute to the transport properties. The transport coefficients are then expressed as $[2,47]$

$$
\begin{aligned}
\sigma_{\text {tot }} & =\sigma_{n}+\sigma_{p}, \\
S_{\text {tot }} & =\frac{\sigma_{n} S_{n}+\sigma_{p} S_{p}}{\sigma_{\text {tot }}}, \\
\kappa_{\text {carriers }} & =\left(\frac{k_{\mathrm{B}}}{e}\right)^{2} T\left\{\mathcal{L}\left(\beta_{n}\right) \sigma_{n}+\mathcal{L}\left(\beta_{p}\right) \sigma_{p}+\frac{\sigma_{n} \sigma_{p}}{\left(\sigma_{n}+\sigma_{p}\right)}\left[r_{1}+r_{2}+\zeta_{g}\right]^{2}\right\},
\end{aligned}
$$

where $r_{1}$ and $r_{2}$ are dimensionless positive parameters depending on the electron and hole scattering processes, and $\zeta_{g}$ is the reduced band gap. Assuming predominance of scattering from phonons, it is estimated that $r_{1}=r_{2}=2$. In the above equation, the first two terms represent monopolar contributions (from electrons and holes, respectively) and the thrid term is the bipolar contribution $\left(\kappa_{\mathrm{bp}}\right)$ from electron-hole pairs.

Consider an n-type bulk semiconductor $(D=3)$ within a single band approximation. At temperatures when intrinsic behaviour becomes important the band edges are much farther apart than the thermal energy $k_{\mathrm{B}} T$, so that the Fermi-Dirac distibution function for excited electrons in the conduction band and excited holes in the valence band can be expressed in the Botzmann form $\bar{f}_{e}=\exp \left[\left(E_{F}-E c\right) / k_{\mathrm{B}} T\right]$ and $\bar{f}_{h}=\exp \left[\left(E_{v}-E_{F}\right) / k_{\mathrm{B}} T\right]$, respectively. With this consideration the Fermi integral in Eq. (27) can be expressed as

$$
\mathcal{F}_{n}^{\text {classical }}(y)=\mathrm{e}^{y} \Gamma(n+1)
$$

Equation (24) then is heavily modified and the electron and hole concentrations are given by [39]

$$
n_{i}=p_{i}=2\left(\frac{k_{\mathrm{B}} T}{2 \pi \hbar^{2}}\right)^{3 / 2}\left(m_{n}^{*} m_{p}^{*}\right)^{3 / 4} \exp \left(-\zeta_{g} / 2\right)
$$

The following expressions can then be easily derived:

$$
\begin{aligned}
\sigma_{\text {intrinsic }}^{\mathrm{CRTA}} & =\frac{\sqrt{\pi} e}{4 \pi^{2}}\left(\frac{2 k_{\mathrm{B}} T}{\hbar^{2}}\right)^{3 / 2}\left(m_{n}^{*} m_{p}^{*}\right)^{3 / 4}\left(\mu_{n}+\mu_{p}\right) \exp \left(-\zeta_{g} / 2\right) \\
S_{\text {intrinsic }} & =-\frac{k_{\mathrm{B}}}{e}[(p+5 / 2)-\zeta] \\
\mathcal{L}_{\text {intrinsic }} & =(p+5 / 2) .
\end{aligned}
$$

When using these expressions, it should be remembered that as both $\tau$ and $m^{*}$ usually vary with temperature, so does the mobility. The temperature dependence of the mobility $\mu$ is material dependent, but is expected to be somewhat different from $T^{-3 / 2}$.

\section{Phonon transport coeffient}

Having described the carrier transport coefficients in a resonable manner, we attempt now to discuss in some detail several issues related to the phonon transport coefficient, 
viz. the lattice thermal conductivity (phonon conductivity) of bulk and low-dimensional semiconductors. Of particular importance will be the question of how to tune phonon properties with a view to seeking enhancement in the TE figure of merit $Z T$. In order to achieve our aim, we would need first to gain knowledge of phonon modes, then develop a suitable theory of phonon transport, and finally examine some of the factors that control lattice thermal conductivity of low-dimensional systems, particularly in the form of nanocomposites.

\subsection{Phonons in bulk thermoelectric materials}

4.1.1. Lattice dynamical problem: Determination of phonon eigenvalues (i.e. frequencies) and eigenvectors (i.e. atomic displacement pattern) at desired wavevectors inside the Brillouin zone of a crystalline structure is generally known as the lattice dynamical study. This has traditionally been attempted at two levels: at the atomic level using the concept of inter-atomic harmonic force constants, and at the continuum level treating a solid as a continuum and using the concept of second-order elastic constants. Atomic level theories have been based on either phenomenological models (see, [48, 49] for details) or at first-principles level (see, e.g. [49, 50]). First-principles approaches (based on the application of Density Functional Theory for electron-electron interactions and perturbation theory (DFPT) [50]) present the state-of-the art but, depending on local computational facilities, may not be affordable for crystal structures with very large unit cell volumes. Physically appealing phenomenological approaches such as the adiabatic bond charge model (BCM) [51], and (semi)empirical approaches based on applications of interatomic potentials [52, 53, 54, 55, 56, 59], can be applied to relatively larger unit cells of tetrahedrally bonded systems. For much larger unit cells, and thus for nanostructures of physically realisable sizes, elastic and dielectric continuum models of phonons become a necessity. Such models rely on good estimates of elastic constants and dielectric constants required for the crystal symmetry under study [60].

The following procedure can be adopted to set up the lattice dynamical problem at the atomic level. Let $\boldsymbol{x}(l b)=\boldsymbol{x}(l)+\boldsymbol{x}(b)$ denote the position of $b^{\text {th }}$ atom of mass $M_{b}$ in $l^{\text {th }}$ unit cell of a periodic system. A trial function for atomic vibrations can be expressed as

$$
u_{\alpha}(l b)=\frac{1}{\sqrt{M_{b}}} \sum_{\boldsymbol{q}} U_{\alpha}^{\prime}(\boldsymbol{q} ; b) \exp [i(\boldsymbol{q} \cdot \boldsymbol{x}(l b)-\omega t)],
$$

with $U$ being the vibrational amplitude. Solutions to the lattice dynamical problem can be obtained by solving the following eigenvalue equations

$$
\omega^{2} e_{\alpha}(b ; \boldsymbol{q} s)=\sum_{b^{\prime} \beta} C_{\alpha \beta}\left(b b^{\prime} \mid \boldsymbol{q}\right) e_{\beta}\left(b^{\prime} ; \boldsymbol{q} s\right) .
$$

Here $\boldsymbol{e}(b ; \boldsymbol{q} s)$ is the eigenvector of atomic displacements and $C_{\alpha \beta}\left(b b^{\prime} \mid \boldsymbol{q}\right)$ is a Fourier 
component of the inter-atomic force constant matrix $\Phi_{\alpha \beta}\left(0 b ; l b^{\prime}\right)$ :

$$
C_{\alpha \beta}\left(b b^{\prime} \mid \boldsymbol{q}\right)=\frac{1}{\sqrt{M_{b} M_{b^{\prime}}}} \sum_{l} \Phi_{\alpha \beta}\left(0 b ; l b^{\prime}\right) \exp \left[-i \boldsymbol{q} \cdot\left(\boldsymbol{x}(0 b)-\boldsymbol{x}\left(l b^{\prime}\right)\right)\right] .
$$

The force constant matrix $\left\{\Phi_{\alpha \beta}\left(l b ; l^{\prime} b^{\prime}\right)\right\}$ is obtained from the harmonic part of the total crystal potential energy. This is achieved $a b$ inito in first-principles methods and by means of adjustable parameters in phenomenological methods. For $N_{b}$ basis atoms in a unit cell, there will be 3 acoustic branches (two transverse, or TA, and one longitudinal, or LA) and $3\left(N_{b}-1\right)$ optical branches.

Phenomenological approaches to lattice dynamical studies have a long history, the first attempt being made a hundred years ago. Such studies can be broadly classified in three categories: (i) force constant models, (ii) rigid ion models and (iii) dipole models. Examples of force constant models include the Born model [61], the Born-von Kàrman model (see [62]) and the valence force field model [63, 64, 65]. The rigid ion model has been applied to ionic solids [66] as well as to tetrahedrally bonded semiconductors $[67,68]$. Examples of dipole models include the shell model [69, 70, 71], the deformable (or breathing) shell model $[72,73]$ and the bond charge model $[65,74]$. The bond charge model was developed into an adiabatic bond charge model (BCM) for successful lattice dynamical studies of tetrahedrally bonded semiconductors $[51,75,76]$. Since the 1980s there have been numerous successful applications of the (semi)empirical approaches based on applications of interatomic potentials for calculations of phonon dispersion relations in tetrahedrally-bonded semiconductors (see, e.g. $[52,53,54,55,56,59]$ ).

We will briefly describe the essence of the adiabatic bond charge model (BCM) and the first-principles method (DFPT), and present results of calculations for a few bulk and nanostructured TE materials.

\section{(i) Adiabatic bond charge model:}

In covalently bonded semiconductors (e.g. Si and $\mathrm{Ge}$ ) and in partially covalently bonded semiconductors (e.g. III-V's and II-VI's) the majority of the valence charge is located along bonds between nearest neighbours. The bond charge model $[51,75,76]$ treats such systems as a collection of ionic charges at atomic sites and bond charges at the locations of valence charge maxima along inter-atomic bonds. This concept is illustrated in Fig. 2 for the zincblende structure. In the adiabatic bond charge model, a bond charge (bc) is assigned zero mass and its motion adiabatically follows that of ions. Three types of inter-particle interactions are considered: (a) ion-ion, ion-bc, bc-bc Coulomb interaction, (b) ion-ion and ion-bc central short-range interaction (for heteropolar semiconductors bc-bc short-range interaction is also considered) and (c) ion-bc and bc-bc non-central (or bond-bending) interaction of Keating type [64]. The total number of disposable BCM parameters is four for diamond structure purely covalent materials (e.g. diamond, Si, Ge) and six for zincblende structure partly covalent materials (e.g. GaAs, CdTe, etc.). A detailed discussion of how the dynamical matrix is set up can be found in Ref. [49]. 

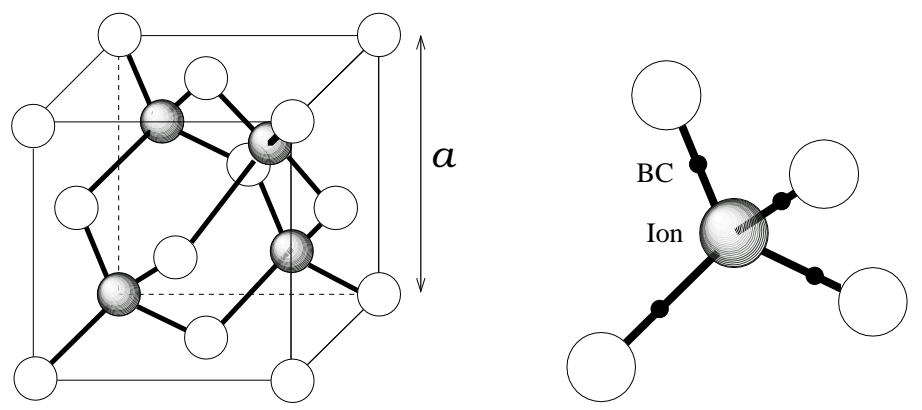

Figure 2. Illustration of the BCM concept for the arrangement of ions (open and filled spheres) and bond charges (small filled spheres) with tetrahedral bonding between nearest neighbour atoms in the zincblende structure.

(ii) First-principles Aapproaches:

Several levels of first-principles approaches have been adopted for phonon calculations [49], such as the frozen phonon method, the restricted dynamical matrix method, the planar force constant method and a perturbative method within the linear response approach. While other methods are applied to determine phonon modes at symmetry points or along symmetry directions inside the Brillouin zone of the system under consideration, the last approach can be applied for calculations of phonon eigen solutions throughout the Brillouin zone. The method uses a combination of the density functional and perturbation theories (hence known as DFPT) and can in principle be applied with any $a b$ initio method of electronic band structure calculations (such as the tight-binding method, the full-potential augmented plane wave method, atomic-orbitalbased pseudopotential method and the planewave pseudopotential method). The most common of these is the planewave pseudopotential method, which has been discussed in detail by Giannozzi et al. [77]. Here we point out the essential steps of the DFPT approach.

For a given atomic displacement $\boldsymbol{u}$ the electronic part of the total energy is expressed as

$$
E_{\mathrm{el}}(\boldsymbol{u})=E_{\mathrm{el}}(\mathbf{0})+\delta E_{\mathrm{el}}\left(\boldsymbol{u}, \Delta \rho_{\mathrm{el}}\right),
$$

where $\Delta \rho_{\mathrm{el}}$ is the resulting change in the electronic charge density. The electronic contribution to the force constant matrix $\Phi$ depends on $\Delta \rho_{\mathrm{el}}$ and the change $\Delta V_{\mathrm{KS}}$ in the Kohn-Sham potential. A self-consistent change in $\Delta V_{\mathrm{KS}}$ is expressed up to terms linear in $\Delta \rho_{\mathrm{el}}$. Similarly, using first-order perturbation theory, $\Delta \rho$ is expressed in terms of $\Delta V_{\mathrm{KS}}$. An iterative solution is obtained for $\Delta \rho_{\mathrm{el}}$ and $\Delta V_{\mathrm{KS}}$ by solving the two relationships.

(iii) Continuum approximation:

In the long wavelength limit phonon dispersion relations can be derived by treating a crystalline structure as a continuum with an equivalent mass density. Acoustic and optical phonon phenomena can be discussed by adopting the elastic continuum and 
dielectric continuum models, respectively [60]. Analysis of many thermal properties have been made by considering only the long wavelength acoustic phonon branches. For isotropic media, this results in the linear dispersion relation $\omega(\boldsymbol{q} s)=\omega(q, s)=c_{s} q$, where $s=\mathrm{TA}(\mathrm{LA})$ indicates the transverse(longitudinal) polarisation branch. The concept of Brillouin zone is replaced with that of the Debye sphere of radius $q_{\mathrm{D}}$ for an isotropic $3 \mathrm{D}$ continuum, ensuring that its volume accommodates all acoustic phonon modes [48].

4.1.2. Phonon density of states: With phonon frequencies $\omega(\boldsymbol{q} s)$ available for modes $\boldsymbol{q} s$ throughout the Brillouin zone for the crystal structure under consideration, the density of phonon states (DOS) at frequency $\omega$ can be evaluated using the relation

$$
g(\omega)=\sum_{\boldsymbol{q}_{s}} \delta(\omega-\omega(\boldsymbol{q} s))
$$

Several methods have been proposed and employed for performing Brillouin zone summations of the type required for performing DOS and related calculations. Let us consider the summation of a general periodic function $f(\boldsymbol{q})$

$$
I=\sum_{\boldsymbol{q}} f(\omega(\boldsymbol{q}))
$$

If the function $f(\omega(\boldsymbol{q}))$ is isotropic with a linear dispersion relation, i.e. $\omega(\boldsymbol{q})=c q$, then the summation can be performed by using the Debye scheme. In this scheme the Brillouin zone summation for a cubic isotropic function is expressed as an integral over the Debye sphere of radius $q_{\mathrm{D}}$

$$
I=\int_{0}^{\omega_{D}} g_{D}(\omega) f(\omega) \mathrm{d} \omega
$$

where

$$
g_{D}(\omega)=\frac{N_{0} \Omega}{2 \pi^{2}} \frac{\omega^{2}}{c^{3}}
$$

is the Debye density of states function and $\omega_{D}=c q_{\mathrm{D}}$ is the Debye frequency. The simple isotropic continuum Debye method must be improved adequately to deal with non-cubic crystal structures, and dispersive phonon modes in acoustic as well as optical branches.

For more general situations, efficient and accurate numerical evaluation of the Brillouin zone summation can be made by considering a set of 'special $\boldsymbol{q}$-points', constructed by following a technique such as that described by Monkhorst and Pack [78]. For a choice of $N_{\mathrm{sp}}$ special $\left\{\boldsymbol{q}_{i}\right\}$ points inside the central Brillouin zone and weight factors $\left\{W\left(\boldsymbol{q}_{i}\right)\right\}$ associated with them, the integral $I$ can be estimated as

$$
I \simeq N_{0} \sum_{i}^{N_{\mathrm{sp}}} f\left(\omega\left(\boldsymbol{q}_{i}\right)\right) W\left(\boldsymbol{q}_{i}\right),
$$

where $N_{0}$ is the number of unit cells in the crystal. For a given shape of Brillouin zone, different sets of special $\left\{\boldsymbol{q}_{i}\right\}$ points can be generated. A set is considered more 'efficient' if it provides an acceptable result for the integral with the least number of $\left\{\boldsymbol{q}_{i}\right\}$ points [79]. 
Using the 'special $\boldsymbol{q}$-points' method, the expression in Eq. (58) for DOS can be evaluated as

$$
g(\omega) \simeq N_{0} \sum_{i}^{N_{s p}} W\left(\boldsymbol{q}_{i}\right) \delta\left(\omega-\omega\left(\boldsymbol{q}_{i} s\right)\right) .
$$

The Dirac delta function in the above equation can be replaced by a Gaussian function of width $\xi$, allowing evaluation of the DOS in the form

$$
g(\omega)=\lim _{\xi->0} \frac{N_{0}}{\xi \sqrt{\pi}} \sum_{i}^{N_{s p}} W\left(\boldsymbol{q}_{i}\right) \exp \left[-\left(\omega-\omega\left(\boldsymbol{q}_{i} s\right)\right)^{2} / \xi^{2}\right] .
$$

4.1.3. Results for bulk Si and Ge: The phonon dispersion curves for Si and Ge, obtained theoretically using the BCM and measured experimentally using neutron scattering experiments, are shown in Fig. 3 along several symmetry directions in the fcc Brillouin zone. With 2 basis atoms per fcc lattice point for the diamond structure, there are in all six phonon branches. Close to the zone centre (the $\Gamma$ point), in increasing order of frequency these branches are: two TA, one LA, two transverse optical TO and one longitudinal optical LO. It can be noted that close to $\Gamma$ the dispersion is linear, though not isotropic. The maximum acoustic and optical frequencies, respectively, are close to $11 \mathrm{THz}$ and $15.8 \mathrm{THz}$ in $\mathrm{Si}$, and close to $7 \mathrm{THz}$ and $9.5 \mathrm{THz}$ in Ge. There is no acoustic-optical gap for either bulk.
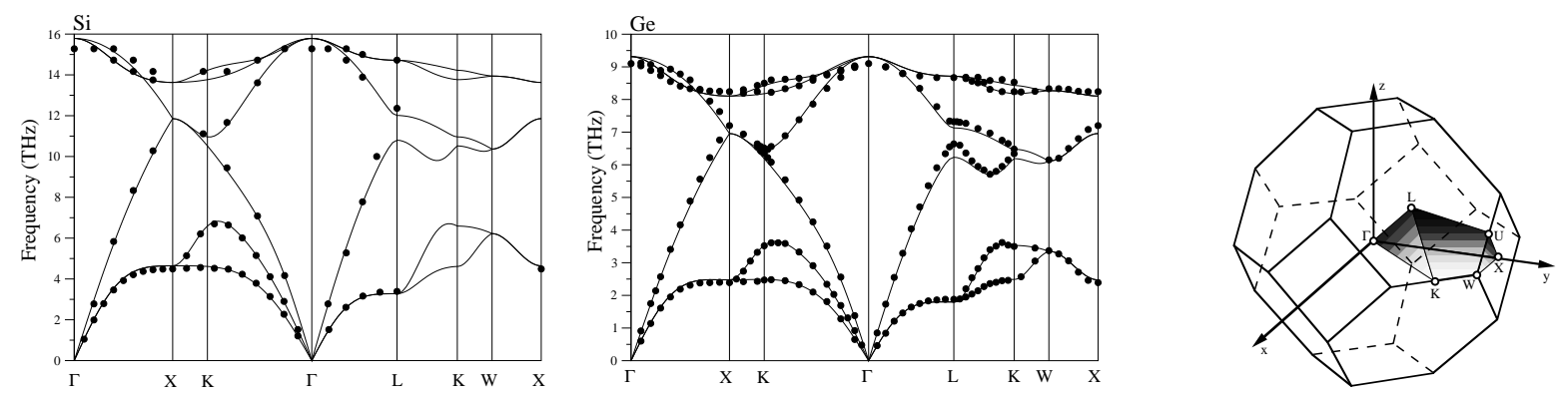

Figure 3. Phonon dispersion curves for Si and Ge. Solid curves are obtained from the application of the adiabatic bond charge model (taken from Ref. [80]) and the symbols represent measurements made from neutron scattering experiments (taken from Refs. $[81,82,83,84])$. Also shown are various symmetry points in the fcc Brillouin zone.

Figure 4 presents the DOS for bulk Si, evaluated numerically from the application of the BCM. There are peaks (indicating van Hove singularities) at frequencies approximately equal to 4, 7.6, 8.4 and $12.5 \mathrm{THz}$. For comparison, the DOS of the corresponding elastic continuum, with an average acoustic phonon speed of $5691 \mathrm{~m} / \mathrm{s}$, is also plotted on the same graph. Clearly, the DOS of the continuum matches well with the realistic numerical results only in the low frequency range 0-3.5 THz. Apart from significant departure from the realistic results for high frequencies, the continuum theory indicates a van Hove singularity at around $9.5 \mathrm{THz}$, close to a dip in the realistic curve. 


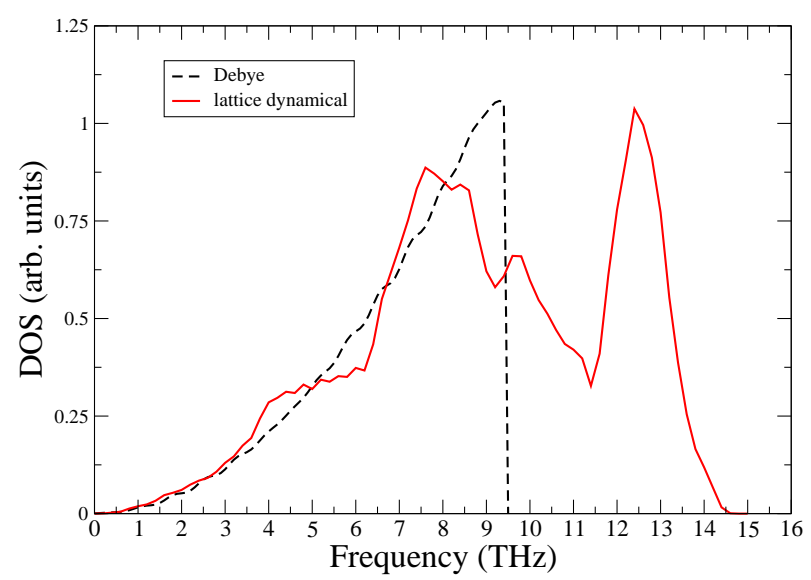

Figure 4. Phonon density of states for bulk Si. The curves with solid and dotted lines represent results from the application of the BCM model and for Debye's isotropic continuum model, respectively.

\subsection{Phonons in nanostructured thermoelectric materials}

As explained by Chen et al. [18] there are two schools of thought for developing theoretical explanations for the thermal conductivity of nanostructures, such as superlattices. In one approach, one develops a theory based on calculations of the phonon spectrum, phonon velocity, and phonon density of states. In another approach, one treats phonons are treated as particles and their reflection and transmission across an interfaces is treated classically. In the first approach, phonons are totally coherent, as long as the interfaces and internal scattering do not destroy the coherence of the phonons. It should be borne in mind that the discussion in this review article is based on using both the approaches.

A nanostructured material, such as a nanowire, a nanodot or a quantum well, can be modelled for phonon computation using one of the methods described in the preceding sub-section by adopting an artificially repeated structure, with a large unit cell (LUC) containing the nanostructure and an adequate vacuum region to avoid interaction between neighbouring nanostructures [49]. No vacuum region is required when modelling a superlattice, and the LUC is defined by the repeat period of the structure. The crystal symmetry of such systems will be lower than that of bulk systems. A nanocomposite contains at least two materials (of different masses) in each of its LUC. This essentially gives rise to the gap opening both at the centre and edges of the Brillouin zone of the newly constructed periodic structure. Presence of surface(s) and formation of interfaces can, respectively, give rise to surface and interface phonon modes. DFT-based firstprinciples methods can be straightforwardly applied to such structures. However, when applying a phenomenological method, such as the BCM, an adjustment must be made to deal with hetero bonds at the interfaces between different material species within each LUC. 
Phonon dispersion relations of nanostructured materials are characterised as having size-dependent and symmetry-reduction features. In addition, there can be compositiondependent features for nanocomposites. Thus, substantial changes in phonon dispersion relations can be expected, or achieved, by nanostructuring. In general, three types of new features can be expected: branch foldings, gap openings and mode confinements. Each phonon phonon branch in the bulk zone can be plotted as a few branches in the new (smaller) Brillouin zone for the nanostructure. This is done by 'folding' the bulk branch at the edge and the centre of the zone for the nanostructure. If, for example, the structural periodicity in a nanostructure is twice that of the corresponding bulk structure, then a phonon dispersion curve is folded once at the nanostructure zone boundary. If the composition of a nanostructure contains more than one material, then in general the folding (at the zone edge, or the zone centre) may be accompanied by splitting of the otherwise degenerate branches. Such splittings are known as gap openings. The folding and splitting features are accompanied by flatness in the splitted branches. Some of the higher-lying phonon branches tend to be characterised by very low dispersion, e.g. the corresponding modes become confined. Raman scattering studies [57] have confirmed the confinement of optical branches in semiconductor superlattices. Theoretical studies [58] have indicated that higher frequency acoustic phonons in superlattices are also characterised by confinement. In fact such modes are more like standing waves within each layer of a superlattice, polarized normally to the interfaces of the superlattice, and therefore are non-propagating.

In order to make the BCM calculations for nanowires, nanoslabs and nanodots, Hepplestone and Srivastava [85] employed the above mentioned concept of a periodic supercell, with each LUC containing the nanostructure and an adequate vacuum region around it. They set the effective mass of atoms present in the vacuum region as $10^{-6}$ that of a single Si atom.

\section{Si nanowires:}

Phonon dispersion relations of free standing Si nanowires have been obtained by several groups employing different techniques. These include the works of Mingo and Yang [59], Thonhauser and Mahan [86], Nishiguchi et al. [87], and Li et al. [88]. Mingo and Yang [59] employed Harrison's interatomic potential that uses only two- and three-body terms. For a free standing nanowire four acoustic branches are obtained: one dilatational, one torsional (or shear) and two flexural. While the dilatational and tortional branches have a linear disperion relation, the two flexural branches have a quadratic dispersion relation.

The embedding technique employed by Hepplestone and Srivastava [85] for nanowires almost mimics the clamped boundary conditions employed by Thonhauser and Mahan [86] and has the advantage that the resulting phonon dispersion curves represent those of a nanostructure on a substrate. However, the disadvantage of this technique is that, as the rotational freedom is not available, it would not allow for the fourth acoustic branch obtained for a free standing nanowire [87]. 
The phonon dispersion curves and density of states obtained by Hepplestone and Srivastava [85] for an ultrathin Si nanowire (NW) of cross-section $0.543 \mathrm{~nm} \times 0.543 \mathrm{~nm}$ and growth direction [001], obtained from BCM calculations [85], are shown in Fig. 5. The size of the Brillouin zone along the growth direction, $\Gamma-Z$ is half of the distance $\Gamma$-X for the bulk zone in Fig. 3. A close comparison of the dispersion curves along $\Gamma-Z$ for the NW with those along $\Gamma$-X for the bulk clearly suggests that both folding and gap openings have taken place upon the wire formation. The acoustic modes have very high group velocities near the zone centre in the propagation direction $(\Gamma-Z)$, and all non-acoustic modes are almost flat and dispersionless in all directions. $\Gamma-\mathrm{X}$ and $\Gamma-\mathrm{K}$ are confinement directions, and the degree of flatness of the optical branches along these directions shows the lack of interaction between neighbouring nanowires in the supercell geometry adopted for the modelling of a single nanowire. There are several gaps in the DOS, located both within and above the bulk Si acoustic range. Also, the DOS peaks are sharp, with delta-like very narrow widths.

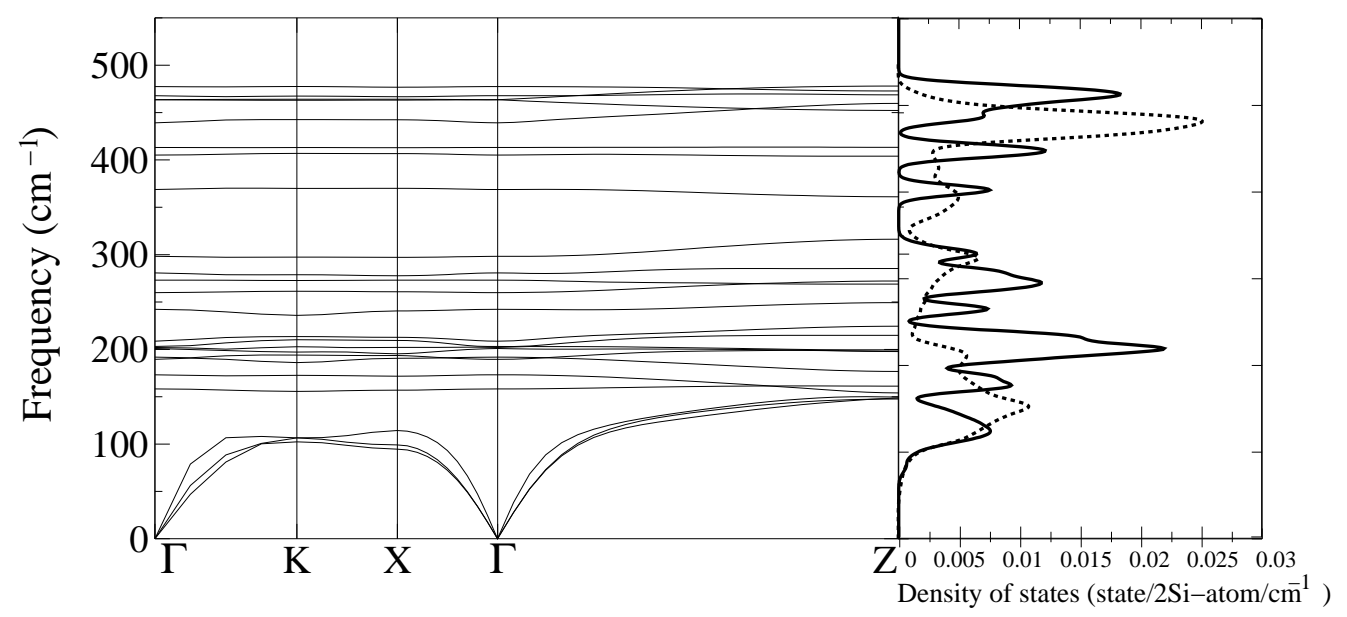

Figure 5. Phonon dispersion curves and density of states for a Si nanowire of crosssection $0.543 \mathrm{~nm} \times 0.543 \mathrm{~nm}$ and infinite length along [001]. $\Gamma$-X and $\Gamma-Z$ represent directions perpendicular and along the wire growth, respectively, and $\Gamma-\mathrm{K}$ is along the face diagonal in the Brillouin zone for the structure. The DOS curves for the wire and bulk are shown with bold and dashed lines, respectively. Taken with permission from [85].

As the wire thickness increases, the gaps in the DOS decrease rapidly. However, in the lower acoustic range the DOS increases in flat steps. This is in strong agreement with the prediction of the Debye continuum model that $\omega \propto q$ and $g(\omega) \propto \omega^{0}$. The energy of the highest optical mode is shifted downwards due to localisation. The position of the highest-lying peak in the DOS shifts gradually upwards with wire thickness, tending towards the bulk peak for thick wires. The group velocity of the acoustic modes decreases with increase in wire thickness, dropping below the corresponding bulk value. This behaviour of the group velocity, obtained from the full three-dimensional lattice 
dynamical calculations, is different from the results obtained from the elastic continuum model [60] which predicts a constant group velocity that is much smaller than the bulk value regardless of thickness. However, extrapolation of results of full calculations suggest that with increases in wire thickness, the group velocity will decrease towards the results obtained from the continuum theory.

\section{Si nanoslabs and nanodots:}

Figure 6 shows BCM results for phonon dispersion curves for a Si nanoslab of thickness $0.543 \mathrm{~nm}$ (left panel) and nanodot of size $0.53 \mathrm{~nm} \times 0.543 \mathrm{~nm} \times 0.543 \mathrm{~nm}$. In the nanoslab the phonon branches are flat and dispersionless in the direction of confinement, though not to the same degree as for the thinnest nanowire. The nanodot offers confinemnet in all three directions, as expected. As the system size increases, the nanodots show much greater flatness in dispersion curves than a nanowire of the same size of confinement. Similar to nanowires, the phonon DOS of nanoslabs and nanodots are characterised by the appearance of several gaps for the ultrathin size. The number and widths of gaps decrease rapidly as the size increases, as seen from the results presented in Fig. 7. A closer examination reveals that the DOS in the acoustic region follows the prediction of the Debye continuum model, i.e. $g(\omega) \propto \omega, g(\omega) \propto \omega^{0}$ and $g(\omega) \propto \omega^{-1}$ for the nanoslab, nanowire, and nanodot systems, respectively.

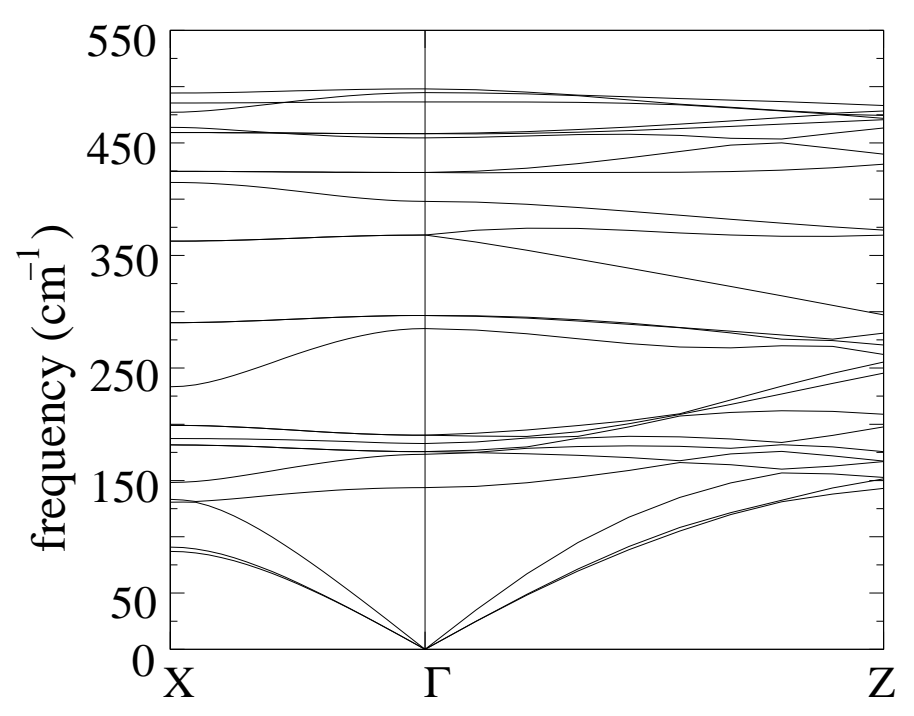

(a)

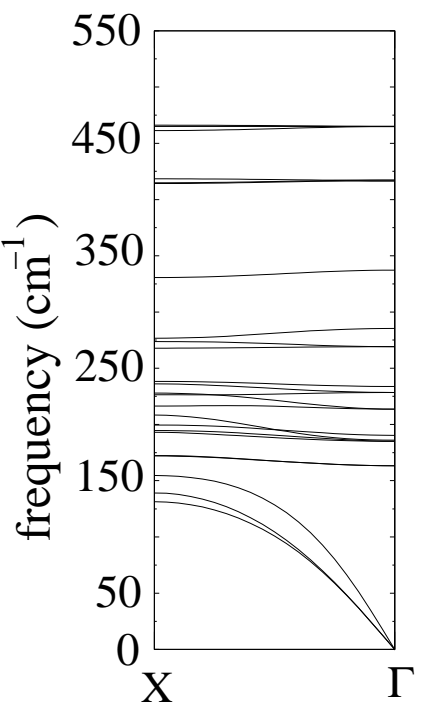

(b)

Figure 6. Phonon dispersion curves for (a) Si nanoslab on face (001) of thickness $0.543 \times 0.543 \mathrm{~nm}$, and (b) Si nanodot of size $0.543 \mathrm{~nm} \times 0.543 \mathrm{~nm} \times 0.543 \mathrm{~nm}$. Taken with permission from [85].

Si/Ge superlattices:

As an example of a nanocomposite thermoelectric material we consider 

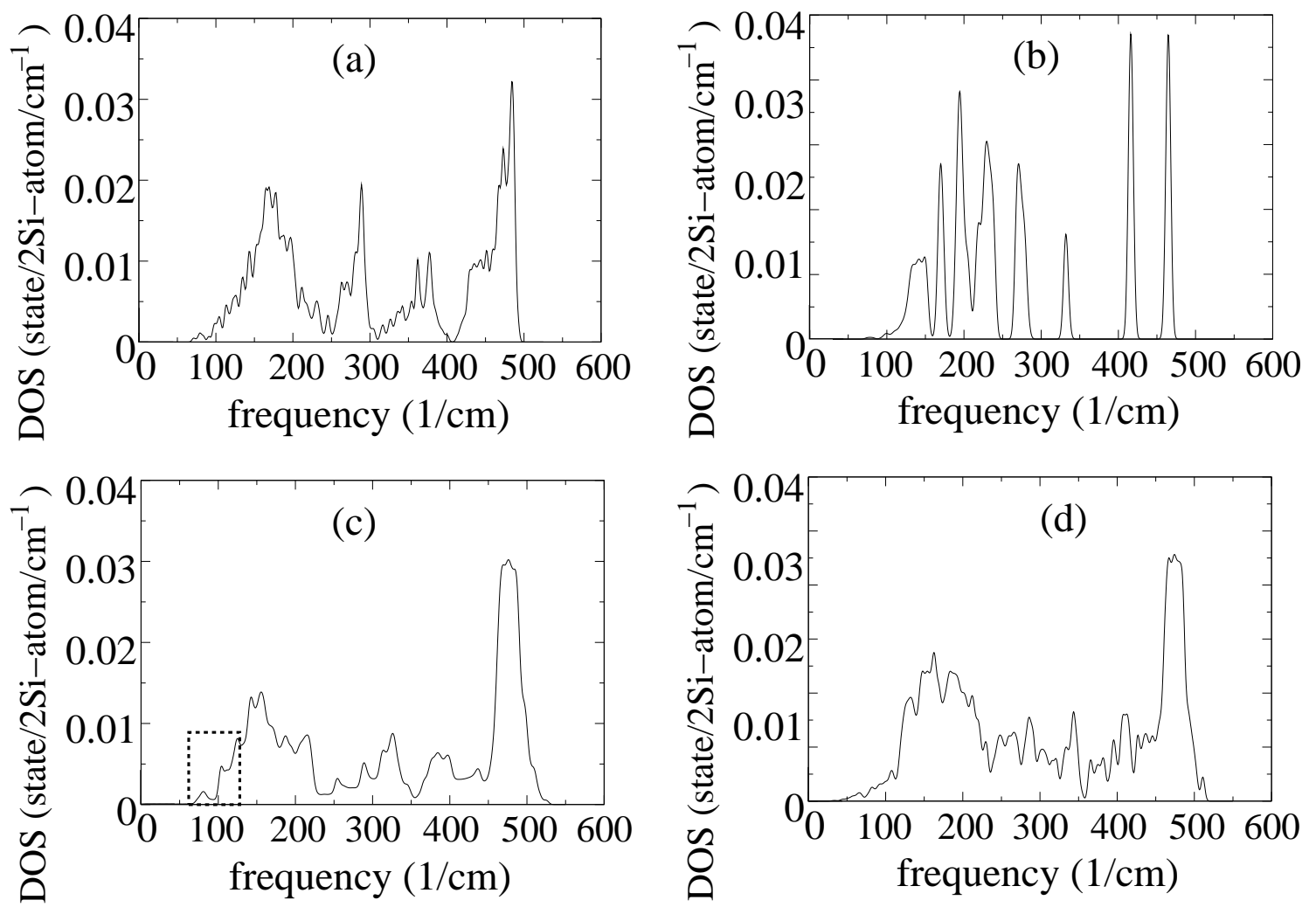

Figure 7. Density of phonon states for Si nanostructures: (a) nanoslab $0.543 \mathrm{~nm}$ thick, (b) nanodot of size $0.543 \mathrm{~nm} \times 0.543 \mathrm{~nm} \times 0.543 \mathrm{~nm}$, (c) nanoslab $32.520 \mathrm{~nm}$ thick, (d) nanodot of size $1.629 \mathrm{~nm} \times 1.629 \mathrm{~nm} \times 1.629 \mathrm{~nm}$. The continuum region is indicated by the box. Taken with permission from [85].

$\operatorname{Si}(n) \operatorname{Ge}(m)[001]$ superlattices, where $n$ and $m$ indicate the number of (001) bi-layers of Si and Ge atoms within a unit cell. For brevity we will denote these superlattice (SL) structures as $\mathrm{SL}(n, m)$. Detailed first-principles calculations of phonon dispersion curves for ultrathin $\operatorname{SL}(n, n)$ have been reported in Refs. [89, 90]. Here we make a few general observations.

The periodicity along the growth direction of $\operatorname{SL}(n, n)$ is $n a$, where $a$ is the cubic lattice constant indicated in Fig. 2. The length of the SL Brillouin zone along the growth direction is thus $\pi / n a$. This is $1 / 2 n$ times that of $2 \pi / a$, the length of the bulk BZ along [001]. This means that phonon dispersion curves in a fictitious $\operatorname{Si}(n) \operatorname{Si}(n)$ superlattice would correspond to bulk Si dispersion curves 'folded' $2 n$ times. This concept is helpful in understanding the results of the realistic calculations presented in Fig. 8 and in Fig. 9 .

Figure 8 shows the unit cell and atomic position for $\mathrm{SL}(2,2)[001]$. There are 8 atoms in the SL unit cell, leading to 24 phonon branches, the 3 lowest being the acoustic branches. Also presented in that figure are the phonon dispersion curves (with atomic relaxation effects included) for low frequency phonons along the growth direction. The size of the Brillouin zone is a quarter of the bulk zone. Thus each bulk branch gets 

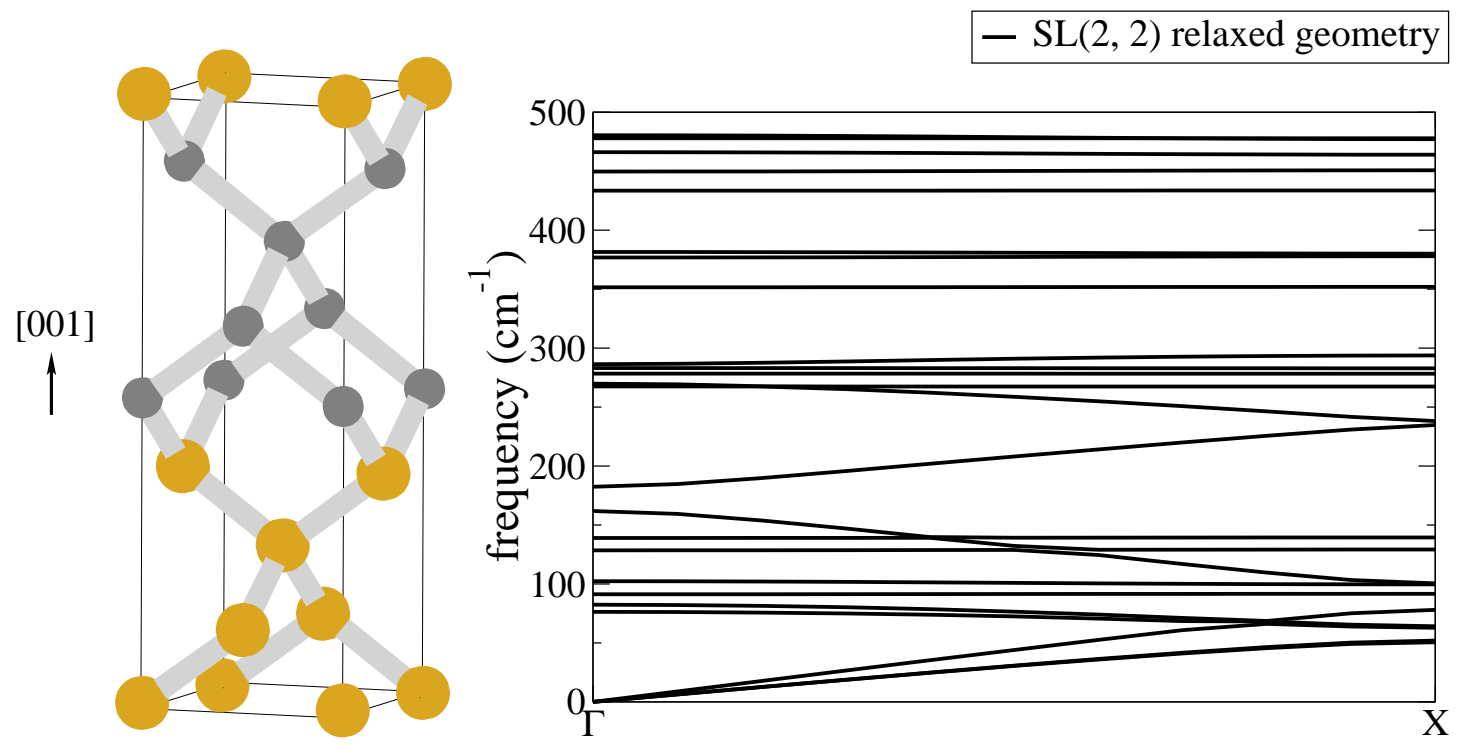

Figure 8. (left panel:) Schematic illustration of the unit cell for the $\mathrm{Si}(2) \mathrm{Ge}(2)[001]$ superlattice, comprised of $n=m=2$ bilayers of Si and Ge each oriented along the growth direction [001]. Positions of Si and Ge atoms are indicated by spheres of different sizes (and colours). (Right panel:) Dispersion curves along the growth direction for low frequency phonon branches.

folded into four branches. The foldings are accompanied by mini gap openings. The acoustic modes arise from a combination of atomic vibrations in both the $\mathrm{Si}$ and $\mathrm{Ge}$ layers. For higher branches some of the phonon modes are more Si-like and some are more Ge-like. It is instructive to examine these features by comparing the SL results with the bulk results in Fig. 3. Note the unit conversion relations: $1 \mathrm{THz}=4.1357$ $\mathrm{meV}=33.3563 \mathrm{~cm}^{-1}$.

Figure 9 shows the results for $\mathrm{SL}(1,1)$ and $\mathrm{SL}(4,4)$. The three general features along the growth direction [001], viz. zone folding, formation of mini gaps and mode confinement, can be noted for both systems. The enlargement of periodicity along the growth direction also causes the above characteristics to be present to some extent in the planar directions, such as [110]. The most significant changes in the dispersion curves and DOS can be noted for frequencies above $200 \mathrm{~cm}^{-1}$. The presence of two materials wirhin each unit cell leads to atomic relaxation, i.e. Si-Si and Ge-Ge bond length well inside Si and Ge layers and the Si-Ge interface bond length being close to the weighted average of individual bond lengths. The effect of relaxaed atomic geometry is to shift the maximum frequency upwards, and while the general qualitative structure of the DOS remains unaffected, most of the peaks above $200 \mathrm{~cm}^{-1}$ are shifted upwards. Computed phonon speeds for the acoustic branches are: $c$ (acoustic) $=6078,3614,(4633$, 4753, 4821, 5035, 5000) $\mathrm{ms}^{-1}$ for Si bulk, Ge bulk and SL structures with $n=m=1-5$.

Figure 10 presents the dispersion curves along the growth direction of the $\operatorname{Si}(4$ $\mathrm{nm}) \mathrm{Si}_{0.4} \mathrm{Ge}_{0.6}(8 \mathrm{~nm})[001]$ superlattice. Ezzahri et al. [91] successfully fabricated such a structure and, using a sub-picosecond spectroscopic technique, measured three LA mini 
SL(1,1)

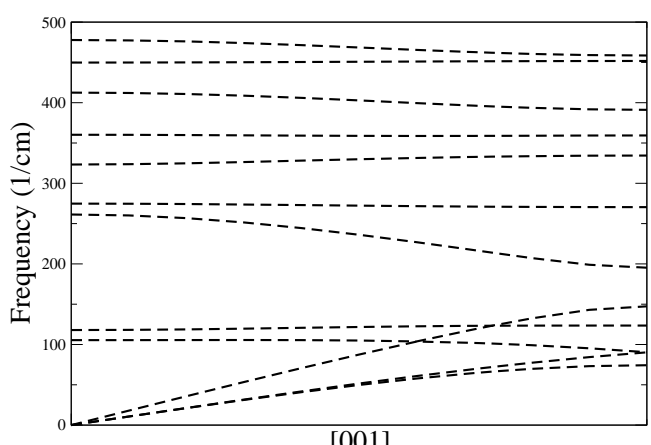

$\operatorname{SL}(4,4)$

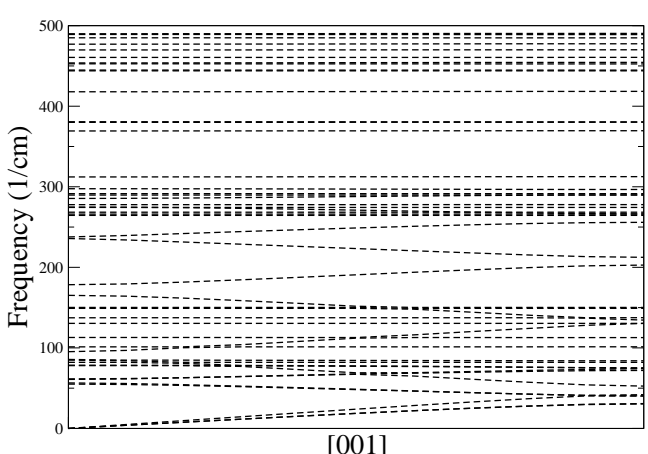

$\operatorname{SL}(1,1)$

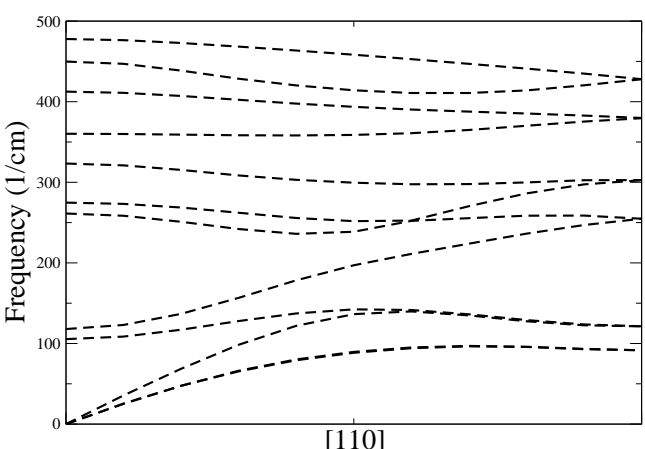

SL $(4,4)$

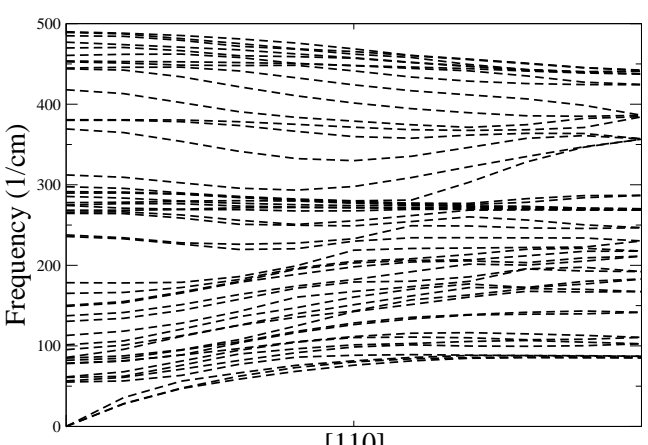

[110]

Figure 9. Phonon dispersion curves for $\mathrm{Si}(1) \mathrm{Ge}(1)[001]$ and $\mathrm{Si}(4) \mathrm{Ge}(4)[001]$ superlattices. The index $n$ in $\operatorname{Si}(n) \operatorname{Ge}(n)$ refers to the number of atomic bilayers.

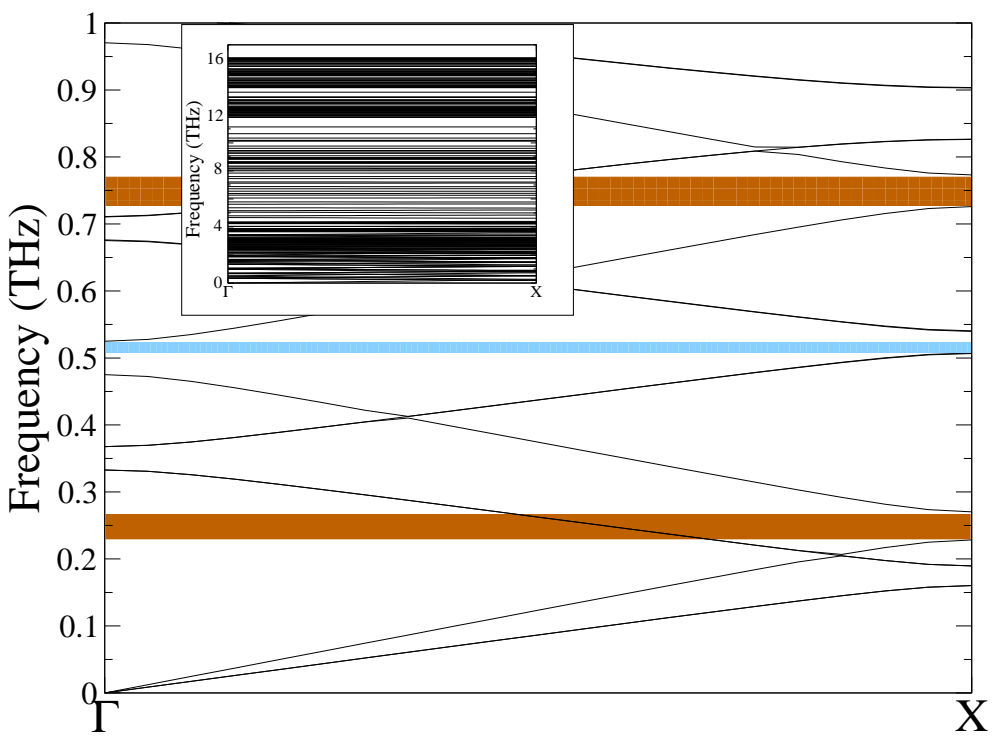

Figure 10. Phonon dispersion curves for a $\mathrm{Si}(4 \mathrm{~nm}) \mathrm{Si}_{0.4} \mathrm{Ge}_{0.6}(8 \mathrm{~nm})[001]$ superlattice. The inset shows the full range for the frequency spectra. The mini gap regions are highlighted, with the central region showing a true phononic gap. Taken with permission from Ref. [92]. 
band gaps: a gap of $36 \mathrm{GHz}$ at $145 \mathrm{GHz}$, a gap of $46 \mathrm{GHz}$ at $283 \mathrm{GHz}$ and a gap of $30 \mathrm{GHz}$ at $527 \mathrm{GHz}$. The BCM calculations by Hepplestone and Srivastava [92] successfully reproduce these results: zone-edge gap of $48 \mathrm{GHz}$ at $252 \mathrm{GHz}$, zone-centre gap of $40 \mathrm{GHz}$ at $495 \mathrm{GHz}$ and zone-edge gap of $30 \mathrm{GHz}$ at $805 \mathrm{GHz}$. In addition, the calculations also reveal three TA gaps: zone-edge gap of $30 \mathrm{GHz}$ at $175 \mathrm{GHz}$, zone-centre gap of $35 \mathrm{GHz}$ at $350 \mathrm{GHz}$ and zone-edge gap of $33 \mathrm{GHz}$ at $523 \mathrm{GHz}$. The calculations also predict that this system is a true one-dimensional phononic crystal: there exists a small frequency range of $8 \mathrm{GHz}$, between $507 \mathrm{GHz}$ and $515 \mathrm{GHz}$, that is an absolute phononic gap, in which phonons of neither polarisation can propagate along [001]. The computed speeds of the TA and LA branches are $4.18 \mathrm{~km} / \mathrm{s}$ and $6.00 \mathrm{~km} / \mathrm{s}$, respectively. In general, due to confinement within different atomic layers, optical branches (with frequencies greater than $4 \mathrm{THz}$ ) show very flat dispersion curves (i.e. are characterised with very low group velocities).

The results presented above provide clear evidence that phonon dispersion relations can be tuned via controlled fabrications of reduced-dimensional and nanocomposite structures.

\subsection{Theories of lattice thermal conductivity}

The lattice thermal (i.e. phonon) conductivity tensor $\left\{\kappa_{i j}\right\}$, defined from Eq. (10) for small temperature gradient $\boldsymbol{\nabla} T$ and under zero electric current condition $(\boldsymbol{J}=0)$, is generally expressed using Fourier's law

$$
Q_{i}=-\sum_{j} \kappa_{i j} \nabla T_{j}
$$

Theoretical approaches at two different levels of sophistication have been followed to express $\boldsymbol{\kappa}$. At one level, a statistical mechanical approach, known as the Green-Kubo linear-response approach [93], is used to express $\boldsymbol{\kappa}$ in terms of the time integral of the heat current autocorrelation function (i.e. canonical-ensemble average with respect to the Hamiltonian of the system) $<\boldsymbol{Q}(t) \cdot \boldsymbol{Q}(0)>$. This approach has been followed both at the classical and quantum levels. At another level, $\boldsymbol{\kappa}$ is expressed by obtaining a solution of a linearised Boltzmann equation satisfied by the phonon distribution function $n_{\boldsymbol{q}}(\boldsymbol{r}, t)$ in the steady state of heat flow through the solid (see, e.g. [35, 48]). In general, even the linearised phonon Boltzmann equation cannot be solved exactly, forcing development of several approximation for expressing $\boldsymbol{\kappa}$ (see Ref. [48] for a detailed discussion). Here we will provide a brief overview of the theories for phonon conductivity based on the GreenKubo's linear-response approach and the linearised Boltzmann equation formulation.

\subsubsection{Theories based on the Green-Kubo linear-response approach The Green-Kubo} approach has been attempted at both the classical and quantum levels.

(i) Classical level: 
At the classical level of the Green-Kubo approach a molecular dynamical simulation is performed in real space (see, e.g. [94, 95]). In this approach, Newton's second law and the kinematic equations of motion are used, based on (semi)classically derived interatomic potential(s), to determine the classical position and momentum space trajectories of a system of particles.

The heat current in an isotropic system is written as

$$
\boldsymbol{Q}=\sum_{i} E_{i} \boldsymbol{v}_{i}+\frac{1}{2} \sum_{i, j}\left(\boldsymbol{F}_{i j} \cdot \boldsymbol{v}_{i}\right) \boldsymbol{r}_{i j},
$$

where $E_{i}, \boldsymbol{r}_{i}$, and $\boldsymbol{v}_{i}$ are, respectively, the energy, position vector, and velocity of particle $i, \boldsymbol{r}_{i j}$ is the interparticle separation vector, and $\boldsymbol{F}_{i j}$ is the force between particles $i$ and $j$. For a monatomic bulk crystal, the heat current autocorrelation function is fitted into a functional form

$$
\langle\boldsymbol{Q}(t) \cdot \boldsymbol{Q}(0)\rangle=A \exp (-t / \tau),
$$

where $A$ is a constant and $\tau$ is a time constant. The isotropic thermal conductivity expression for a bulk material is then obtained as

$$
\kappa=\frac{k_{\mathrm{B}} T^{2} N_{0} \Omega}{3} A \tau
$$

where $k_{\mathrm{B}}$ is Boltzmann's constant, $N_{0} \Omega$ represents crystal volume (with $N_{0}$ unit cells, each of volume $\Omega$ ). The energy $E$ and force $\boldsymbol{F}$ terms are usually obtained from the use of empirically derived inter-atomic potentials. Details on the application of the approach can be found in McGaughey and Kaviany [94], and Huang and Kaviany [96].

(ii) Quantum level:

At quantum level heat current is expressed as an operator in the Heisenberg representation

$$
\hat{\boldsymbol{Q}}(t)=\frac{1}{N_{0} \Omega} \sum_{\boldsymbol{q}_{s}} \hbar \omega(\boldsymbol{q} s) \hat{n} \boldsymbol{q}_{s}(t) \boldsymbol{c}_{s}(\boldsymbol{q}),
$$

where $\boldsymbol{c}_{s}(\boldsymbol{q})$ is the velocity of phonon mode $\boldsymbol{q} s$ with frequency $\omega(\boldsymbol{q} s)$ and $\hat{n}_{\boldsymbol{q}}$ is the phonon number operator

$$
\hat{n}_{\boldsymbol{q}_{s}}=\hat{a}_{\boldsymbol{q}_{s}}^{\dagger} \hat{a}_{\boldsymbol{q}_{s}}
$$

where $\hat{a}_{\boldsymbol{q}_{s}}^{\dagger}$ and $\hat{a}_{\boldsymbol{q}_{s}}$ are phonon creation and annihilation operators, respectively. The conductivity tensor expression, therefore, reads

$$
\kappa_{i j}=\frac{\hbar^{2}}{N_{0} \Omega k_{\mathrm{B}} T^{2}} \Re \int_{0}^{\infty} \mathrm{d} t \sum_{\boldsymbol{q}_{s} \boldsymbol{q}^{\prime} s^{\prime}} \omega(\boldsymbol{q} s) \omega\left(\boldsymbol{q}^{\prime} s^{\prime}\right) c_{s}^{i}(\boldsymbol{q}) c_{s^{\prime}}^{j}\left(\boldsymbol{q}^{\prime}\right) \mathcal{C}_{\boldsymbol{q}_{s} \boldsymbol{q}^{\prime} s^{\prime}}(t),
$$

where $c_{s}^{i}(\boldsymbol{q})$ is the $i^{\text {th }}$ component of the velocity of a phonon mode $\boldsymbol{q} s$ and

$$
\mathcal{C}_{\boldsymbol{q}_{s} \boldsymbol{q}^{\prime} s^{\prime}}(t)=\left\langle\hat{a}_{\boldsymbol{q}_{s}}^{\dagger}(t) \hat{a}_{\boldsymbol{q}^{\prime} s^{\prime}}(0)\right\rangle
$$

is a correlation function. 
The correlation function $\mathcal{C}_{\boldsymbol{q}_{s} \boldsymbol{q}^{\prime} s^{\prime}}(t)$ represents the canonical-ensemble average of the operator $\hat{a}_{\boldsymbol{q}_{s}}^{\dagger}(t) \hat{a} \boldsymbol{q}^{\prime} s^{\prime}(0)$ with respect to the total phonon Hamiltonian $H$ of the system:

$$
\mathcal{C}_{\boldsymbol{q}_{s} \boldsymbol{q}^{\prime} s^{\prime}}(t)=\frac{\operatorname{Tr}\left(\mathrm{e}^{-\beta H} \hat{a}_{\boldsymbol{q}_{s}}^{\dagger}(t) \hat{a} \boldsymbol{q}^{\prime} s^{\prime}(0)\right)}{\operatorname{Tr}\left(\mathrm{e}^{-\beta H}\right)}, \quad \beta=1 / k_{\mathrm{B}} T .
$$

Several techniques have been employed to evaluate the correlation function, including the Zwanzig-Mori projection operator method, the double-time Green's function method, and the imaginary-time Green's function method. Details of the first two methods can be found in Srivastava [48] and of the third method in Ziman [97]. The final solution can be expressed as

$$
\mathcal{C}_{\boldsymbol{q}_{s} \boldsymbol{q}^{\prime} s^{\prime}}(t)=\delta \boldsymbol{q}^{\prime} \delta s s^{\prime} \bar{q}_{s}\left(\bar{n}_{\boldsymbol{q}_{s}}+1\right) \mathrm{e}^{-t / \tau} \boldsymbol{q}_{s},
$$

where $\bar{n}_{\boldsymbol{q}}$ is the Bose-Einstein distribution function and $\tau_{\boldsymbol{q}}$ is the relaxation time for a phonon in mode $\boldsymbol{q} s$. The Hamiltonian required for the simulation is usually adopted from an empirically chosen inter-atomic potential, but it can be made from first-principles treatments.

(iii) Extraction of relaxation time:

An effective relaxation time $\tau \boldsymbol{q}_{s}$ can be obtained for different situations of the change in the Hamiltonian from that for a perfect crystal within the harmonic approximation, e.g. due to the presence of impurities and defects, and crystal anharmonicity. However, there are genuine difficulties in dealing with these two situations in particular. First, the contribution towards $\tau_{\boldsymbol{q}}$ from the presence of isotopic impurities requires the molecular dynamics simulation to be carried out over an excessively large unit cell containing atoms of appropriate atomic masses. Such contributions, therefore, have not yet been included satisfactorily. Second, it is not easy to unscramble the anharmonic contribution in the form of separate phonon contributions involved in three-phonon or four-phonon processes. Neither is it easy to establish separate roles of the anharmonic Normal (momentum conserving) and Umklapp (momentum non-conserving) processes.

4.3.2. Theories based on phonon Boltzmann transport equation The Boltzmann transport equation works for classical particles and wave packets, and thus requires phonons to be treated in a simlar fashion. In essence this means that the occupation number of a phonon in mode $\boldsymbol{q} s$ is considered to be goverened by a distribution function $n_{\boldsymbol{q}_{s}}(\boldsymbol{r}, t)$ in the neighbourhood of space position $\boldsymbol{r}$ at time $t$. Such an assumption can be safely made for a phonon as long as its mean free path $\Lambda$ is not shorter than its wavelength $\lambda$. With this proviso the general form of phonon Boltzmann equation, for a solid subjected to a (small) temperature gradient $\nabla T$, can be written as

$$
-\boldsymbol{c}_{s}(\boldsymbol{q}) \cdot \boldsymbol{\nabla} T \frac{\partial n_{\boldsymbol{q}}}{\partial T}+\left.\frac{\partial n_{\boldsymbol{q}}}{\partial t}\right|_{\text {scatt }}=0
$$


with the second term on the left-hand side representing the rate of change due to phonon scattering mechanisms. A physically appealing simplification of Eq. (75) is the linearised Boltzmann equation

where

$$
-\boldsymbol{c}_{s}(\boldsymbol{q}) \cdot \boldsymbol{\nabla} T \frac{\partial \bar{n} \boldsymbol{q}_{s}}{\partial T}+\left.\frac{\partial n \boldsymbol{q}_{s}}{\partial t}\right|_{\text {scatt }}=0
$$

$$
\bar{n}_{\boldsymbol{q}_{s}}=\left[\exp \left(\hbar \omega(\boldsymbol{q} s) / k_{\mathrm{B}} T\right)-1\right]^{-1}
$$

is the Bose-Einstein (or equilibrium) distribution function for phonons. An exact analytic solution of the Boltzmann equation (76) can only be achieved under certain simplifying conditions [35]. In general, an appropriate expression for the term $\partial n_{\boldsymbol{q}_{s}} /\left.\partial t\right|_{\text {scatt }}$, contributed by all relevant phonon scattering mechanisms, must be derived before Eq. (76) can solved for $n_{\boldsymbol{q}_{s}}(t)$ and an expression for thermal conductivity established. In general, phonon scattering mechanisms can be described as elastic (in which the participating phonon $\boldsymbol{q} s$ retains its identity) and inelastic (in which the participating phonon $\boldsymbol{q} s$ loses its identity). In general, only approximate forms of inelastic scattering rates can be derived. This is particularly the case for phonon scattering due to crystal anharmonic effects. This difficulty has led to two main routes for the derivation of an expression for the thermal conductivity. A detailed discussion is provided in Ref. [48], but we will briefly outline them here.

(i) Variational principles:

In equilibrium the phonon distribution function does not change with time, i.e. $\partial \bar{n} \boldsymbol{q}_{s} / \partial t=0$. The solution to this equation is provided by the Bose-Einstein function in Eq. (77). In the presence of a finite temperature gradient the Bose-Einstein distribution function can no longer describe the phonon distribution function. Let us introduce a function $\psi_{\boldsymbol{q}}$, defined in terms of a displaced Bose-Einstein distribution function

$$
\begin{aligned}
& n_{\boldsymbol{q}_{s}}=\left[\exp \left(\hbar \omega(\boldsymbol{q} s) / k_{\mathrm{B}} T-\psi_{\boldsymbol{q}_{s}}\right)-1\right]^{-1} \\
& \simeq \bar{n} \boldsymbol{q}_{s}-\left.\psi \boldsymbol{q}_{s} \frac{\partial n_{\boldsymbol{q}_{s}}}{\partial\left(\hbar \omega(\boldsymbol{q} s) / k_{\mathrm{B}} T-\psi_{\left.\boldsymbol{q}_{s}\right)}\right.}\right|_{\psi} \boldsymbol{q}_{s}=0 \\
& =\bar{n} \boldsymbol{q}_{s}-\psi_{\boldsymbol{q}_{s}} \frac{\partial \bar{n}_{\boldsymbol{q}_{s}}}{\partial\left(\hbar \omega(\boldsymbol{q} s) / k_{\mathrm{B}} T\right)} \\
& =\bar{n} \boldsymbol{q}_{s}+\psi_{\boldsymbol{q}_{s}} \bar{n}_{\boldsymbol{q}}\left(\bar{n} \boldsymbol{q}_{s}+1\right) .
\end{aligned}
$$

The function $\psi_{\boldsymbol{q}}$ is a measure of the deviation from equilibrium in the phonon distribution, and the function $\psi_{\boldsymbol{q}_{s}} k_{\mathrm{B}} T$ is the average extra energy acquired by the phonon mode $\boldsymbol{q} s$ during the transport process. Equation (79) was used to define $\psi_{\boldsymbol{q}_{s}}$ in Refs. [35, 98]. An specific form of the displaced Bose-Einstein function distribution was considered in Refs. [99, 100], which we will discuss in the next sub-section. Using the above notation, we can express the scattering term in Eq. (76) as

$$
-\left.\frac{\partial n \boldsymbol{q}_{s}}{\partial t}\right|_{\text {scatt }}=\sum_{\boldsymbol{q}^{\prime} s^{\prime}} P_{\boldsymbol{q}^{\prime}}^{s s^{\prime}} \psi_{\boldsymbol{q}^{\prime}}^{s^{\prime}}
$$


where $P_{\boldsymbol{q q}^{\prime}}^{s \boldsymbol{q}^{\prime}}$ are the elements of the phonon collision operator, providing a measure of phonon transition probabilities (see, e.g. [35]).

For each appropriate scattering mechanism, an expression for the phonon collision operator elements $P_{\boldsymbol{q} \boldsymbol{q}^{\prime}}^{s^{\prime}}$ can be derived by applying time dependent perturbation theory. The deviation function $\psi_{\boldsymbol{q}_{s}}$ can then be obtained from Eq. (76) provided that the inverse of the matrix operator $\left\{P_{\boldsymbol{q} \boldsymbol{q}^{\prime}}^{s s^{\prime}}\right\}$ exists. Unfortunately, only partial information is available about the nature of the anharmonic part of the collision operator (for details, see $[48,101])$. This leaves $\psi \boldsymbol{q}_{s}$ unknown in the temperature range where the role of crystal anharmonicity plays an important role. The essence of the variational method for lattice thermal conductivity is to treat $\psi_{\boldsymbol{q}_{s}}$ as a trial function. The simplest approximation for the anharmonic contribution to $\psi_{\boldsymbol{q}_{s}}$ is $[35,48,101,98]$

$$
\psi_{\boldsymbol{q}}=\boldsymbol{q} \cdot \boldsymbol{u}
$$

where $\boldsymbol{u}$ is some constant vector parallel to the applied temperature gradient.

Ziman [35] derived a lower bound for the conductivity $\kappa$ by using the simple trial function in Eq. (81). By noting and employing the positive semi-definite property of the phonon collision operator $P$, Benin [102] developed a sequence of monotonically convergent lower bounds for the conductivity $\kappa$. The first term in this sequence is the Ziman limit. It was later shown by Srivastava [103] that a sequence of monotonically convergent upper bounds for the conductivity can also be developed. In theory, an estimate for the exact conductivity can then be confined to a small difference between an upper bound and a lower bound. An improved estimate of any conductivity bound can be made by using a more general form of the trial function $\psi_{\boldsymbol{q}_{s}}$ than in Eq. (81) and adopting the scaling and Ritz procedures [98, 104]. The concept of obtaining both a lower bound and an upper bound for estimating a desired (but inherently unkown) quantity is called the complementary variational principles, detailes of which can be found in the book by Arthurs [105] and in the review article by Srivastava and Hamilton [106].

\section{(ii) Relaxation-time theories:}

As mentioned earlier, a possible solution for the deviation function $\psi_{\boldsymbol{q}_{s}}$ requires the difficult task of obtaining an acceptable form of the phonon collision operator $P$ that can be inverted. This difficulty is dealt with by expressing the scattering rate in Eq. (76) with the introduction of the concept of a phonon relaxation time. This is achieved by expressing

$$
-\left.\frac{\partial n_{\boldsymbol{q}_{s}}}{\partial t}\right|_{\text {scatt }}=\frac{n_{\boldsymbol{q}_{s}}-\bar{n}_{\boldsymbol{q}_{s}}}{\tau_{\boldsymbol{q}_{s}}}
$$

where $\tau_{\boldsymbol{q}}$ is the relaxation time for a phonon in mode $\boldsymbol{q} s$. The phonon conductivity tensor can be expressed as

$$
\kappa_{i j}=\frac{\hbar^{2}}{N_{0} \Omega k_{B} T^{2}} \sum_{\boldsymbol{q}_{s}} \omega^{2}(\boldsymbol{q} s) c_{s}^{i}(\boldsymbol{q}) c_{s}^{j}(\boldsymbol{q}) \tau(\boldsymbol{q} s) \bar{n}(\boldsymbol{q} s)(\bar{n}(\boldsymbol{q} s)+1) .
$$


For an isotropic system the conductivity expression can be written, using Eqs. (76) and (82), as

$$
\begin{aligned}
\kappa & =\frac{\hbar^{2}}{3 N_{0} \Omega k_{B} T^{2}} \sum_{\boldsymbol{q}_{s}} \omega^{2}(\boldsymbol{q} s) c_{s}^{2}(\boldsymbol{q}) \tau(\boldsymbol{q} s) \bar{n}(\boldsymbol{q} s)(\bar{n}(\boldsymbol{q} s)+1), \\
& =\frac{\hbar}{N_{0} \Omega|\nabla T|^{2}} \sum_{\boldsymbol{q}_{s}} \omega(\boldsymbol{q} s)\left(\boldsymbol{c}_{s} \cdot \boldsymbol{\nabla} T\right) \psi_{\boldsymbol{q}_{s}} \bar{n}(\boldsymbol{q} s)(\bar{n}(\boldsymbol{q} s)+1) .
\end{aligned}
$$

It is important to note that the validity of the relaxation-time approach is limited by the Landau-Peierls-Ziman condition [35]

$$
\omega \tau>1 ; \quad \text { or } \Lambda>\lambda,
$$

where $\Lambda$ and $\lambda$ are the phonon mean-free path and wavelength, respectively. Thus the Boltzmann-equation based relaxation time approach is unsuitable for applications to samples thinner than average phonon wavelength.

\section{(a) Single-mode relaxation-time theory:}

In its simple form the single-mode relaxation time (smrt) approximation assumes that in a three-phonon event $\boldsymbol{q} s \pm \boldsymbol{q}^{\prime} s^{\prime} \rightleftharpoons \boldsymbol{q}^{\prime \prime} s^{\prime \prime}$ only the phonon mode $\boldsymbol{q} s$ is described by a displaced distribution and the other two modes obey the equilibrium distribution. Such a description can be justified to some extent [107]. Essentially, $\tau_{\text {smrt }}$ can be derived from the diagonal part of the phonon collision operator $P$ :

$$
\tau_{\boldsymbol{q}_{s, \mathrm{smrt}}}^{-1}=\frac{P_{\boldsymbol{q} \boldsymbol{q}}^{s s}}{\bar{n}(\boldsymbol{q} s)(\bar{n}(\boldsymbol{q} s)+1)} .
$$

Within this approximation, the isotropic conductivity expression then reads

$$
\kappa_{\mathrm{smrt}} \equiv \kappa_{\mathrm{D}}=\frac{\hbar^{2}}{3 N_{0} \Omega k_{B} T^{2}} \sum_{\boldsymbol{q}_{s}} \omega^{2}(\boldsymbol{q} s) c_{s}^{2}(\boldsymbol{q}) \tau(\boldsymbol{q} s)_{\mathrm{smrt}} \bar{n}(\boldsymbol{q} s)(\bar{n}(\boldsymbol{q} s)+1) .
$$

(b) Callaway's effective relaxation-time theory:

Several attempts have been made to modify the single-mode relaxation time scheme, including the works of Klemens [100], Callaway [108], Hamilton [109], Simons [110], and Srivastava [111]. Of these, the best known and adopted scheme is that of Callaway [108]. Here we follow Parrott [112] to derive a generalisation of the Callaway thermal conductivity equation.

It was noted by Klemens [124] that momentum conserving (Normal, or N) phononphonon interaction processes tend to restore a given non-equilibrium distribution $n(\boldsymbol{q} s)$ not to the equilibrium distribution $\bar{n}(\boldsymbol{q} s)$ in Eq. (79) with $\psi_{\boldsymbol{q}_{s}}=0$ but towards a distribution of the form

$$
n_{\boldsymbol{q}_{s}}(\boldsymbol{u})=\left[\exp \left(\hbar \omega(\boldsymbol{q} s) / k_{\mathrm{B}} T-\boldsymbol{q} \cdot \boldsymbol{u}\right)-1\right]^{-1},
$$

where $\boldsymbol{u}$ is a constant vector parallel to the temperature gradient (as also considered in Eq. (81)). With this information, the basic assumption of the Callaway theory is to express

$$
\left.\frac{\partial n_{\boldsymbol{q}} s}{\partial t}\right|_{N}=\frac{\boldsymbol{q} \cdot u-\psi \boldsymbol{q}_{s}}{\tau_{N, \boldsymbol{q}}} \bar{n}(\boldsymbol{q} s)(\bar{n}(\boldsymbol{q} s)+1)
$$


Since momentum is conserved in $\mathrm{N}$ processes, i.e.

$$
\begin{gathered}
\left.\sum_{\boldsymbol{q}_{s}} \boldsymbol{q} \frac{\partial{ }^{\prime} \boldsymbol{q}_{s}}{\partial t}\right|_{N}=0, \\
\left.\sum_{\boldsymbol{q}_{s}}(\boldsymbol{q} \cdot \boldsymbol{u}) \frac{\partial n \boldsymbol{q}_{s}}{\partial t}\right|_{N}=0 .
\end{gathered}
$$

Eq. (92) follows from Eq. (91), since if $\boldsymbol{A}=0$ then $\boldsymbol{A} \cdot \boldsymbol{B}=0$ for any $\boldsymbol{B}$. From Eq. (92) we can express

$$
u \equiv|\boldsymbol{u}|=\frac{\sum \boldsymbol{q}_{s} \psi \boldsymbol{q}_{s} q \cos \theta \tau_{N, \boldsymbol{q}_{s}}^{-1} \bar{n}(\boldsymbol{q} s)(\bar{n}(\boldsymbol{q} s)+1)}{\sum \boldsymbol{q}_{s} q^{2} \cos ^{2} \theta \tau_{N, \boldsymbol{q}_{s}}^{-1} \bar{n}(\boldsymbol{q} s)(\bar{n}(\boldsymbol{q} s)+1)},
$$

where $\theta$ is the angle between $\boldsymbol{q}$ and $\boldsymbol{\nabla}$ T. With Eq. (90), the phonon Boltzmann transport equation can be expressed as

$$
\begin{aligned}
& -\frac{\hbar \omega(\boldsymbol{q} s)}{k_{\mathrm{B}} T^{2}} c_{s} \hat{\boldsymbol{q}} \cdot \boldsymbol{\nabla} T \bar{n}(\boldsymbol{q} s)(\bar{n}(\boldsymbol{q} s)+1)=-\left.\frac{\partial n_{\boldsymbol{q}_{s}}}{\partial t}\right|_{\text {coll }} \\
& =-\left(\left.\frac{\partial n \boldsymbol{q}_{s}}{\partial t}\right|_{N}+\left.\frac{\partial n \boldsymbol{q}_{s}}{\partial t}\right|_{R}\right) \\
& =\left(\frac{\psi \boldsymbol{q}_{s}-\boldsymbol{q} \cdot \boldsymbol{u}}{\tau \boldsymbol{q}_{s, N}}+\frac{\psi_{\boldsymbol{q}_{s}}}{\tau_{\boldsymbol{q}_{s, R}}}\right) \bar{n}(\boldsymbol{q} s)(\bar{n}(\boldsymbol{q} s)+1),
\end{aligned}
$$

where $\mathrm{R}$ represents all momentum non-conserving (e.g. Umklapp, defect, boundary, etc.) scattering processes. Form Eq. (94) we obtain another relation between $\psi_{\boldsymbol{q}_{s}}$ and $u$ :

$$
\psi_{\boldsymbol{q}_{s}}=\tau_{\boldsymbol{q}_{s}} \cos \theta\left(q u \tau_{\boldsymbol{q}_{s, N}^{-1}}^{-1}-\frac{\hbar \omega(\boldsymbol{q} s)}{k_{\mathrm{B}} T^{2}} c_{s}|\nabla T|\right) .
$$

Inserting $\psi_{\boldsymbol{q}_{s}}$ from Eq. (95) in Eq. (93) we finally determine $u$ :

$$
u=-\frac{\hbar|\nabla T|}{k_{\mathrm{B}} T^{2}} \mathcal{A}
$$

where the temperature-dependent parameter $\mathcal{A}$ is independent of the phonon wave vector $\boldsymbol{q}$ and polarisation $s$, defined as

$$
\mathcal{A}=\frac{\sum \boldsymbol{q}_{s} q c_{s} \omega(\boldsymbol{q} s) \tau_{\boldsymbol{q}_{s}} \tau_{\boldsymbol{q}_{s, N}}^{-1} \bar{n}(\boldsymbol{q} s)(\bar{n}(\boldsymbol{q} s)+1)}{\sum \boldsymbol{q}_{s} q^{2} \tau_{\boldsymbol{q}_{s, N}^{-1}}^{-1}\left(1-\tau_{\boldsymbol{q}_{s}} \tau^{-1} \boldsymbol{q}_{s, N}\right) \bar{n}(\boldsymbol{q} s)(\bar{n}(\boldsymbol{q} s)+1)} .
$$

Substituting Eq. (96) Eq. (95) we obtain the form of the deviation function $\psi_{\boldsymbol{q}_{s}}$ in the Callaway theory

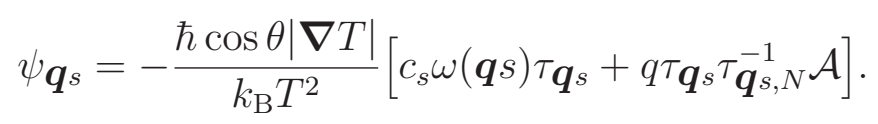

The following Callaway conductivity formula can be obtained by substituing Eq. (98) in Eq. (85)

$$
\begin{aligned}
\kappa_{C}= & \frac{\hbar^{2}}{3 N_{0} \Omega k_{\mathrm{B}} T^{2}} \times \\
& {\left[\sum_{\boldsymbol{q}_{s}} c_{s}^{2} \omega^{2}(q s) \tau \boldsymbol{q}_{s} \bar{n}(\boldsymbol{q} s)(\bar{n}(\boldsymbol{q} s)+1)+\mathcal{A} \sum_{\boldsymbol{q}_{s}} q c_{s} \omega(\boldsymbol{q} s) \tau_{q s} \tau_{\boldsymbol{q}_{s, N}}^{-1} \bar{n}(\boldsymbol{q} s)(\bar{n}(\boldsymbol{q} s)+1)\right] } \\
= & \kappa_{\mathrm{D}}+\kappa_{\mathrm{Ndrift}} .
\end{aligned}
$$


In the above formula the first term is the Debye term (viz. the smrt result presented in Eq. (85)) and the second term is the so-called N-drift term arising from the momentum conserving nature of the Normal processes. Note that the generalised Callaway formula in Eq. (99) is essentially the same as in Parrott's paper [112] but clarifies that both the Debye term $\kappa_{\mathrm{D}}$ and the $\mathrm{N}$-drift term $\kappa_{\mathrm{Ndrift}}$ are contributed by separate phonon polarisations.

\section{(iii) An iterative scheme:}

In an attempt to include contributions beyond the diagonal part of the phonon collision operator (which defines $\tau_{\text {smrt }}$ ), Omini and Sparavigna [113] employed an iterative scheme. This scheme has been followed up by Broido et al. [114]. P qq $_{\boldsymbol{q}}^{\text {ds }}$ defined in Eq. (87) is considered for the zeroth-order solution of the Boltzmann equation. Substitution of this into Eq. (80) yields the first-order solution for the function $\psi_{\boldsymbol{q}_{s}}$, and functions $\psi_{\boldsymbol{q}^{\prime} s^{\prime}}$ and $\psi_{\boldsymbol{q}^{\prime \prime} s^{\prime \prime}}$ are obtained by interpolation of $\psi$ values taken from surrounding on-grid points. Several (typically 50) iterations are needed to obtain a converged solution of the Boltzmann equation for thermal conductivity. This iterative scheme can be viewed as an attempt to obtain an estimate of the thermal conductivity beyond the smrt result $\kappa_{\text {smrt }}$ using $\tau_{\text {smrt }}$ in Eq. (85). However, no attempt has been made by any group to examine the extent to which the converged result differs from $\kappa_{\text {smrt }}$ or $\kappa_{C}$.

\section{(iv) Numerical evaluation:}

Following the discussion in Sect. 4.1.2, the Brillouin zone summation for numerical evaluation of a thermal conductivity expression can be performed either by following the 'special $\boldsymbol{q}$-points' method or Debye's isotropic continuum method. The 'special $\boldsymbol{q}$-points' method can be straightforwardly adopted following Eq. (62). Employing Debye's isotropic continuum scheme, the conductivity expressions within the singlemode relaxation time approach $\left(\kappa_{\mathrm{smrt}}\right)$ and within the generalised Callaway formalism $\left(\kappa_{\mathrm{C}}\right)$ can be written as

$$
\begin{aligned}
\kappa_{\mathrm{smrt}} & \equiv \kappa_{\mathrm{D}}=\frac{\hbar^{2} q_{\mathrm{D}}^{5}}{6 \pi^{2} k_{B} T^{2}} \sum_{s} c_{s}^{4} \int_{0}^{1} d x x^{4} \tau \bar{n}(\bar{n}+1), \\
\kappa_{\mathrm{C}} & =\frac{\hbar^{2} q_{\mathrm{D}}^{5}}{6 \pi^{2} k_{B} T^{2}}\left[\sum_{s} c_{s}^{4} \int_{0}^{1} d x x^{4} \tau \bar{n}(\bar{n}+1)+\mathcal{A} \sum_{s} c_{s}^{2} \int_{0}^{1} d x x^{4} \tau \tau_{N}^{-1} \bar{n}(\bar{n}+1)\right],
\end{aligned}
$$

where $x=q / q_{\mathrm{D}}$ with $q_{\mathrm{D}}$ representing the Debye radius, $\bar{n}=\bar{n}(x, s)$ and $\mathcal{A}$ expressed as

$$
\mathcal{A}=\frac{\sum_{s} c_{s}^{2} \int_{0}^{1} d x x^{4} \tau_{s} \tau_{s, N}^{-1} \bar{n}(\bar{n}+1)}{\sum_{s} \int_{0}^{1} d x x^{4} \tau_{s, N}^{-1}\left(1-\tau_{s} \tau_{s, N}^{-1}\right) \bar{n}(\bar{n}+1)} .
$$

As mentioned in Eqs. (99) and (100), the first and second terms in Eq. (102) represent the single-mode relaxation time result $\kappa_{\text {smrt }}$ (the Debye term $\kappa_{\mathrm{D}}$ ) and the N-drift term $\kappa_{\mathrm{N}-\mathrm{drift}}$, respectively. Note that, resulting from the momentum conservation condition for the anharmonic phonon interactions, the $\mathrm{N}$-drift term $\kappa_{\mathrm{N} \text {-drift }}$ is an addition to the smrt contribution $\kappa_{\text {smrt }}$. Numerical evaluation of phonon relaxation times $\tau$ and $\tau_{N}$ will be discussed in the next section. 


\subsection{Phonon Interactions}

The elements of the phonon collision operator $P_{\boldsymbol{q} \boldsymbol{q}^{\prime}}^{s s^{\prime}}$, and consequently the phonon relaxation rate $\tau^{-1}$, have contributions from several scattering mechanisms, depending on composition, size and dimensionality of the system.

4.4.1. Bulk materials Generally for bulk semiconductors one needs to consider scatterings from boundaries (sample size), defects (e.g. mass isotopes, point impurities, line defects, planar defects etc.), donor/acceptor carriers and anharmonicity.

\section{(i) Boundary scattering:}

Purely diffusive phonon scattering rate from crystal boundaries can be expressed as

$$
\tau_{\mathrm{bs}}^{-1}(\boldsymbol{q} s)=c_{s} / L_{0} .
$$

Here $L_{0}$ denotes an effective boundary length, which depends on the geometrical shape of crystal. $L_{0}=D$ for a crystal of cylindrical shape with circular cross-section of diameter $D$, and $L_{0}=1.12 d$ for a square cross-section of side $d$. For polycrystalline solids $L_{0}$ is a measure of an effective grain size.

In reality, consideration of the polish quality of crystal surface must be made and an effective boundary length determined. This can be quite a difficult task, knowing especially that the precise nature of surface roughness and structure of grown samples is usually ill-defined. A phenomenological model [35] allows us to define an effectively longer boundary length $L$ by incorporating the surface polish quality in terms of the specularity factor $p$,

$$
L=\frac{(1+p)}{(1-p)} L_{0}
$$

The limiting cases $p=0$ and $p=1$ are purely diffusive (or Casimir) scattering and purely specular boundary scattering (or reflection), respectively. For a given specularity factor $p$, each phonon is reflected $1 /(1-p)$ times before being diffusively scattered.

(ii) Isotopic mass-defect scattering:

Consider a crystal with an average mass per unit cell as $\bar{M}$ and let $f_{i}$ be the fraction of the unit cells containing the isotopic mass $M_{i}$. The scattering rate of a phonon mode $\boldsymbol{q} s$ due to isotopic mass defects is $[107,48]$

$$
\tau_{\mathrm{md}}^{-1}(\boldsymbol{q} s)=\frac{\Gamma_{m d} \pi}{6 N_{0}} \omega^{2}(\boldsymbol{q} s) g(\omega(\boldsymbol{q} s))
$$

where $g(\omega)$ is the density of states and $\Gamma_{m d}$ is the isotopic mass parameter. Within the isotropic continuum approximation, the expression in Eq. (106) reads

$$
\begin{aligned}
\tau_{\mathrm{md}}^{-1}(\boldsymbol{q} s) & =\frac{\Gamma_{\mathrm{md}} \Omega}{4 \pi \bar{c}^{3}} \omega^{4}(\boldsymbol{q} s), \\
& =A_{\mathrm{md}} \omega^{4}(\boldsymbol{q} s),
\end{aligned}
$$


with $\bar{c}$ as the average acoustic phonon speed. For a single-species crystal the isotopic mass parameter is evaluated as

$$
\Gamma_{\mathrm{md}}=\sum_{i} f_{i}\left(\Delta M_{i} / \bar{M}\right)^{2},
$$

where $\bar{M}$ is the average atomic mass, $f_{i}$ is the frequency of the $i^{\text {th }}$ isotope of mass $M_{i}$, and $\Omega$ is the unit cell volume. Obviously, for a monatomic crystal, with one atom per unit cell, $\Omega$ represents the atomic volume.

(iii) Alloy scattering:

In alloys there will be additional mass-disorder scattering. Let us denote a single crystal alloy in the form of a composite material with molecular formula $A_{x} B_{y} C_{z} \ldots$ With this consideration we can express $\Gamma_{\mathrm{md}}$ as $[115]$

$$
\begin{aligned}
\Gamma_{\mathrm{md}}^{\text {alloy }} & =\frac{x}{(x+y+z+\ldots)}\left(\frac{M_{A}}{\bar{M}}\right)^{2} \Gamma(A) \\
& +\frac{y}{(x+y+z+\ldots)}\left(\frac{M_{B}}{\bar{M}}\right)^{2} \Gamma(B) \\
& +\frac{z}{(x+y+z+\ldots)}\left(\frac{M_{C}}{\bar{M}}\right)^{2} \Gamma(C) \\
& +\ldots,
\end{aligned}
$$

with

$$
\Gamma(A)=\sum_{i} f_{i}\left(\frac{\Delta M_{i}(A)}{\bar{M}_{A}}\right)^{2}
$$

etc. and

$$
\bar{M}=\frac{x M_{A}+y M_{B}+z M_{C}+\ldots}{x+y+z+\ldots} .
$$

(iv) Scattering with donor electrons:

Consider the scattering of phonons with donor electrons in doped semiconductors. We may consider a local displacement field $\boldsymbol{u}(\mathrm{r})$ produced by longitudinal acoustic phonons, causing an energy change of the form $E_{\text {def }}=C \Delta(\boldsymbol{r})=C_{1} \nabla \cdot \boldsymbol{u}(\mathrm{r})=C_{2} \hat{\boldsymbol{q}} \cdot \boldsymbol{e}_{\boldsymbol{q}}$, with $C_{1}$ and $C_{2}$ as some parameters. The matrix element of the deformation potential $E_{\text {def }}$ can be evaluated by expressing the donor electron wave function as a Bloch function. Application of Fermi's golden rule results in the following expression for the relaxation rate of a phonon mode $\boldsymbol{q} s$ (see $[116,117]$ for details)

$$
\tau^{-1}(\boldsymbol{q} s)=\frac{m^{\star 2} E_{\mathrm{def}}^{2} k_{\mathrm{B}} T}{2 \pi \varrho c_{\mathrm{L}} \hbar^{4}}\left[z-\ln \left(\frac{1+\exp \left(\xi-\zeta+z^{2} / 16 \xi+z / 2\right)}{1+\exp \left(\xi-\zeta+z^{2} / 16 \xi-z / 2\right)}\right)\right],
$$

with $c_{\mathrm{L}}$ is the speed of longitudinal acoustic phonons, $z=\hbar \omega / k_{\mathrm{B}} T$ and $\xi=m^{\star} c_{\mathrm{L}}^{2} / 2 k_{\mathrm{B}} T$. This expression can be reduced to the following form in the case of a heavily doped, degenerate, semiconductor with $\zeta>E_{0}$ and $\zeta-E_{0}>>k_{\mathrm{B}} T$, where $E_{0}=\hbar^{2} \omega^{2} / 8 m^{\star} c_{\mathrm{L}}^{2}+$ $\frac{1}{2} m^{\star} c_{\mathrm{L}}^{2}-\hbar \omega / 2$,

$$
\tau^{-1}(\boldsymbol{q} s)=\frac{m^{\star 2} E_{\mathrm{def}}^{2}}{2 \pi \varrho \hbar^{3} c_{\mathrm{L}}} \omega .
$$


For moderately doped semiconductors, Parrott [118] reduced Eq. (112) to a simpler form. For small carrier concentration $n_{\mathrm{c}}$ we can expand $\tau^{-1}(\boldsymbol{q} s)$ in Taylor series about $n_{\mathrm{c}}=0$. Remembering the relationship between $n_{\mathrm{c}}$ and the reduced Fermi energy $\zeta$ as [39] $n_{\mathrm{c}}=2\left(m^{\star} k_{\mathrm{B}} T / 2 \pi \hbar^{2}\right)^{3 / 2} \exp (\zeta)=U_{\mathrm{c}} e^{\zeta}$ one obtains from Eq. (112)

$$
\left(\frac{\mathrm{d}\left(\tau^{-1}\right)}{\mathrm{d} n_{c}}\right)_{n_{\mathrm{c}}=0}=\frac{E_{\mathrm{def}}^{2} m^{\star 2} k_{\mathrm{B}} T}{\pi \hbar^{4} \varrho c_{\mathrm{L}}} \times \frac{1}{2}\left(\frac{2 \pi \hbar^{2}}{m^{\star} k_{\mathrm{B}} T}\right)^{3 / 2} \exp \left[-\left(\xi+z^{2} / 16 \xi\right)\right] \sinh (z / 2) .(11
$$

Substituting for $U_{\mathrm{c}}$ one thus finds

$$
\tau^{-1}(\boldsymbol{q} s)=\frac{2 n_{\mathrm{c}} E_{\mathrm{def}}^{2} \sqrt{\pi \xi}}{\hbar \varrho c_{\mathrm{L}}^{2}} \exp \left[-\left(\xi+z^{2} / 16 \xi\right)\right] \sinh (z / 2),
$$

which for $z \rightarrow 0$ becomes

$$
\tau^{-1}(\boldsymbol{q} s)=\frac{n_{\mathrm{c}} E_{\mathrm{def}}^{2} \omega}{\varrho c_{\mathrm{L}}^{2} k_{\mathrm{B}} T} \sqrt{\frac{\pi m^{*} c_{\mathrm{L}}^{2}}{2 k_{\mathrm{B}} T}} \exp \left(\frac{-m^{*} c_{\mathrm{L}}^{2}}{2 k_{\mathrm{B}} T}\right) .
$$

Note that the root sign in the printed equation (2a) in Ref. [118] should cover the " $\xi$ " sign.

\section{(v) Anharmonic scattering:}

Atomic vibrations in solids become anharmonic with increase in temperature. This results in phonon-phonon interactions, which can be treated using time-dependent perturbation theory. First-principles efforts are being made to investigate three-phonon scattering rates by "extracting" third-order interatomic force constants using DFPT $[119,120]$. However, it is fair to remark that obtaining a workable expression for a crystal anharmonic potential from first principles, particularly including its temperature dependence, is an enormous task, and has not yet been achieved satisfactorily.

(a) Cubic anharmonic potential - acoustic phonons:

A workable form of cubic anharmonic crystal potential can be expressed, by treating a crystal as an isotropic anharmonic elastic continuum, as [35, 48]

$$
\begin{aligned}
\mathcal{V}_{3}^{\mathrm{ac}} & =\frac{1}{3 !} \sqrt{\frac{\hbar^{3}}{2 \varrho N_{0} \Omega}} \frac{\gamma(T)}{\bar{c}} \sum_{\boldsymbol{q}_{s} \boldsymbol{q}^{\prime} s^{\prime} \boldsymbol{q}^{\prime \prime} s^{\prime \prime}} \frac{\mathcal{B}_{\boldsymbol{q} s, \boldsymbol{q}^{\prime} s^{\prime}, \boldsymbol{q}^{\prime \prime} s^{\prime \prime}}^{\mathrm{ac}}}{\sqrt{\omega(\boldsymbol{q} s) \omega\left(\boldsymbol{q}^{\prime} s^{\prime}\right) \omega\left(\boldsymbol{q}^{\prime \prime} s^{\prime \prime}\right)}} \\
& \times\left(a_{\boldsymbol{q}_{s}}^{\dagger}-a_{-} \boldsymbol{q}_{s}\right)\left(a_{\boldsymbol{q}^{\prime} s^{\prime}}^{\dagger}-a_{-} \boldsymbol{q}^{\prime} s^{\prime}\right)\left(a_{\boldsymbol{q}^{\prime \prime} s^{\prime \prime}}^{\dagger}-a_{-} \boldsymbol{q}^{\prime \prime} s^{\prime \prime}\right) \delta_{\boldsymbol{q}_{+} \boldsymbol{q}^{\prime}+\boldsymbol{q}^{\prime \prime}, \boldsymbol{G}}
\end{aligned}
$$

with

$$
\mathcal{B}_{\boldsymbol{q}_{s,}^{\mathrm{ac}}, \boldsymbol{q}^{\prime} s^{\prime}, \boldsymbol{q}^{\prime \prime} s^{\prime \prime}}=\omega(\boldsymbol{q} s) \omega\left(\boldsymbol{q}^{\prime} s^{\prime}\right) \omega\left(\boldsymbol{q}^{\prime \prime} s^{\prime \prime}\right),
$$

where $\gamma(T)$ is a mode-average but temperature-dependent Grüneisen's constant, $\boldsymbol{G}$ is a reciprocal lattice vector, $a_{\boldsymbol{q}_{s}}^{\dagger}\left(a_{\boldsymbol{q}_{s}}\right)$ are the phonon creation(annihilation) operators, and other symbols have previously been defined. It should be remarked that the above form of the anharmonic potential assumes that the phonon spectrum consists of only acoustic branches.

(b) Cubic anharmonic potential - acoustic and optical phonons: 
Simple attempts at including the role of optical phonons in anharmonic interactions have been made by Klemens [121] and Srivastava [122]. Recently, an ad hoc form of isotropic anharmonic potential has been proposed [90, 123] that considers acoustic as well as optical phonon branches. The basic physical considerations behind this approach are rooted in the theory advocated by Klemens [124], and Ridley and Gupta [125]. In this approach the expression in Eq. (117) is amended to read

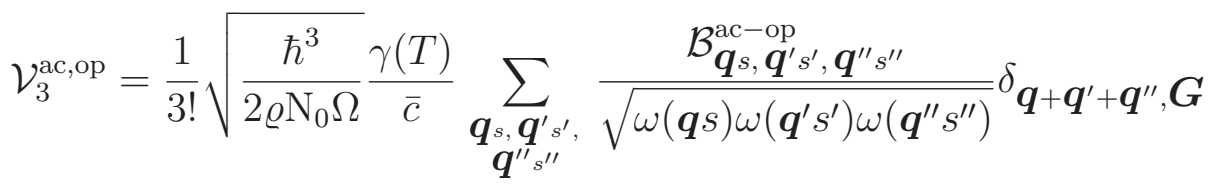

$$
\begin{aligned}
& \times\left(a_{\boldsymbol{q}}^{\dagger}-a_{-} \boldsymbol{q}_{s}\right)\left(a_{-\boldsymbol{q}^{\prime} s^{\prime}}^{\dagger}-a_{\boldsymbol{q}^{\prime} s^{\prime}}\right)\left(a_{\boldsymbol{q}^{\prime \prime} s^{\prime \prime}}^{\dagger}-a_{-} \boldsymbol{q}^{\prime} s^{\prime}\right),
\end{aligned}
$$

with

$$
\begin{aligned}
\mathcal{B}_{i, j, k}^{\text {ac,op }}=[ & \sqrt{\omega(i) \omega(j)}(\omega(i)+\omega(j))\left|\omega_{\Gamma}(k)-\omega(k)\right| \\
& + \text { similar terms with } i, j \text { and } k \text { interchanged }] / 6
\end{aligned}
$$

where $\omega_{\Gamma}(s)$ is the frequency of the $s^{\text {th }}$ branch at zone centre and $\bar{c}$ is an branch-average speed obtained from the acoustic branches in the long wavelength limit (the $\boldsymbol{q}$-point closest to the zone centre). Equation (119) turns into Eq. (117) when only acoustic phonon branches are to be considered (i.e. when $\omega=c q$ and $\omega_{\Gamma}=0$ ).

(c) Anharmonic relaxation time:

Application of Fermi's golden rule leads to the following single-mode relaxation time for a phonon mode $\boldsymbol{q} s$ due to the anharmonic three-phonon interactions in a bulk single crystal (see [48] for details):

$$
\begin{aligned}
\left.\tau^{-1}(\boldsymbol{q} s)\right|_{\mathrm{anh}} ^{\mathrm{bulk}}= & \frac{\pi \hbar \gamma^{2}(T)}{\varrho N_{0} \Omega \bar{c}^{2}} \sum_{\boldsymbol{q}^{\prime} s^{\prime}, \boldsymbol{q}^{\prime \prime} s^{\prime \prime}, \boldsymbol{G}} \frac{\left(\mathcal{B}_{\boldsymbol{q}_{s}, \boldsymbol{q}^{\prime} s^{\prime}, \boldsymbol{q}^{\prime \prime} s^{\prime \prime}}\right)^{2}}{\omega(\boldsymbol{q} s) \omega\left(\boldsymbol{q}^{\prime} s^{\prime}\right) \omega\left(\boldsymbol{q}^{\prime \prime} s^{\prime \prime}\right)} \\
& \times\left[\frac{\bar{n} \boldsymbol{q}^{\prime} s^{\prime}\left(\bar{n}_{\boldsymbol{q}^{\prime \prime} s^{\prime \prime}}+1\right)}{\left(\bar{n} \boldsymbol{q}_{s}+1\right)} \delta\left(\omega(\boldsymbol{q} s)+\omega\left(\boldsymbol{q}^{\prime} s^{\prime}\right)-\omega\left(\boldsymbol{q}^{\prime \prime} s^{\prime \prime}\right)\right) \delta_{\boldsymbol{q}+\boldsymbol{q}^{\prime}, \boldsymbol{q}^{\prime \prime}+\boldsymbol{G}}\right. \\
& \left.+\frac{1}{2} \frac{\bar{n} \boldsymbol{q}^{\prime} s^{\prime} \bar{n}_{\boldsymbol{q}^{\prime \prime} s^{\prime \prime}}}{\bar{n} \boldsymbol{q}_{s}} \delta\left(\omega(\boldsymbol{q} s)-\omega\left(\boldsymbol{q}^{\prime} s^{\prime}\right)-\omega\left(\boldsymbol{q}^{\prime \prime} s^{\prime \prime}\right)\right) \delta_{\boldsymbol{q}+\boldsymbol{G}, \boldsymbol{q}^{\prime}+\boldsymbol{q}^{\prime \prime}}\right], \quad
\end{aligned}
$$

where $\mathcal{B}$ is taken as either $\mathcal{B}^{\text {ac }}$ or $\mathcal{B}^{\text {ac,op }}$.

The processes described by the first and second terms in Eq. (121) may be referred to as Class 1 and Class 2 events, governed by the momentum and energy conservation conditions:

$$
\begin{array}{lll}
\text { Class } 1 \text { events : } & \boldsymbol{q}+\boldsymbol{q}^{\prime}=\boldsymbol{q}^{\prime \prime}+\boldsymbol{G} ; & \omega+\omega^{\prime}=\omega^{\prime \prime}, \\
\text { Class } 2 \text { events : } & \boldsymbol{q}+\boldsymbol{G}=\boldsymbol{q}^{\prime}+\boldsymbol{q}^{\prime \prime} ; & \omega=\omega^{\prime}+\omega^{\prime \prime} .
\end{array}
$$

For each class, an event is called Normal (N) if it involves wavevectors of all participating phonons within the central Brillouin zone. If a reciprocal lattice vector $\boldsymbol{G}$ is required to meet the momentum conservation condition, the event is called Umklapp (U). These processes are schematically illustrated in Fig. 11 for a class 1 event. 


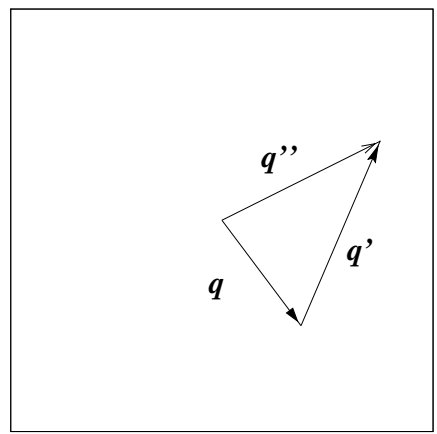

(a)

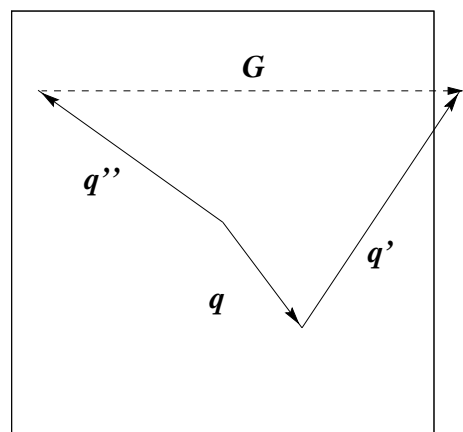

(b)

Figure 11. Schematic illustration of Class 1 three-phonon interactions: (a) an N process $\boldsymbol{q}+\boldsymbol{q}^{\prime}=\boldsymbol{q}^{\prime \prime}$ and (b) an U process $\boldsymbol{q}+\boldsymbol{q}^{\prime}=\boldsymbol{q}^{\prime \prime}+\boldsymbol{G}$.

(d) Numerical evaluation of anharmonic relaxation time:

As discussed earlier for the conductivity expression, numerical evaluation of the anharmonic phonon relaxation time can be performed either by adopting the "special $\boldsymbol{q}$-points' method or a Debye continuum scheme.

\section{d-1 Special $\boldsymbol{q}$-points method:}

The expression in Eq. (121) can be evaluated using the 'special $\boldsymbol{q}$-points' method discussed in Eq. (62). Within that scheme, the momentum conservation conditions in Eqs. (122) and (123) can be approximated as follows

$$
\boldsymbol{q}+\boldsymbol{q}^{\prime} \pm \boldsymbol{q}^{\prime \prime}-\boldsymbol{G}=0 \rightarrow\left|q_{\mu}+q_{\mu}^{\prime} \pm q_{\mu}^{\prime \prime}-G_{\mu}\right| \leq \Delta_{\mu}, \mu=x, y, z
$$

with $\Delta_{\mu}$ being the absolute value of the smallest momentum division of the 'special $\boldsymbol{q}$-points' grid in the $\mu$ direction.

\section{d-2 Debye's isotropic continuum method:}

For a cubic system, application of Debye's elastic isotropic continuum model results in the following expression for the anharmonic phonon relaxation time arising from processes of types $a c+a c \rightleftharpoons a c$ (see [48] for details):

$$
\begin{aligned}
\tau_{\boldsymbol{q}_{s}}^{-1}(3 \mathrm{ph})= & \frac{\hbar q_{\mathrm{D}}^{5} \gamma^{2}(T)}{4 \pi \varrho \bar{c}^{2}} \sum_{s^{\prime} s^{\prime \prime} \epsilon} c_{s} c_{s^{\prime}} \\
& {\left[\int d x^{\prime} x^{\prime 2} x_{+}^{\prime \prime}\left\{1-\epsilon+\epsilon\left(C x+D x^{\prime}\right)\right\} \frac{\bar{n}_{\boldsymbol{q}^{\prime} s^{\prime}}\left(\bar{n}_{+}^{\prime \prime}+1\right)}{\left(\bar{n}_{\boldsymbol{q}_{s}}+1\right)}\right.} \\
& \left.+\frac{1}{2} \int d x^{\prime} x^{\prime 2} x_{-}^{\prime \prime}\left\{1-\epsilon+\epsilon\left(C x-D x^{\prime}\right)\right\} \frac{\bar{n}_{\boldsymbol{q}^{\prime} s^{\prime}} \bar{n}_{-}^{\prime \prime}}{\bar{n}_{\boldsymbol{q}_{s}}}\right] .
\end{aligned}
$$

Here $\bar{n}_{ \pm}^{\prime \prime}=\bar{n}\left(x_{ \pm}^{\prime \prime}\right), x^{\prime}=q^{\prime} / q_{\mathrm{D}}, x_{ \pm}^{\prime \prime}=C x \pm D x^{\prime}$ and $C=c_{s} / c_{s^{\prime \prime}}, D=c_{s^{\prime}} / c_{s^{\prime \prime}}$, $\epsilon=1$ for momentum conserving (normal, or N) processes, and $\epsilon=-1$ for momentum non-conserving (Umklapp, or U) processes. The first and second terms in the above equation are contributed by class 1 events $\boldsymbol{q} s+\boldsymbol{q}^{\prime} s^{\prime} \rightarrow \boldsymbol{q}^{\prime \prime} s^{\prime \prime}$ and class 2 events 
$\boldsymbol{q} s \rightarrow \boldsymbol{q}^{\prime} s^{\prime}+\boldsymbol{q}^{\prime \prime} s^{\prime \prime}$, respectively. The integration limits on the variable $x^{\prime}$, imposed by the energy and momentum conservation conditions in Eqs. (122) and (123), can be derived straightforwardly and are given below.

Class 1 events:

$$
\begin{aligned}
& 0 \quad \leq x \leq 1 \\
& 0, \frac{(1-C) x}{(1+D)} \quad \leq x^{\prime} \leq \frac{(1+C) x}{(1-D)}, \frac{(1-C x)}{D}, 1 \quad N \text { processes, } \\
& 0, \frac{(2-(1+C) x)}{(1+D)} \leq x^{\prime} \leq \frac{(1-C x)}{D}, 1 \quad U \text { processes. }
\end{aligned}
$$

Class 2 events:

$N$ processes:

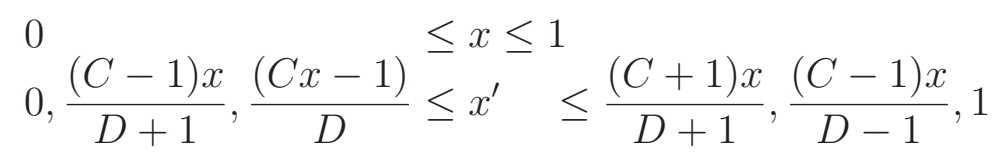

$U$ processes:

$$
\begin{array}{ll}
\frac{2}{1+C} & \leq x \leq 1 \\
0, \frac{2-(1+C) x}{1-D}, \frac{C x-1}{D}, \frac{(C+1) x-2}{D+1} & \leq x^{\prime} \leq \frac{(C+1) x-2}{D-1}, 1 .
\end{array}
$$

4.4.2. Nanostructures and Nanocomposite structures: For nanostructures an appropriate effective boundary length will have to be determined. For example, when considering heat transport across a thin film, such a length will be determined by the film thickness. The expression for the mass-defect scattering strength in Eq. (108) can be used for a superlattice structure $(\mathrm{AB})_{n}(\mathrm{CD})_{m}$ by considering it as a compound with formula $\mathrm{A}_{n} \mathrm{~B}_{n} \mathrm{C}_{m} \mathrm{D}_{m}$. In nanocomposite materials, two additional forms of scattering would need to be considered: interface scattering (from mass-mixing across interfaces and interface defects such as broken bonds and dislocations) and mini-Umklapp anharmonic scattering due to the presence of more than one material per large unit cell (LUC).

\section{(i) Anharmonic scattering in nanocomposites:}

The form of the cubic anharmonicity for a single crystal, such as that described either in Eq. (117) or in Eq. (119), must be modified when dealing with a periodically produced composite structure. Consider a superlattice $\mathrm{A}_{n_{1}} \mathrm{~B}_{n_{2}}$ whose every unit cell contains $n_{1}$ atomic layers of material A with atomic mass $M_{A}$ and $n_{2}$ atomic layers of material B with atomic mass $M_{B}$. Obviously the periodicity along the superlattice growth direction will be larger than the bulk periodicity in either of the two bulk materials A or B. Atomic vibrations within each unit cell must be phase correlated. Employing a simple one-dimensional approach, Ren and Dow [126] suggested that the three-phonon matrix element in such a structure will be proportional to the fractional 
mass "disorder" $\Delta M / M_{0}$ in the SL, where

$$
\frac{1}{M_{0}}=\frac{1}{2}\left[\frac{1}{M_{a}}+\frac{1}{M_{B}}\right], \quad \Delta\left(\frac{1}{M}\right)=\frac{1}{2}\left[\frac{1}{M_{a}}-\frac{1}{M_{B}}\right] .
$$

Due to superlattice unit cells being larger than bulk unit cells, some bulk threephonon Normal (N) processes turn into "mini-Umklapp" processes, with the shortest superlattice $\boldsymbol{G}_{\mathrm{SL}}$ vector being shorter than the shortest $\boldsymbol{G}_{\text {bulk }}$ vector in either of the bulk materials. Such three-phonon processes in the superlattice structure have been termed 'mini-Umklapp processes'. As the three-phonon Umklapp scattering rate is proportional to the square of the reciprocal lattice vector $\boldsymbol{G}[35]$, the scattering rate of the miniUmklapp processes in the SL structure will be proportional to $\left(\Delta M / M_{0}\right)^{2} /\left(n_{1}+n_{2}\right)^{2}$ [126].

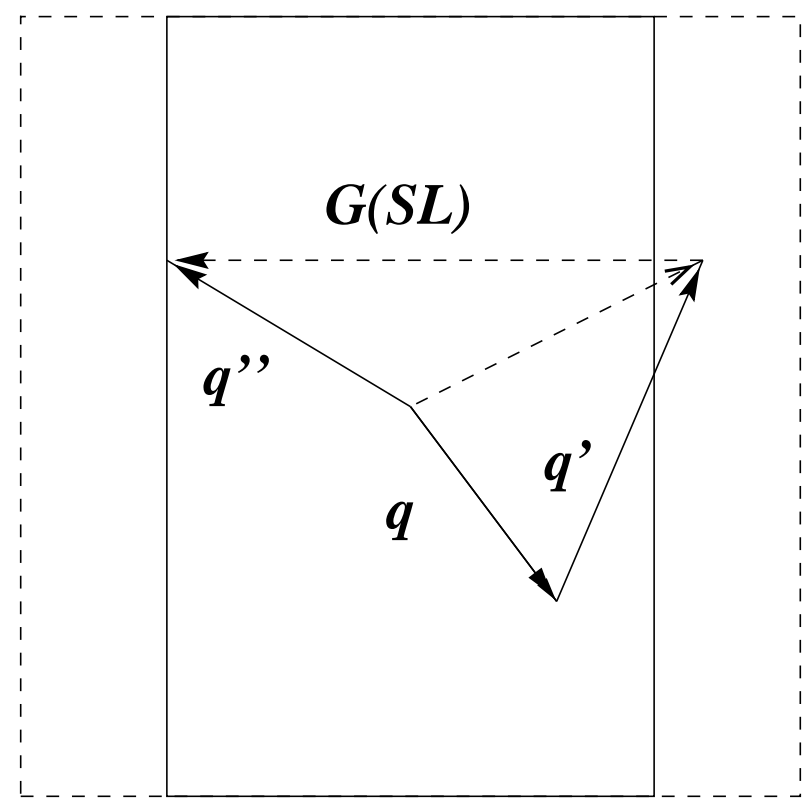

Figure 12. Schematic illustration of how a three-phonon Normal (N) process $\left(\boldsymbol{q}+\boldsymbol{q}^{\prime}=\boldsymbol{q}^{\prime \prime}\right)$ in a bulk structure turns into a mini-Umklapp process $\left(\boldsymbol{q}+\boldsymbol{q}^{\prime}=\boldsymbol{q}^{\prime \prime}+\boldsymbol{G}_{\mathrm{SL}}\right)$ in a superlattice structure. The solid and dashed rectangles indicate the superlattice and bulk Brillouin zones, respectively.

Following the considerations made in the work by Ren and Dow [126], and employing the cubic anharmonic potential presented in Eq. (119), Srivastava and coworkers [89, 90, 127, 128] have developed the following expression for three-phonon scattering rate in a $\mathrm{A}_{n} \mathrm{~B}_{n}$ superlattice structure

$$
\begin{aligned}
\left.\tau^{-1}(\boldsymbol{q} s)\right|_{\mathrm{anh}} ^{\mathrm{SL}}= & \frac{\pi \hbar \varrho^{2} \gamma^{2}(T)}{N_{0} \Omega \bar{c}^{2}} \sum_{\boldsymbol{q}^{\prime} s^{\prime}, \boldsymbol{q}^{\prime \prime} s^{\prime \prime}, \boldsymbol{G}} \frac{\left(\mathcal{B}_{\boldsymbol{q}_{s}, \boldsymbol{q}^{\prime} s^{\prime}, \boldsymbol{q}^{\prime \prime} s^{\prime \prime}}\right)^{2} \mathcal{D}\left(\boldsymbol{q} s, \boldsymbol{q}^{\prime} s^{\prime}, \boldsymbol{q}^{\prime \prime} s^{\prime \prime}\right)}{\omega(\boldsymbol{q} s) \omega\left(\boldsymbol{q}^{\prime} s^{\prime}\right) \omega\left(\boldsymbol{q}^{\prime \prime} s^{\prime \prime}\right)} \\
& \times\left[\frac{\bar{n} \boldsymbol{q}^{\prime} s^{\prime}\left(\bar{n} \boldsymbol{q}^{\prime \prime} s^{\prime \prime}+1\right)}{\left(\bar{n} \boldsymbol{q}_{s}+1\right)} \delta\left(\omega(\boldsymbol{q} s)+\omega\left(\boldsymbol{q}^{\prime} s^{\prime}\right)-\omega\left(\boldsymbol{q}^{\prime \prime} s^{\prime \prime}\right)\right) \delta_{\boldsymbol{q}+\boldsymbol{q}^{\prime}, \boldsymbol{q}^{\prime \prime}+\boldsymbol{G}}\right. \\
& \left.+\frac{1}{2} \frac{\bar{n} \boldsymbol{q}^{\prime} s^{\prime} \bar{n}_{\boldsymbol{q}^{\prime \prime} s^{\prime \prime}}}{\bar{n}_{\boldsymbol{q}_{s}}} \delta\left(\omega(\boldsymbol{q} s)-\omega\left(\boldsymbol{q}^{\prime} s^{\prime}\right)-\omega\left(\boldsymbol{q}^{\prime \prime} s^{\prime \prime}\right)\right) \delta_{\boldsymbol{q}} \boldsymbol{G}, \boldsymbol{q}^{\prime}+\boldsymbol{q}^{\prime \prime}\right]
\end{aligned}
$$


where $\varrho$ is the average mass density in the SL structure. The factor $\mathcal{D}\left(\boldsymbol{q} s, \boldsymbol{q}^{\prime} s^{\prime}, \boldsymbol{q}^{\prime \prime} s^{\prime \prime}\right)$, which may be called the "dual mass term", arises from the composite nature of the superlattice (i.e. due to the presence of materals A and B). This term is interlinked with the onset of the mini-Umklapp anharmonic processes and the relative amplitudes of the atomic vibrations in the materials $\mathrm{A}$ and $\mathrm{B}$ within each unit cell. The following simple analytical expression for this term can be obtained by considering the diatomic linear chain model along the SL growth direction

$$
\mathcal{D}\left(\boldsymbol{q} s, \boldsymbol{q}^{\prime} s^{\prime}, \boldsymbol{q}^{\prime \prime} s^{\prime \prime}\right)=\frac{1}{64}\left(\frac{\mathcal{A}_{A B}}{2 \varrho_{A}^{3 / 2}}+\frac{\mathcal{A}_{B A}}{2 \varrho_{B}^{3 / 2}}\right)^{2}
$$

with

$$
\mathcal{A}_{i j}=1+\frac{\varrho_{i}^{1 / 2}}{\varrho_{j}^{1 / 2}}\left(\frac{e_{j}}{e_{i}}+\frac{e_{j}^{\prime}}{e_{i}^{\prime}}+\frac{e_{j}^{\prime \prime}}{e_{i}^{\prime \prime}}\right)+\frac{\varrho_{i}}{\varrho_{j}}\left(\frac{e_{j} e_{j}^{\prime}}{e_{i} e_{i}^{\prime}}+\frac{e_{j}^{\prime} e_{j}^{\prime \prime}}{e_{i}^{\prime} e_{i}^{\prime \prime}}+\frac{e_{j} e_{j}^{\prime \prime}}{e_{i} e_{i}^{\prime \prime}}\right)+\frac{\varrho_{i}^{3 / 2}}{\varrho_{j}^{3 / 2}}\left(\frac{e_{j} e_{j}^{\prime} e_{j}^{\prime \prime}}{e_{i} e_{i}^{\prime} e_{i}^{\prime \prime}}\right),
$$

where $\varrho_{i}$ is the material density, and $e_{i} \equiv e_{i}(\boldsymbol{q} s), e_{i}^{\prime} \equiv e_{i}\left(\boldsymbol{q}^{\prime} s^{\prime}\right), e_{i}^{\prime \prime} \equiv e_{i}\left(\boldsymbol{q}^{\prime \prime} s^{\prime \prime}\right)$ are the vibrational eigenvectors in the $i^{\text {th }}$ segment: $i=A(B)$. The ratio of the amplitudes can be expressed as

$$
\frac{e_{B}}{e_{A}}=\frac{\left[\frac{1}{M_{0}}-\Delta\left(\frac{1}{M}\right)\right] \cos \left(l_{z} q_{z}\right)}{\left[\left(\frac{1}{M_{0}}\right)^{2}+\left\{\Delta\left(\frac{1}{M}\right)\right\}^{2} \sin ^{2}\left(l_{z} q_{z}\right)\right]^{1 / 2}-\Delta\left(\frac{1}{M}\right)}
$$

where $M_{0}=\left(M_{A}^{-1}+M_{B}^{-1}\right) / 2$ and $\Delta(1 / M)=\left(M_{A}^{-1}-M_{B}^{-1}\right) / 2$, and $l_{z}$ is the SL period along the $z$ direction.

\section{(ii) Interface scattering in superlattices:}

Realistic superlattices are likely to be characterised by three types of interface defects: (i) mass mixing or smudging due to diffusion or mixing of atoms across interfaces, (ii) epitaxial layer roughening and (iii) misfit dislocation or missing bonds at interfaces. Such defects produce additional phonon scattering mechanisms. Some effort has recently been made to model interface scattering of phonons, notably in the works of Landry and McGaughy [129], Tian et al. [130], Hepplestone and Srivastava [127], Thomas and Srivastava [89, 90]. Landry and McGaughey [129] included interfacial species mixing in a superlattice sample by randomly assigning the species of each atom in the interface region according to the distribution

$$
x(z)=x_{L}+\frac{1}{2}\left(x_{R}-x_{L}\right)\left[1+\tanh \left(\frac{4 z}{D}\right)\right],
$$

where $D$ is the thickness of the mass-mixing region, $\mathrm{z}$ is measured relative to the closest interface, and $x_{L}$ and $x_{R}$ are the desired unmixed concentrations on the left and right sides of the interface. Tian et al. [130] assigned the occupancy in the interface region of atomic masses from the two sides according to some probability (uniform or Gaussian), constrained by the thickness of the rough region. Srivastava's group [89, 90, 127] attempted to frame a theory of interface mass mixing slightly more rigorously. Here we will briefly discuss the approach described in Refs. [89, 90, 127]. 
(a) Perturbed Hamiltonian resulting from interface mass mixing:

Consider a periodic un-reconstructed superlattice $\mathrm{A}_{n_{1}} \mathrm{~B}_{n_{2}}$ with $N_{0}$ unit cells. Assuming that interface mass mixing takes place within $J$ layers on either side of an interface, the perturbed defect Hamiltonian can be expressed as

$$
H^{\prime}(\mathrm{IMS})=\frac{1}{2} \sum_{i=n_{1}-J}^{n_{1}}\left(M_{i}\left|\mathbf{v}_{\mathbf{i}}\right|^{2}-M_{A}\left|\mathbf{v}_{\mathbf{A}}\right|^{2}\right)+\frac{1}{2} \sum_{i=n_{1}+1}^{n_{1}+J}\left(M_{i}\left|\mathbf{v}_{\mathbf{i}}\right|^{2}-M_{B}\left|\mathbf{v}_{\mathbf{B}}\right|^{2}\right),
$$

where $M_{i}$ is the mass of the $i^{\text {th }}$ atom, and $\mathbf{v}_{i}=\mathrm{d} \mathbf{u}_{\mathbf{i}} / \mathrm{d} t$ with $\mathbf{u}_{\mathbf{i}}$ being the displacement of the $i^{\text {th }}$ atom relative to its neighbours. Generally it is very difficult to judge a value for the number $J$ of atomic layers affected, and the amount and distribution of mixed masses in such layers.

(b) Perturbed Hamiltonian resulting from interface dislocations due to broken bonds:

Superlattices grown using lattice-mismatched atomic layers are known to be characterised by the presence of misfit dislocations at interfaces. Consider an interface dislocation as a series of randomly missing bonds located near the interface within a SL unit cell. With such a consideration we can write the perturbed superlattice Hamiltonian as

$$
H^{\prime}(\mathrm{IDS})=\frac{1}{2} \sum_{i=n_{1}-I}^{n_{1}}\left(K_{0}\left|\boldsymbol{u}_{i}\right|^{2}-K_{A}\left|\boldsymbol{u}_{A}\right|^{2}\right)+\frac{1}{2} \sum_{i=n_{1}+1}^{n_{1}+I}\left(K_{0}\left|\boldsymbol{u}_{i}\right|^{2}-K_{B}\left|\boldsymbol{u}_{B}\right|^{2}\right),
$$

where $u_{i}$ is the relative displacement between two neighbouring atoms and $I$ is the number of atomic layers with broken bonds on either side of an interface, $K_{A}\left(K_{B}\right)$ represents the interatomic spring constant in the layer $\mathrm{A}(\mathrm{B})$, and $K_{0}$ represents a spring constant in the dislocation region, i.e. has a value equal or close to zero, for broken or missing bonds.

\section{(c) IMS relaxation rate:}

With a reasonable choice of interface mass mixing parameters, Fermi's golden rule can be applied to derive an expression for the phonon scattering rate.

Consider $\alpha_{j}$ as the fraction of the $j^{\text {th }}$ interface layer being mass-mixed. Noting that one layer represents the $\left[\frac{1}{\left(n_{1}+n_{2}\right)}\right]^{\text {th }}$ fraction of the SL period and $\alpha_{j} /\left(n_{1}+n_{2}\right)$ is the occupancy of the $j^{\text {th }}$ layer with mass difference $\Delta M_{j}$, Hepplestone and Srivastava [127] derived the following result:

$$
\begin{aligned}
\tau_{\mathrm{IMS}}^{-1}(\boldsymbol{q} s)= & \frac{\pi F_{\mathrm{IMS}}}{2 N_{0}\left(n_{1}+n_{2}\right)^{2}} \int d \omega\left(\boldsymbol{q}^{\prime} s^{\prime}\right) g\left(\omega\left(\boldsymbol{q}^{\prime} s^{\prime}\right)\right) \omega(\boldsymbol{q} s) \omega\left(\boldsymbol{q}^{\prime} s^{\prime}\right) \\
& \times \frac{\bar{n}\left(\boldsymbol{q}^{\prime} s^{\prime}\right)+1}{\bar{n}(\boldsymbol{q} s)+1} \delta\left(\omega(\boldsymbol{q} s)-\omega\left(\boldsymbol{q}^{\prime} s^{\prime}\right)\left[\left(1-\frac{e_{A} e_{A}^{\prime}}{e_{B} e_{B}^{\prime}}\right)^{2}+\left(1-\frac{e_{B} e_{B}^{\prime}}{e_{A} e_{A}^{\prime}}\right)^{2}\right],\right.
\end{aligned}
$$

where $F_{\mathrm{IMS}}=\sum_{j} \alpha_{j}^{2}$ is regarded as a parameter related to the amount of mixing at the interface, and other terms have been introduced earlier. The factor $1 /\left(n_{1}+n_{2}\right)^{2}$ in Eq. (137) implies that the strength of the IMS rate is greatest for thin SLs and decreases as the SL becomes thicker. This correctly ensures that the IMS mechanism would not exist in bulk systems (viwed as infinitely thick superlattices). More recently, Thomas and 
Srivastava [90] have presented a more detailed and refined treatment of the probability of mass mixing across superlattice interfaces.

\section{(d) IDS relaxation rate:}

With a reasonable choice of broken bonds and changes in interface force constants, Fermi's golden rule can be applied to derive an expression for the phonon scattering rate. On the basis of several simplifying assumptions and approximations, Hepplestone and Srivastava [127] derived the following result:

$$
\begin{aligned}
\tau_{\mathrm{IDS}}^{-1}(\boldsymbol{q} s)= & \frac{\pi \omega_{0}^{4}}{4 N_{0}} \frac{F_{\mathrm{IDS}}}{\left(n_{1}+n_{2}\right)^{2}} \int \mathrm{d} \omega\left(\boldsymbol{q}^{\prime} s^{\prime}\right) \frac{g\left(\omega\left(\boldsymbol{q}^{\prime} s^{\prime}\right)\right)}{\omega(\boldsymbol{q} s) \omega\left(\boldsymbol{q}^{\prime} s^{\prime}\right)} \frac{\bar{n}\left(\boldsymbol{q}^{\prime} s^{\prime}\right)+1}{\bar{n}(\boldsymbol{q} s)+1} \\
& \times \delta\left(\omega(\boldsymbol{q} s)-\omega\left(\boldsymbol{q}^{\prime} s^{\prime}\right)\right)\left[1+\left(\frac{e_{A} e_{A}^{\prime}}{e_{B} e_{B}^{\prime}}\right)^{2}+1+\left(\frac{e_{B} e_{B}^{\prime}}{e_{A} e_{A}^{\prime}}\right)^{2}\right]
\end{aligned}
$$

where $F_{\text {IDS }}$ is a measure of dislocation concentration and $\omega_{0}$ can be approximated as the highest zone-centre frequency. The factor $1 /\left(n_{1}+n_{2}\right)^{2}$ in Eq. (138) implies that the strength of the IDS rate is greatest for thin SLs and decreases as the superlattice becomes thicker. This correctly ensures that IDS mechanism would not exist in bulk systems (viewed as infinitely thick SLs).

(iii) Phonon scattering from polydispersed nanowires and nanodots in a host matrix:

The IMS and IDS mechanisms discussed in the preceding sub-section for superlattice structures can potentially be extended to superlattice structures made of periodically dispersed nanowires of rectangular cross-section and nanodots of planar faces. Different formalisms have been proposed to derive the phonon scattering rate from nanodots of spherical shape embedded in an isotropic solid medium [131, 132, 133, 134]. These rely on the embedding material (i.e. nanodots) being considered as a small perturbation to the phonons in the host matrix. Ying and Truell [131] employed the acoustic Mie theory to derive the phonon scattering rate from spherical particles in solids. Kim and Majumdar [132] proposed an approximate analytical solution to estimate the phonon scattering rate from polydispersed spherical nanoparticles in a host medium. Expressing a size parameter $\chi$ as $\chi=q R$, where $q$ is the phonon wavenumber and $R$ is the particle size, Their work considered two limiting cases for the nanoparticle size: $\chi<<1$ and $\chi \rightarrow \infty$. For particle sizes much smaller than the phonon wavelength (i.e. $\quad \chi<<1$ ) they applied the first-order time-dependent perturbation theory by considering the mass difference, as well as the force constant difference, between the spherical nanoparticle and its host medium. Their expression for the phonon relaxation rate is similar in its Rayleigh scattering form to Eq. (107) but differs in the details of the multiplicative factor. In the geometrical scattering limit, when $\chi \rightarrow \infty$, they used the van de Hulst approximation for anomalous diffraction to estimate the scattering cross section as a function of the acoustic impedance mismatch between the host medium and the spherical nanoparticle. 
Minnich and Chen [133] introduced a modified effective medium formulation for composites where the characteristic length of the nanoparticle (or nanodot (ND)) is of the order of or smaller than the phonon mean free path. In this formulation the phonon mean free paths of the host and particle phases are expressed as

$$
\frac{1}{\Lambda_{\text {effective host }}}=\frac{1}{\Lambda_{\text {bulk host }}}+\frac{1}{\Lambda_{\text {coll }}} ; \quad \frac{1}{\Lambda_{\text {effective ND }}}=\frac{1}{\Lambda_{\text {bulk-ND }}}+\frac{1}{d},
$$

where $d$ is the diameter of spherical nanoparticles and $\Lambda_{\text {coll }}$ is a measure of the distance travelled divided by the number of collisions.

The modified effective medium formulation was further improved by Behrang et al. [135] who incorporated a new consideration of the nanodot-host interface (i.e. of $\left.\Lambda_{\text {coll }}\right)$ and the influence of various degrees of agglomeration of the dispersed particles. Two types of particle-host interactions were considered: collision in which the incoming and outcoming phonons remain on the same side of the interface, and transmission interactions in which phonons pass through the interface. Each of these interactions is again of two types: diffuse in which the direction of the phonon after the interactions is independent of the direction of the impacting phonons, and specular in which the phonon incident angle influences the outcome of the interaction. As indicated earlier, the degree of specularity generally decreases with the roughness of the interface. The phonon mean free paths in the nanodot and the host are then expressed as

$$
\frac{1}{\Lambda_{j}^{(i)}}=\frac{1}{\Lambda_{\mathrm{bulk}, j}^{(i)}}+\frac{1}{\Lambda_{\mathrm{bulk}, j}^{(i)}}+\frac{1}{\Lambda_{\mathrm{collision}, j}^{(i)}}+\frac{1}{\Lambda_{\mathrm{TBR}, j}^{(i)}} ; \quad i=\text { spec, diff; } \quad j=\text { host, ND, }
$$

where $\Lambda_{\mathrm{TBR}}$ is the thermal boundary resistance mean free path (a ratio between the transmission length and the number of transmissions). We can finally express $\Lambda=p \Lambda^{\text {(spec) }}+(1-p) \Lambda^{\text {(diff) }}$, with $p$ as a phenomenological specularity parameter, $0 \leq p \leq 1$. The expressions for $\Lambda_{\mathrm{TBR}}^{\text {(diff, spec) }}$ include the probabilities of transmission $t_{j l}^{\text {diff,spec }}$, which are given as follows

$$
\begin{aligned}
t_{j l}^{\mathrm{diff}} & =\frac{C_{l} v_{l}}{C_{j} v_{j}+C_{l} v_{l}}, \quad j, l=\mathrm{ND}, \text { host; } j \neq l \\
t_{\mathrm{ND} \text { host }}^{\mathrm{spec}} & =\frac{4 \varrho_{\mathrm{ND}} v_{\mathrm{ND}} \varrho_{\mathrm{host}} v_{\mathrm{host}} \mu_{\mathrm{ND}} \mu_{\mathrm{host}}}{\left(\varrho_{\mathrm{ND}} v_{\mathrm{ND}} \mu_{\mathrm{ND}}+\varrho_{\mathrm{host}} v_{\mathrm{host}} \mu_{\mathrm{host}}\right)^{2}} \\
t_{\text {host ND }}^{\mathrm{spec}} & =\frac{C_{\mathrm{ND}} v_{\mathrm{ND}}^{3}}{C_{\mathrm{host}} v_{\mathrm{host}}^{3}} t_{\mathrm{ND} \text { host }}^{\mathrm{spec}},
\end{aligned}
$$

where $C$ is specific heat, $v$ is the acoustic phonon speed, $\varrho$ is the mass density of the host material, and $\mu_{\text {host }}=\cos \theta_{\text {host }}$ and $\mu_{\mathrm{ND}}=\cos \theta_{\mathrm{ND}}$ are related by Snell's law $\sin \theta_{\text {host }} / \sin \theta_{\mathrm{ND}}=v_{\text {host }} / v_{\mathrm{ND}}$.

(iv) Obscure variables for phonon scatterings in nanostructures and nanocomposites As can be appreciated from the discussion presented in this sub-section, phonon scatterings from interface mass-smearing (IMS), interface broken bonds (IDS) and nanodot embedding (thermal boundary or Kapitza resistance) are all characterised with obscure variables and adjustable parameters are required to address these. We 
will briefly return to the issue of the Kapitza resistance in Sect 5.3. Such parameters are inherently dependent on the growth condition and the growth technique employed in preparing such structures or materials. In other words, the parameters are highly variable and generally not known from experiment and are highly uncontrollable. This means that the predictivity of the different interface scattering mechanisms is limited, and while these clearly allow one to explain existing thermal conductivity results with the use of adjustable or fitting parameters, more physically appealing schemes with greater applicability remain a want.

\section{Phonon Conductivity Results for Bulk and Nanomaterials}

In order to appreciate the general effect of nanostructuring on phonon transport, it is essential to make a systematic presentation of the lattice thermal conductivity of relevant single crystal bulk materials, single crystal alloys, and nanostructures. We will do so by considering just a few systems.

\subsection{Bulk materials}

Figure 13 shows the calculated lattice thermal conductivity of bulk Si and Ge using the iterative solution of the phonon transport equation discussed in Sec. 4.3.2(iii) and the third-order force constants obtained ab initio from the application of DFPT and the planewave pseudopotential method [120]. It is gratifying to note that there is very good agreement between the calculated results and experimental measurements in the temperature range 100-300 K.

Figure 14 shows the lattice thermal conductivity of bulk Si and Ge for a much larger temperature range. Theoretical results obtained in Ref. [122] from the application of the isotropic Callaway model, using Eqs. (102) and (125), and adjustable values of the massdefect parameter and Grüneisen's constant, agree well with experimental measurements. In general, with increase in temperature, $\kappa$ increases as $T^{3}$ at low temperatures when the phonon-boundary scattering dominates, reaches a maximum in the temperature range 10-30 K due to the combined effect of defect and anharmonic scatterings of phonons, and decreases as $1 / T$ at high temperatures when three-phonon scattering processes dominate. The maximum value of $\kappa$ is generally determined by the average atomic mass and the level of sample purity. For Si $\kappa$ assumes a maximum value of approximately $5000 \mathrm{~W} / \mathrm{m}-\mathrm{K}$ at around $20 \mathrm{~K}$. For Ge $\kappa$ assumes a maximum value of approximately $1300 \mathrm{~W} / \mathrm{m}-\mathrm{K}$ at around $20 \mathrm{~K}$. The room temperature values of $\kappa$ are 130 and $58 \mathrm{~W} / \mathrm{m}-\mathrm{K}$ for $\mathrm{Si}$ and Ge, respectively. Si is categorised as a 'high thermal conductivity material' (a material with room-temperature conductivity larger than $100 \mathrm{~W} / \mathrm{m}-\mathrm{K}$ ). 


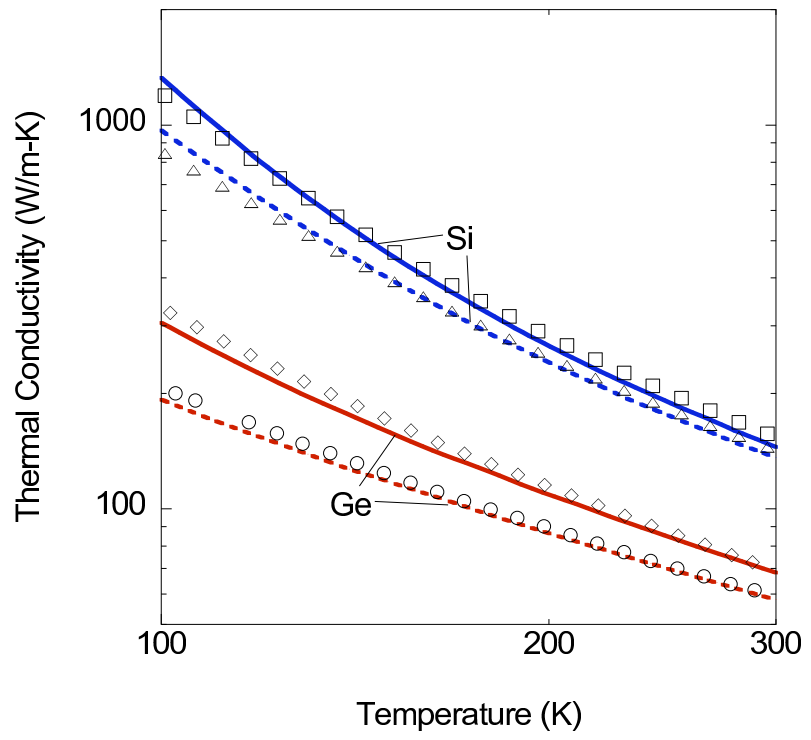

Figure 13. Lattice thermal conductivity of bulk $\mathrm{Si}$ and Ge. Results of ab initio calculations in Ref. [120] of the lattice thermal conductivity. Blue dashed and blue solid curves for natural and isotopically enriched $\mathrm{Si}$, and red dashed and red solid cruves for natural and isotopically enriched Ge. Symbols represent experimental measurements from Refs. [136] and [137]. Reproduced, with authors' permission, from Mingo et al. [120].

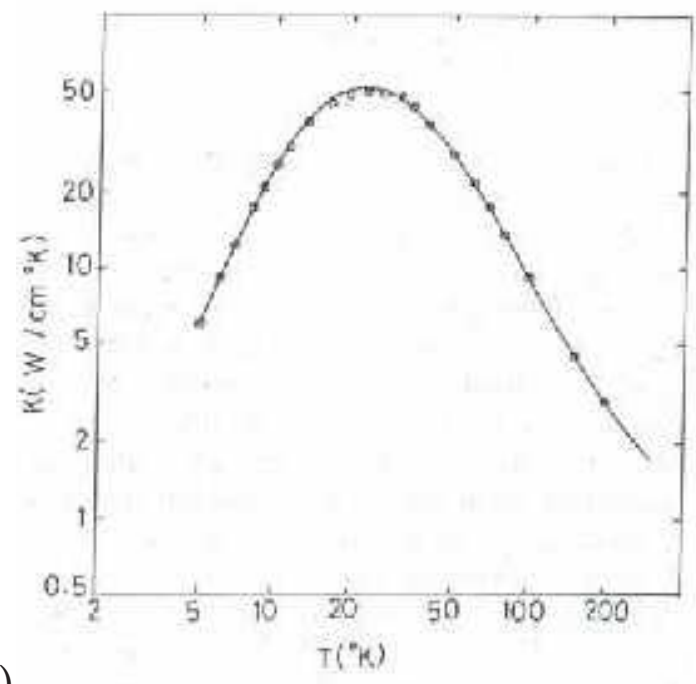

(a)

(b)

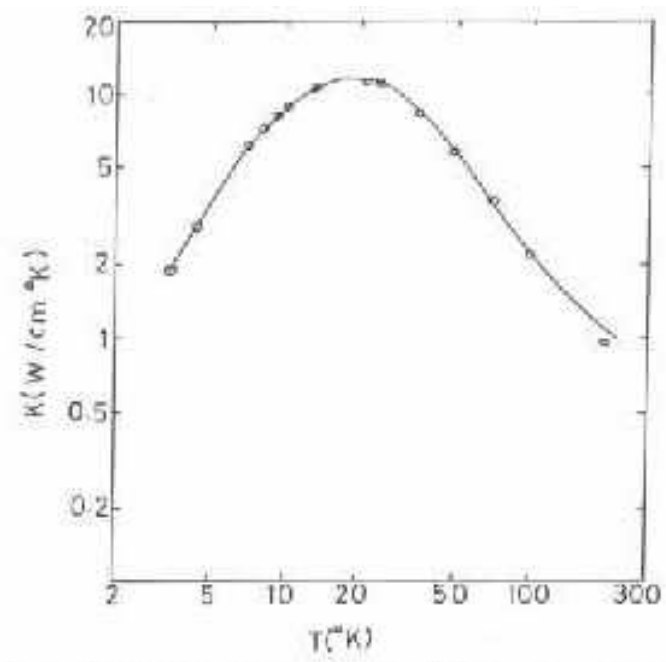

Figure 14. Lattice thermal conductivity of bulk (a) $\mathrm{Si}$ and (b) natural Ge. The solid curves represent results from the isotropic Callaway model, using Eqs. (102) and (125), and adjustable values of the mass-defect parameter and Grüneisen's constant. Symbols represent experimentally measured values: Si [138]; Ge (natural sample of Geballe and Hull [139]). Reproduced, with permission, from Srivastava [122].

\subsection{Single crystal alloys}

Figure 15(a) shows results of lattice thermal conductivity calculations by Vinning [140], based on Callaway's formalism and Debye's isotropic continuum approximation, 
at several temperatures and for several carrier concentrations of n-type $\mathrm{Si}_{0.8} \mathrm{Ge}_{0.2}$ alloys. As expected, the conductivity decreases with increasing temperature and with increasing ionized donor impurity. The calculated room temperature conductivity value of close to $5.8 \mathrm{~W} / \mathrm{m}-\mathrm{K}$ for even for the lowest donor concentration considered in this work $\left(10^{25} \mathrm{~m}_{3}\right)$ is much lower than the result for undoped Ge. Consistent with this work, other calculations, either based on the application of the Debye-Callaway model [141] or a realistic numerical scheme based on a combination of first-principles and phenomenological theories and the single-mode relaxation time approximation [123], have also confirmed experimental measurements that the room temperature phonon conductivity of an n-type $\mathrm{Si}_{0.8} \mathrm{Ge}_{0.2}$ with donor concentration of $7.3 \times 10^{25} \mathrm{~m}^{-3}$ is close to $4.1 \mathrm{~W} / \mathrm{m}-\mathrm{K}$, much lower than that for undoped bulk Ge.

Garg et al. [142] have computed the thermal conductivity of a disordered SiGe alloy from first-principles DFPT within the single mode relaxation time approximation. Their results for $\mathrm{Si}_{0.3} \mathrm{Ge}_{0.7}$ are presented in Fig. 15(b). Surprisingly, even without considering any temperature dependence of the third-order force constants, their approach is found to accurately reproduce available experimental results. Their work makes the most interesting observation that disorder results in an increased anharmonic scattering of phonons through a modification of their vibration eigenmodes.

(a)

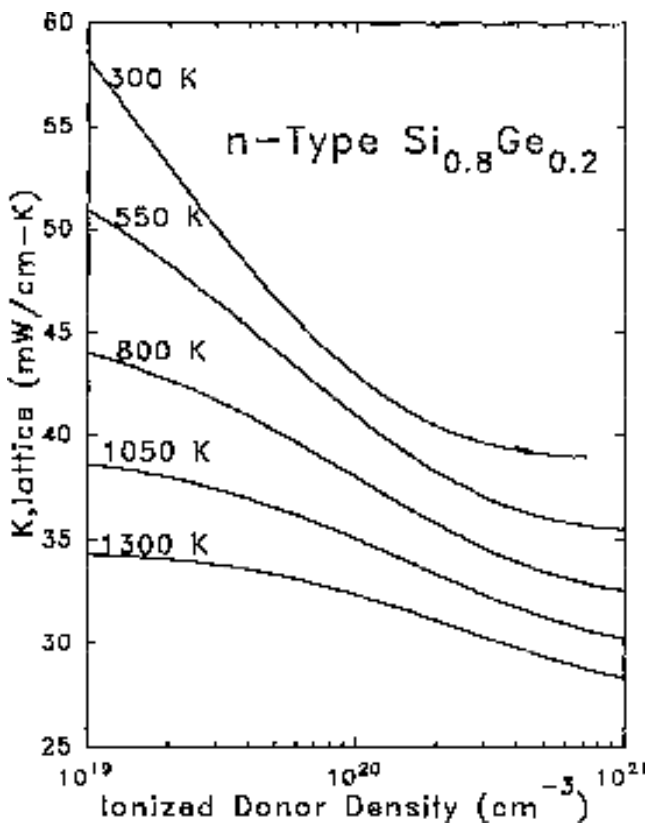

(b)

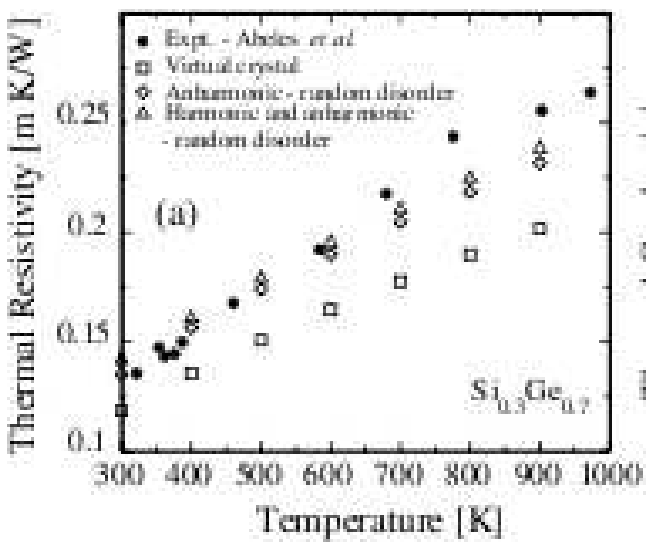

Figure 15. Panel (a): Lattice thermal conductivity of an n-type $\mathrm{Si}_{0.8} \mathrm{Ge}_{0.2}$ alloy doped with several amounts of donor concentrations. Reproduced with permission from citeVining-1991. Copyright (1991), AIP Publishing LLC. Panel (b): Lattice thermal resistivity of disordered $\mathrm{Si}_{0.3} \mathrm{Ge}_{0.7}$. The open squares and the open diamonds represent numerical results obtained by Garg et al. [142] by employing a first-principles DFPT approach. Filled squares show experimental measurements from Ref. [143]. Reproduced, with permission, from Garg et al. [142]. 


\subsection{Nanomaterials}

A full-scale calculation, using a large unit cell suitable for descring a composite system, should be capable of providing all information required for phonon transport through the system. It is, however, convenient to distinguish three regimes, dependent on the relative sizes of the unit cell size for the composite system and the phonon mean free path $\Lambda$ [144]. Let us consider a SL structure of repeat period size $d_{S L}$, containing material A of layer thickness $d_{A}$ and material B of layer thickness $d_{B}$. The three regimes are:

[A] If $d_{S L}>>\Lambda$, the cross-plane conductivity $\kappa_{\perp}$ will just be the weighted average of the bulk conductivities:

$$
\kappa_{\perp}=\frac{1}{d_{A}+d_{B}}\left(\frac{d_{A}}{\kappa_{A}}+\frac{d_{B}}{\kappa_{B}}\right) .
$$

[B] If $d_{S L} \sim \Lambda$, the SL conductivity should be expressed as

$$
\kappa_{\perp}=\frac{1}{d_{A}+d_{B}}\left(\frac{d_{A}}{\kappa_{A}}+\frac{d_{B}}{\kappa_{B}}+\frac{2}{\sigma_{\mathrm{K}}}\right),
$$

where $\sigma_{\mathrm{K}}$ is the Kapitza (boundary, or interface) conductance. This consideration was made by Behrang et al. [135] in Eq. (4.4.2) for expressing the phonon mean free path for polydispersed nanodots in a host matrix.

[C] For the case $d_{S L}<<\Lambda$ a full-scale theory is required, which accounts for three new phenomena: (i) phonon dispersion relations for the superlattice, (ii) onset of SLperiod-related mini-Umklapp processes and (iii) phonon-interface scattering processes.

For case $[\mathrm{B}]$, viz. when $d_{S L} \sim \Lambda$, the interface resistance $\rho_{\mathrm{K}}$ can be evaluated by making three calculations, all with the consideration of a large unit cell that can accommodate all the three systems $\mathrm{X}, \mathrm{Y}$ and $\mathrm{X} / \mathrm{Y}$ : two separate calculations for the two bulks $\mathrm{X}$ and $\mathrm{Y}$ and a third calculation for the composite $\mathrm{X} / \mathrm{Y}$ by considering it as periodic system. However, such an effort would require computational resources that are at present out of reach. This has necessitated the description of the conductivity using Eq. (145), which requires two bulk calculations using small unit cells and the evaluation of $\sigma_{\mathrm{K}}$ as described in Sect. 4.4.2(iii). Two theoretical models [145] are generally used to estimate $\sigma \mathrm{K}$ : the acoustic mismatch model (AMM) and the diffuse mismatch model (DMM). While neither model is capable of making accurate predictions, the AMM and DMM provide reasonable understanding in the limiting cases of perfectly sharp and grossly rough interfaces, respectively. In this review article we will present results for thin-sized nanomaterials that can be placed in the regimes $[\mathrm{B}]$ and $[\mathrm{C}]$.

\section{(i) Ultrathin superlattices:}

Experimental measurements of the lattice thermal conductivity of ultrathin semiconductor superlattices have been made by employing the $3 \omega$ technique [146]. Lee et al. [147] measured the cross-plane thermal conductivity of Si/Ge superlattices with superlattice periods ranging from 30 to $300 \AA$. Borca-Tasciuc et al. [148] performed the cross-plane thermal conductivity measurement on a number of symmetrically strained thin Si/Ge superlattices (one period ranging from $40 \AA$ to $140 \AA$ ). Huxtable et 
al. $[149,150]$ measured the cross-plane thermal conductivity of Si/Ge, Si/SiGe and $\mathrm{SiGe} / \mathrm{SiGe}$ superlattices with periods ranging from 45 to $300 \AA$. The results of some of these measurements are shown in Fig 16.

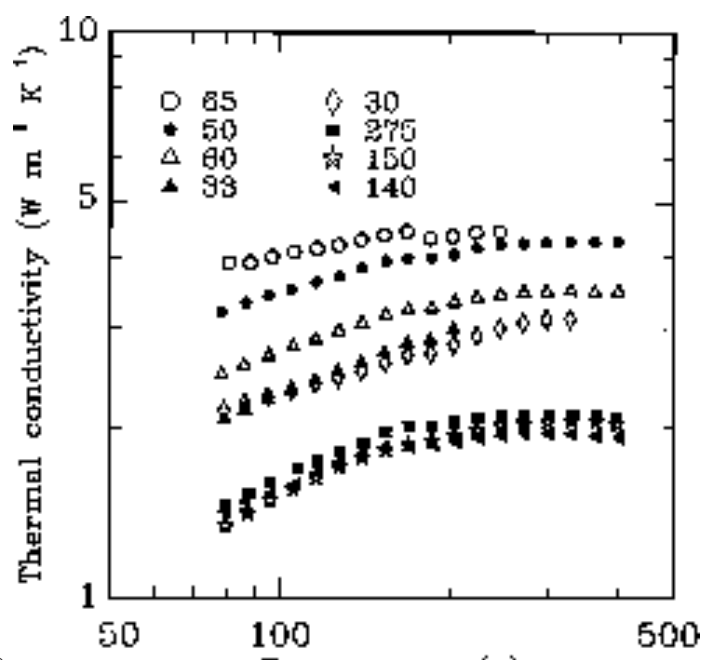

(a)

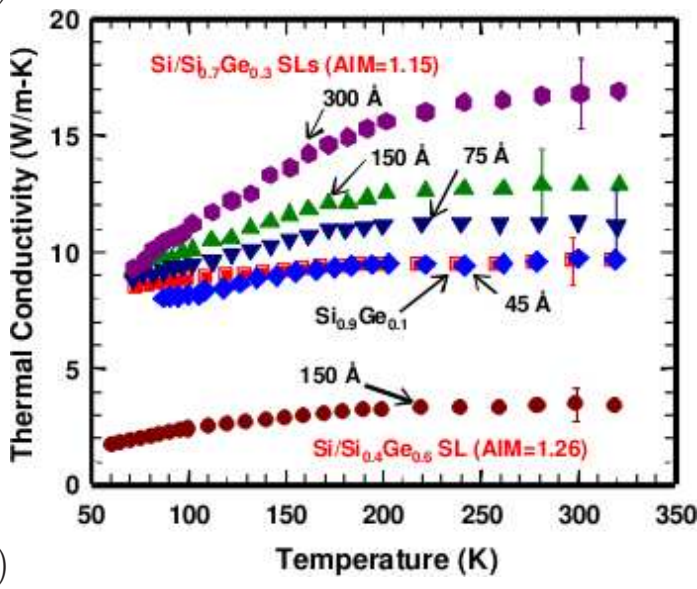

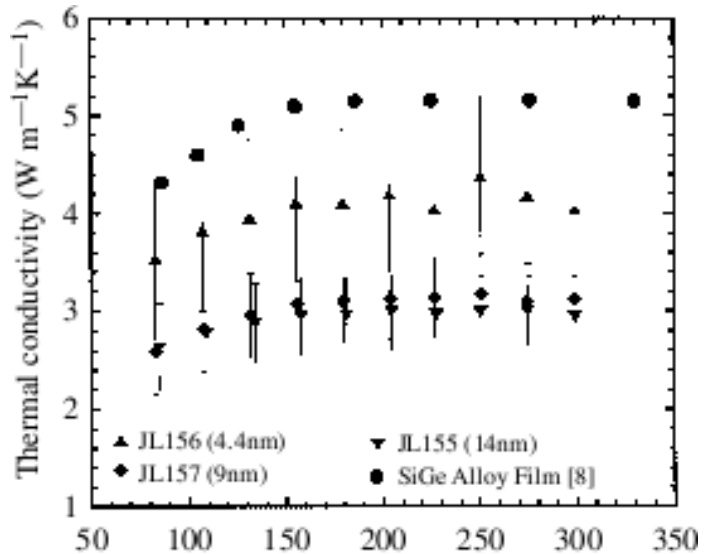

(b)

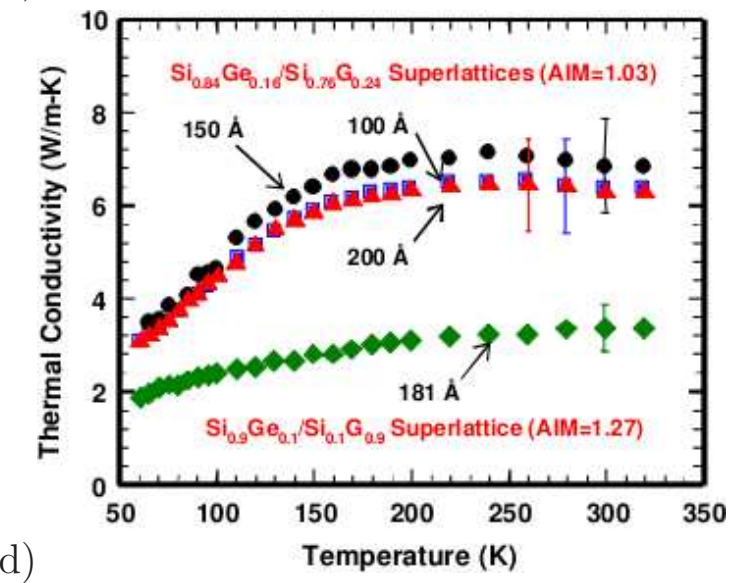

Figure 16. Experimentally measured cross-plane lattice thermal conductivity of $\mathrm{Si} / \mathrm{Ge}$ (panels (a) and (b)), Si/SiGe (panel (c)) and SiGe/SiGe (panel (d)) superlattices. Results are taken from Refs. [147] (panel (a)) [148] (panel (b)) and [150] (panels (c) and (d)).

A few observations can be made and trends established for the conductivity of short-period SLs (periods in the range 40-70 $\AA$ ). (i) There is a strong reduction in the thermal conductivity of a $\mathrm{Si} / \mathrm{Ge}$ superlattice sample relative to the SiGe alloy with comparable fractions of $\mathrm{Si}$ and Ge. (ii) From the plots of results from two groups shown in Fig. 17 it can be said that the conductivity shows a decreasing trend as the period thickness increases. The data from Lee et al. show a dip in the conductivity at around $65 \AA$. Venkatasubramanian and Colpits [151] have suggested that this dip has a physical rather than an experimental origin. However, there is confirmation of this observation from the data points from the sample series used by Borca-Tasciuc et al. [148]. (iii) Lower values of the conductivity of $\mathrm{Si} / \mathrm{SiGe}$ and $\mathrm{SiGe} / \mathrm{SiGe} \mathrm{SLs}$ are obtained for higher 


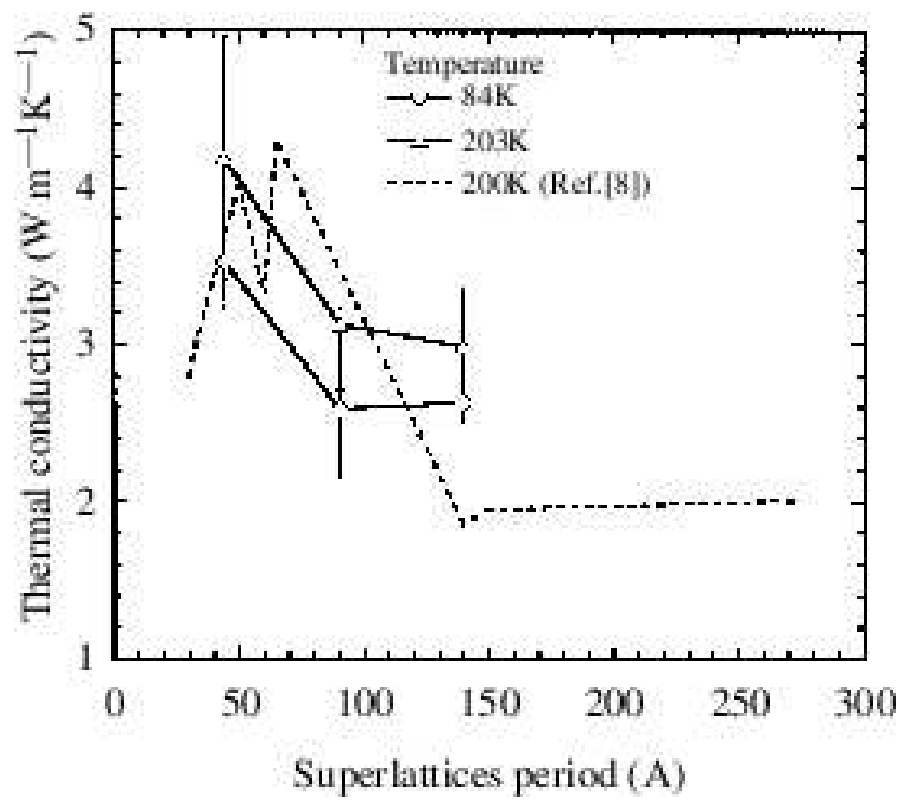

Figure 17. Period thickness variation of experimentally measured cross-plane lattice thermal conductivity of Si/Ge superlattices. The solid and dashed curves represent results from Refs. [148] and [147], respectively. Reproduced from Ref. [148].

Ge concentrations. This is totally consistent with the knowledge that the conductivity of bulk Ge is lower thsn that of bulk Si. (iv) Different growth conditions and techniques for samples may result in different conductivity values. This is clearly seen from a comparison of the top and bottom curves in Fig. 16(d), which shows that for the Si/Ge SL of period $50 \AA$ the conductivity is higher for the sample used by Lee et al. than for the sample used by Huxtable et al. . (v) While the conductivity of Si/Ge SLs does fall below that of the SiGe alloy, the conductivity of Si/SiGe SLs does not seem to fall below the conductivity of the comparable SiGe alloy. (vi) The conductivity of $\mathrm{Si} / \mathrm{SiGe}$ SLs increases with increase in period thickness. Huxtable et al. attribute this to an effective decrease in the number of interface defects per unit length as the period thickness increases. (vii) The conductivity of SiGe/SiGe SLs is found to be independent of the period thickness.

There is no trend or firm explanation of the behaviour observed for the cross-plane conductivity of superlattices. Similarly, there is no report of systematic measurement, or understanding, of the ratio of the parallel-plane and cross-plane conductivities of a superlattice. There is also no systematic observation of the dependence of the conductivty on the physical size (number of periods or total length) of a superlattice. Different growth conditions and techniques are likely to fabricate samples of different quality. One of the consequences of this is that samples fabricated by different techniques may have different interface attributes and thus scatter phonons differently. Some of these issues have recently been the subject of careful theoretical investigations, as discussed next. 
Garg et al. [152] computed the lattice thermal conductivity of an ideal short period $\left(\mathrm{Si}_{0.5} \mathrm{Ge}_{0.5}\right)_{n}\left(\mathrm{Si}_{0.5} \mathrm{Ge}_{0.5}\right)_{n}[001]$ superlattice using the DFPT and the single-mode relaxation time approximation. Each unit cell of such a periodic structure contains a total of $2 n$ atomic layers, with each of $n$ atomic layers of Si alternating with each of $n$ atomic layers of Ge. Although such a structure may be unrealistic to fabricate, the results for such a system do provide some useful insight into the conductivity results of ultra-thin SLs. Their results, presented in Fig. 18, indicates that above $150 \mathrm{~K}$ the conductivity of the ideal (isotopically pure) $\left(\mathrm{Si}_{0.5} \mathrm{Ge}_{0.5}\right)_{1}\left(\mathrm{Si}_{0.5} \mathrm{Ge}_{0.5}\right)_{1}[001] \mathrm{SL}$ is higher than that of both the constituent materials. The conductivity of this SL is higher than that of a virtual "average material", i.e. what can be computed within the virtual crystal approximation for the SL structure. The in-plane conductivity is computed to be larger than the cross-plane conductivity for the layer index $n$ larger than unity.

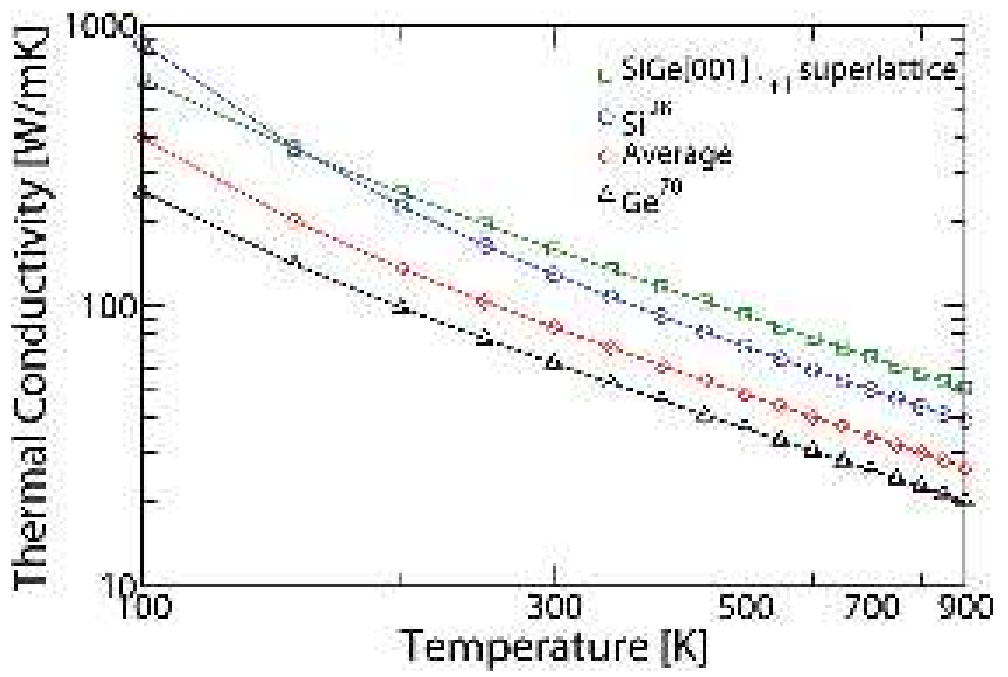

Figure 18. Lattice thermal conductivity of a fictitious $\left(\mathrm{Si}_{0.5} \mathrm{Ge}_{0.5}\right)_{1}\left(\mathrm{Si}_{0.5} \mathrm{Ge}_{0.5}\right)_{1}[001]$ superlattice. Reprinted with permission from [152]. Copyright (2011) Americal Chemical Society.

A detailed and more complete investigation of the lattice thermal conductivity of short-period semiconductor superlattices has been undertaken by Srivastava's group [89, 90, 127, 128]. They computed the conductivity using the expression in Eq. (83) and the single-mode relaxation time expressions in Eqs. (104), (106), (108), (109), (116) and (130). The phonon dispersion curves in Refs. [127, 128] and [89, 90] were obtained by employing the adiabatic bond charge model and the DFPT, respectively. There are essentially three physical properties that can alter the conductivity of a superlattice compared to that of its constituent materials: specific heat at constant volume $C_{v}$, phonon speed $c$ and phonon relaxation time $\tau$. Results in Sec. 4.2 suggest that there is generally a decrease in phonon speed $c$ upon SL formation. However, such a decrease is far smaller than by an order of magnitude. Numerical results of specific heat for bulk Si and Ge, and for the $\mathrm{Si}_{(20-n)} \mathrm{Ge}_{n}[001]$ superlattices (comprised of 20-n monolayers of $\mathrm{Si}$ and $n$ monolayers of Ge per unit cell), are presented in Fig. 19. Regardless of the layer 
index $n$, the $C_{v}(\mathrm{SL})$ generally lies between $C_{v}(\mathrm{Ge})$ and $C_{v}(\mathrm{Si})$. It is noted that $C_{v}(\mathrm{SL})$ is approx imately $14 \%$ lower than the average values for bulk $\mathrm{Si}$ and Ge. This suggests that upon superlattice formation the specific heat does not significantly deviate from the weighted average of the bulk values. It is therefore clear that a systematic and accurate calculation of the phonon relaxation time should be made to investigate the observed reduction in the lattice thermal conductivity upon superlattice formation comprised of two or more constituent bulk materials. The formulas presented in Sec. 4.4 suggest that the phonon relaxation rates due to the IMS, IDS and anharmonicity depend on several factors, including the amplitude ratio $e_{B} / e_{A}$ between the two materials $\mathrm{A}$ and $\mathrm{B}$ in a a $\mathrm{A} / \mathrm{B}$ superlattice, phonon dispersion relations, superlattice period, the interface parameters $F_{I M S}$ and $F_{I D S}$ and the temperature dependent anharmonicity parameter $\gamma(T)$. Of these parameters, the amplitude ratio $e_{B} / e_{A}$ varies most sensitively with the phonon wave vector $q_{z}$ along the SL growth direction: while $e_{B} / e_{A}$ is unity at the zone centre, it rises towards infinity at the zone edge $\left(l_{z} q_{z}=\pi / 2\right)$. Keeping this in mind we can make a few qualitative statements regarding phonon relaxation rates from different scattering sources.

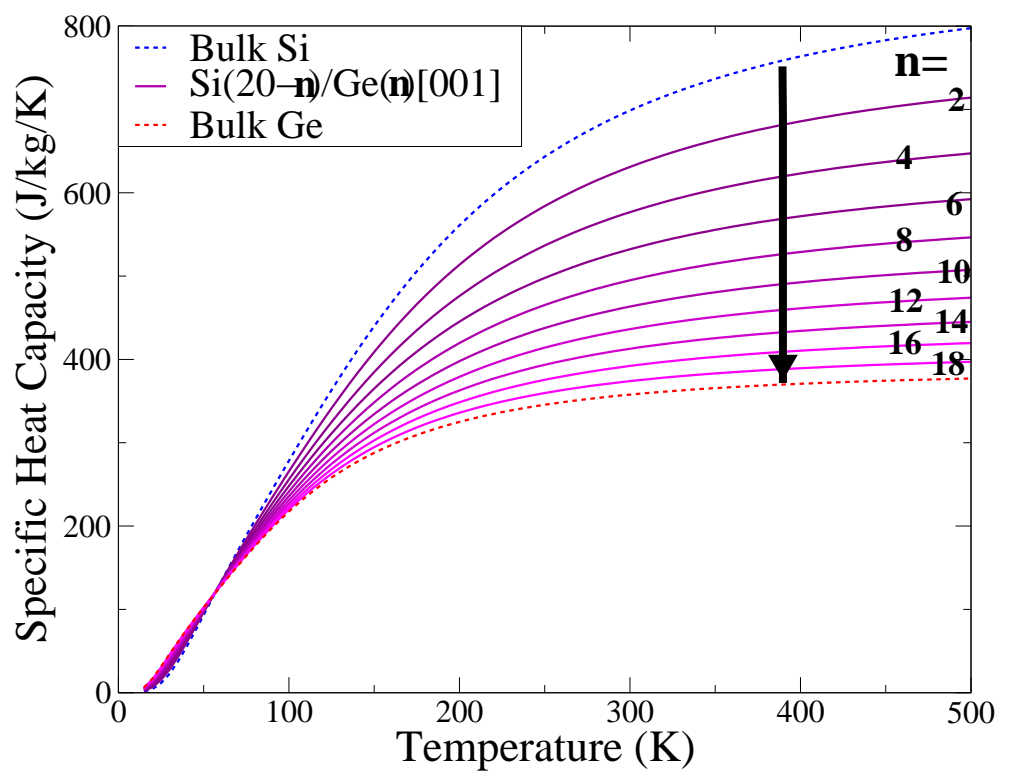

Figure 19. Comparison of the lattice specific heat of $\left.\mathrm{Si}_{(} 20-n\right) \mathrm{Ge}_{n}[001]$ superlattices with bulk Si and Ge. Reproduced from [128].

For phonon modes near the zone edge in the growth direction, the two material regions in each superlattice unit cell will act out of phase and, hence, traveling phonons will be scattered more strongly by the presence of the interfaces. For both IMS and IDS scattering mechanisms, optical modes in superlattices will be scattered at the interface more strongly than acoustic modes. Sharp peaks in the density of states in the optical range, due to strongly confined modes with much reduced velocities, will result in the scattering rate of optical modes being greater than for their acoustic counterparts. Thus we can expect that interfaces will weakly scatter long wavelength modes. The factor 
$1 /\left(n_{1}+n_{2}\right)^{2}$ in Eqs. (137) and (138) implies that the IMS and IDS mechanisms become more powerful as the superlattice period decreases. In other words, phonon interface scatterings are greatest for thin superlattices and decrease as the superlattices become thicker. However, one must also consider that the thinner the two regions, the smaller the region that becomes available for mass smearing and broken bonds.

Phonon anharmonic relaxation rates in superlattice structures are affected by three factors: zone-folding effect in phonon dispersion relations (i.e. changes in frequencies and velocities), mini-gap formations, and the dual mass term $D$ (due to the presence of two materials in a A/B superlattice). The first two of these factors can produce two seemingly opposite effects: reduction in the number of allowed three-phonon interactions involving acoustic and/or optical modes, and reduction in the number of phonon modes taking part in conduction process. The third factor, the dual mass term, is controlled by the amplitude ratio $e_{B} / e_{A}$ and the density ratio $\varrho_{A} / \varrho_{B}$ (or, the mass ratio $M_{A} / M_{B}$ ). As discussed earlier, superlattice structure formation with the presence of more than one material per unit cell also gives rise to the development of 'mini-Umklapp' processes. As $e_{B} / e_{A}$ grows exponentially towards the zone edge, there will be a much greater increase in the relaxation rate for zone-edge modes compared to zone-centre modes. From the points made above, we can expect that the relaxation rate of acoustic phonons will be more affected by the dual mass term $D$ and that the relaxation rate of optical modes will be affected by both the mini-gap formation and the $D$ term.

When attempting a first-principles calculation of the lattice thermal conductivity of a nanocomposite, the first obvious thing to do is to 'relax' the crystal structure. Referring to a SL structure, this involves obtaining the equilibrium lattice constant and relaxed atomic positions at the interface between the two materials inside each unit cell. The effect of interface relaxation on phonon dispersion relation and thermal conductivity of ultra-thin Si/Ge SLs was investigated in Ref. [89]. It was found that interface relaxation results in important changes in the phonon spectrum above $200 \mathrm{~cm}^{-1}$ in ultrathin $\mathrm{Si}_{n} \mathrm{Ge}_{n}$ [001] SLs, with $n$ representing the number of atomic bilayers. This in turn results in alterations to mini-gap locations and widths. The product of the specific heat and phonon velocity squared is more affected by the geometry relaxation along the SL growth direction than in a SL plane direction. The zone-average value of the phonon anharmonic relaxation time in $\mathrm{Si}_{1} \mathrm{Ge}_{1} \mathrm{SL}$ is increased by around $7 \%$. However, as shown in Fig. 20, the overall effect of atomic relaxation is to reduces the conductivity tensor components by no more than $5 \%$. We note that in this figure $x, y$ and $z$ axes are taken along [110], [110] and [001], respectively. The calculations were made with both alloy and isotope mass scatterings included, and the anharmonic interaction included with the choice of Grüneisen's constant $\gamma=0.5$.

Detailed numerical calculations of the lattice thermal conductivity of $\operatorname{Si}_{n} \mathrm{Ge}_{n}[001]$ superlattices, with $n=1-8$ indicating atomic bilayers, have been presented in Ref. [90]. In this article we have also expressed these structures by $\operatorname{SL}(n, n)$. For such ultrathin SLs there is no evidence of the development of interface misfit dislocation and thus no IDS was included. A revised scheme for mass smudging across interfaces 


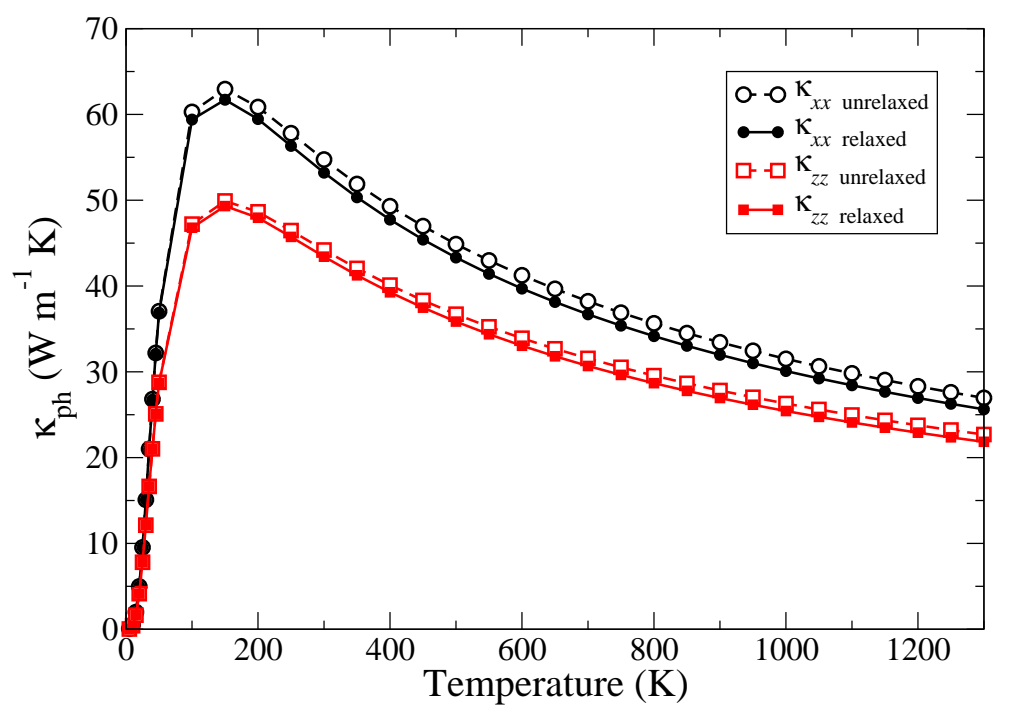

Figure 20. Effect of atomic relaxation on the lattice thermal conductivity of $\mathrm{Si}_{1} \mathrm{Ge}_{1}$ superlattice. Reproduced from [89].

was adopted for deriving the IMS relaxation rate. Experimental measurements have indicated that the anharmonicity parameter Grüneisen's constant $\gamma$ is temperature dependent for bulk Si and Ge, usually increasing with temperature [153, 154]. Assuming a similar behaviour for $\mathrm{Si} / \mathrm{Ge} \mathrm{SLs}$, test results for $\mathrm{SL}(1,1)$ indicated that the following temperature-dependent form of mode-average $\gamma$ is a reasonable choice:

$$
\gamma=\left\{\begin{array}{ll}
\gamma_{0} & T<T_{c} \\
\gamma_{0}\left(1+\frac{\left(T-T_{c}\right)}{T_{c}}\right)^{n_{\gamma}} & T \geq T_{c}
\end{array},\right.
$$

with $\gamma_{0}=0.45, n_{\gamma}=0.56$ and $T_{c}=150 \mathrm{~K}$. The choice of these parameters produces values of $\gamma$ that lie intermediate to values obtained experimentally for bulk Si and Ge, and close to the value for SiGe alloys in the temperature range 150-300 K [154].

Figure 21 shows the results for the conductivity tensor components for SL $(1,1)$ and $\mathrm{SL}(8,8)$. Also shown in panel (a) are the experimental data for bulk Si and Ge from Ref. [155] and in panel (b) the experimental data from Ref. [148] for the $\operatorname{Si}(22 \AA) \mathrm{Ge}(22$ $\AA$ ) superlattice (which is close to $\mathrm{SL}(8,8)$ ) with a sample size equivalent to 100 periods. The figure caption details the mass-defect scattering parameter, the probability factor for interface mass-mixing and the boundary length used in the calculations.

The conductivity tensor $\kappa_{i j}$ for $\mathrm{SL}(1,1)$ is isotropic, since the system can be viewed as quite similar to a bulk alloy. Based on a reasonable defence of the parameters employed in the calculations, it has been predicted that the low-temperature conductivity components of $\mathrm{SL}(1,1)$ would be significantly lower (by an order of magnitude) than the average of results for equivalent bulk $\mathrm{Si}$ and $\mathrm{Ge}$ systems. At temperatures above $100 \mathrm{~K}$, the SL(1,1) conductivity components would lie intermediate to the results for bulk $\mathrm{Si}$ and Ge. These predictions are at variance with those presented by Garg et al. [152] in Fig. 18 for the monolayer Si/Ge superlattice. 
(a)
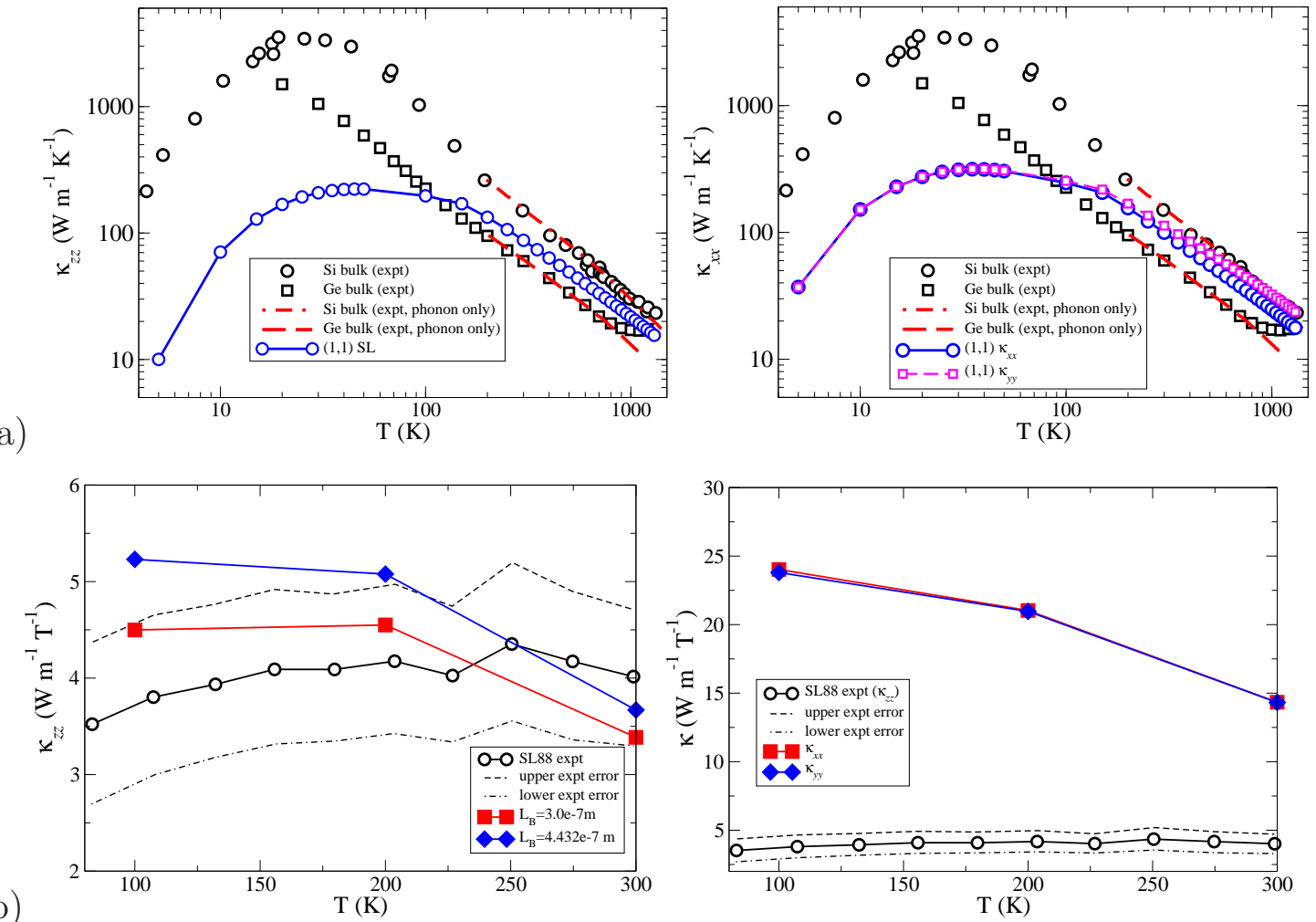

Figure 21. Lattice thermal conductivity tensor components for the ultrathin $\mathrm{Si}_{1} \mathrm{Ge}_{1}[001]$ and $\mathrm{Si} 8 \mathrm{Gen}[001]$ superlattices, where $n$ indicates the number of atomic bilayers. The parameter $L_{B}$ represents the effective boundary scattering length. The point mass-defect scattering rate is scaled by a factor of 4.5 and a probability of 0.05 is used for modelling mass mixing at an interface layer. Reproduced from [90].

A clear difference between the in-plane conductivity components $\left(\kappa_{x x}\right.$ and $\left.\kappa_{y y}\right)$ and cross-plane component $\kappa_{z z}$ can be noted from Fig. 21(b) for $\operatorname{SL}(8,8)$. The inplane components are predicted to be approximately 4 times larger than the cross-plane component. The relative reduction in the $\kappa_{z z}$ component is obviously due to two main factors: reduced phonon velocity and the IMS factor. The experimental results of Ref. [148] have been compared with two calculations for $\operatorname{SL}(8,8)$. In both calculations the same point mass-defect scale factor and interface mass-mixing probability factor were used, but two different boundary lengths were considered: $L_{B}=4.43 \times 10^{-7} \mathrm{~m}(100$ period value, the actual sample size) and $L_{B}=3 \times 10^{-7} \mathrm{~m}$ (around $68 \%$ of the sample size). There is good qualitative agreement between theory and experiment. However, the agreement is improved for the smaller boundary length, which can be considered to indicate some measure of surface roughness.

Variation of $\kappa_{z z}$ with the period size (i.e. with the atomic bilayer index $n$ ) is found to be rather complicated. While in general $\kappa_{z z}$ decreases with $n$, the precise manner in which this happens depend on the details of interface mass mixing (e.g. probability of a layer being mixed and the number of layers being mixed). Interface quality is largely dependent on growth conditions and the techniques employed. 

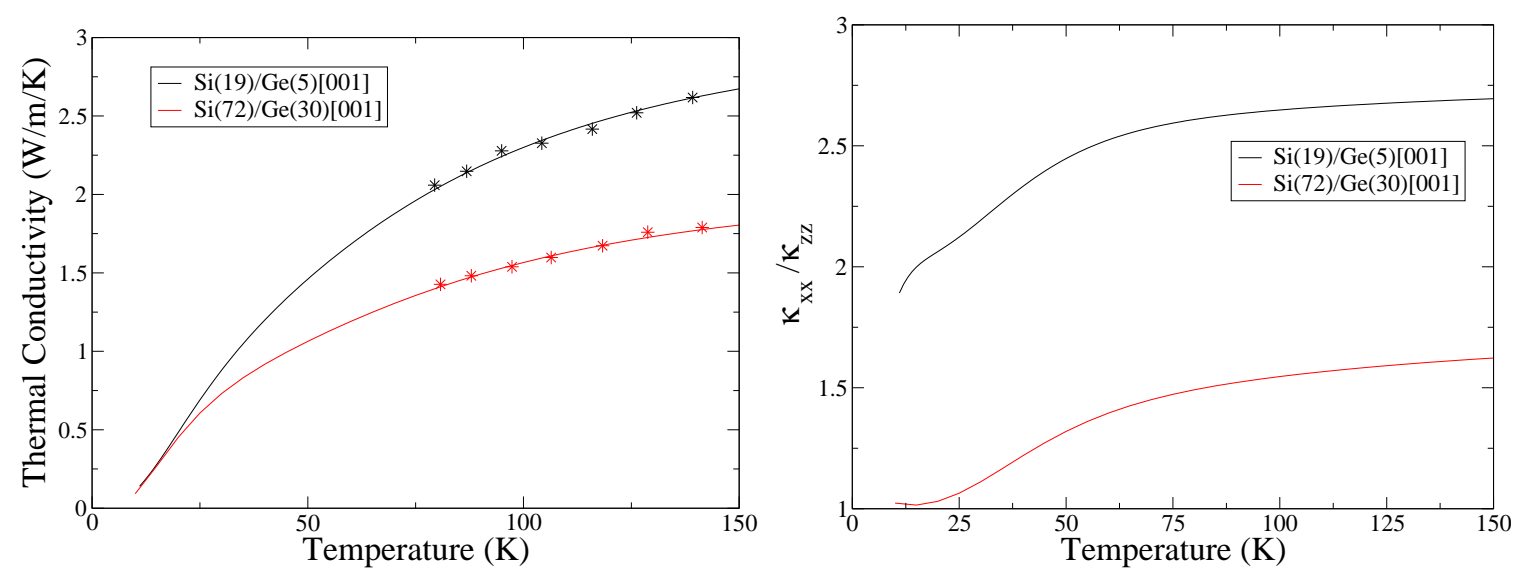

Figure 22. Lattice thermal conductivity for $\mathrm{Si}(19) / \mathrm{Ge}(5)[001]$ and $\mathrm{Si}(72) / \mathrm{Ge}(30)[001]$ superlattices, where the numbers of atomic monolayers are indicated. Reproduced from [128].

Figure 22 shows the low-temperature conductivity results for for [001] superlattices containing $19 / 5$ and $72 / 30$ Si/Ge monolayers: viz. with SL period sizes $33 \AA$ and 140 $\AA$, respectively. Lee et al. [147] fabricated such SLs and measured their conductivity. For explaining these low-temperature results, the anharmonic phonon interaction was ignored (i.e. $\gamma=0$ was used). Boundary scattering lengths were taken as the sample sizes reported by Lee et al. . The strength of isotopic mass defect scattering was calculated a priori from the relative isotopic abundances. For a successful reproduction of the experimental results for the thinner SL only the interface IMS mechanism was invoked, while for the thicker SL both IMS and IDS mechanisms were considered. From the calculations it was found that beyond $100 \mathrm{~K}$ the conductivity of these SLs is largely controlled by the IMS and IDS mechanisms. The calculations suggest that at very low temperatures, where boundary scattering is governed by the sample size, the conductivities of the two SLs have similar values. Above approximately $25 \mathrm{~K}$, the results are controlled by interface scattering mechanism(s). In accordance with the general trend of decrease of conductivity with increase in SL period size, it is found that both cross-plane and in-plane conductivity components for the thicker SL are smaller than those for the thinner SL. The numerical results show clear anisotropic behaviour of the conductivity. The ratio $\kappa_{x x} / \kappa_{z z}$ tends to unity at very low temperatures (in the boundary scattering regime) for the thicker SL, whereas it remains much greater than unity for the thinner SL. These results can be explained. At low temperatures, only low-lying phonon modes are populated. In the thicker SL large amounts of zone-folding take place and at low temperatures phonon modes both at the zone-centre and the zone-edge are populated. Conversely, for the thinner SL there is less zone-folding and the zone-edge modes are not populated until higher temperatures. In accordance with Eq. (133), zone-edge modes are scattered more strongly than zone-centre modes, making both IMS and IDS highly anisotropic scattering mechanisms at low temperatures for the thinner SL, but less so for the thicker SL. 
(ii) Thin Si nanowires:

Li et al. [156] have used the vapour-liquid-solid method to synthesize Si nanowires of different diameters (22, 37, 56, and $115 \mathrm{~nm})$. Symbols in Fig. 23 show the measured thermal conductivities of their samples. These authors were able to fit the measured low-temperature data (between 20 and $60 \mathrm{~K}$ ) for the nanowires with 115 and $56 \mathrm{~nm}$ diameters with the Debye $T^{3}$ law. However, for the smaller diameter nanowires (37 and $22 \mathrm{~nm}$ ) the temperature power exponent becomes smaller as the diameter decreases, and for the $22 \mathrm{~nm}$ nanowire a significant deviation from the $T^{3}$ law was established.

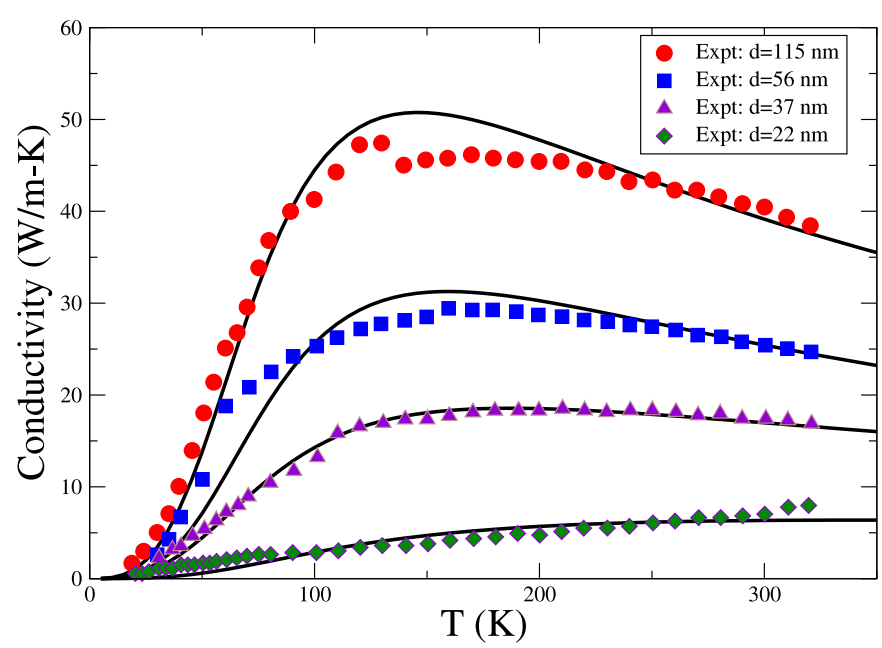

Figure 23. Lattice thermal conductivity of Si nanowires. The experimental data is read from [156] and the figure is reproduced with permission from [160].

Several theoretical attempts have been made to explain the experimental results obtained by Li et al. , including the semi-classical Boltzmann-type approach [157], a semi-empirical molecular dynamics approach [158], and the quantum Landauer formalism [159]. All these models are capable of explaining the measured thermal conductivity results for the nanowires of all diameters, except $22 \mathrm{~nm}$. It has been argued [159] that in order to explain the conductivity of the nanowire of diameter $22 \mathrm{~nm}$ it is important to employ a theoretical model that combines incoherent surface scattering for short-wavelength phonons with nearly ballistic long-wavelength phonons. However, a later theoretical attempt [160] employing the Callaway-Debye-Srivastava relaxation-time model (i.e. using Eqs. (102), (104), (105), (107) and (125)) was found to successfully explain the results for all diameter sizes, including $22 \mathrm{~nm}$. The theoretical results from Ref. [160] are shown by the solid curves in in Fig. 23.

For reproducing the experimental results for the nanowires of diameters $115 \mathrm{~nm}$ and $56 \mathrm{~nm}$ numerical calculations had to use a several times stronger mass-defect scattering 
parameter than what is needed for bulk Si. For the thinner nanowire of diameter $37 \mathrm{~nm}$, the mass-defect parameter had to be increased five-fold compared to that for the thicker nanowires. For the thinnest nanowire (diameter $22 \mathrm{~nm}$ ) it became necessary to consider a very strong diffuse surface (boundary) scattering, and a mass-defect scattering parameter twice that for the $37 \mathrm{~nm}$ wire. The choice of a significantly smaller effective boundary length for the $22 \mathrm{~nm}$ wire clearly indicates that the surface of this wire is quite rough. For nanowires of all thickness, a stronger anharmonicity factor (Grünisen's constant $\gamma$ between 1.4 and 1.7) was used than is needed for bulk Si (usually $\gamma=0.8$ is adequate). Following the discussion in Sec. 4.3.2, we re-iterate that the level of successful agreement between theory and experiment for the conductivity achieved for the $22 \mathrm{~nm}$ nanowire is not expected to continue to much thinner nanowires.

\section{(iii) Nanodots dispersed in a homogeneous host matrix:}

Behrang et al. [135] have presented results of their theoretical investigations for the lattice thermal conductivity of Ge (host) in which spherical Si particles (NDs) are dispersed. For this they used the single mode relaxation time expression within Debye's isotropic continuum scheme (the first term in Eq. (102) and fitted experimental conductivity results for bulk $\mathrm{Si}$ and Ge by fitting the adjustable parameters $B$ and $\theta$ in an expression for the anharmonic mean free path of the form $\Lambda^{-1}=B T \omega^{2} \exp (-\theta / T)$. With bulk results fitted, they computed the effective thermal conductivity of the composite material by using the formula

$$
\kappa_{\text {eff }}=\kappa_{\text {host }} \frac{2 \kappa_{\text {host }}+(1+2 \alpha) \kappa_{N D}+2 \phi\left[(1-\alpha) \kappa_{\mathrm{ND}}-\kappa_{\text {host }}\right]}{2 \kappa_{\text {host }}+(1+2 \alpha) \kappa_{N D}-\phi\left[(1-\alpha) \kappa_{\mathrm{ND}}-\kappa_{\text {host }}\right]},
$$

where $\phi$ is the volume fraction of the suspended particles, and $\kappa_{\text {host }}$ and $\kappa_{\mathrm{ND}}$ are the conductivites of the host $(\mathrm{Ge})$ and nanodots (Si), respectively. The effective phonon mean free paths in the host and NDs, $\Lambda_{\text {effective host }}$ and $\Lambda_{\text {effective ND, are computed using }}$ the scattering formulae presented in Sec. 4.4.2.

The results of their calculations for different Si nanodot sizes and volume fractions in Ge host are presented in Fig. 24. A few observations can be made. The conductivity of the nanocomposite is lower than that of bulk Ge at all temperatures for all Si nanodot sizes and volume fractions. At a given temperature, the conductivity decreases with a decrease of the nanodot size. The conductivity decreases with an increase in the volume fraction of the nanodots. For the volume fraction $\phi=0.2$ of the smallest nanodot considered $(10 \mathrm{~nm})$, the conductivity becomes almost temperature independent. This is due to the dominance of the interface scattering over the anharmonic scattering over the whole temperature range of the calculations (200-1000 K). The significantly reduced values of the conductivity at higher volume fractions and smaller particle sizes is expected to arise due to the relative increase in the interface scattering area per unit volume [161] implying strong confinement of phonon transport at the particle-host interface.

Mingo et al. [162] employed a "nanoparticle-in-alloy" material approach to investigate the $Z T$ of nanoparticles embedded in an alloy matrix. It was found that the 

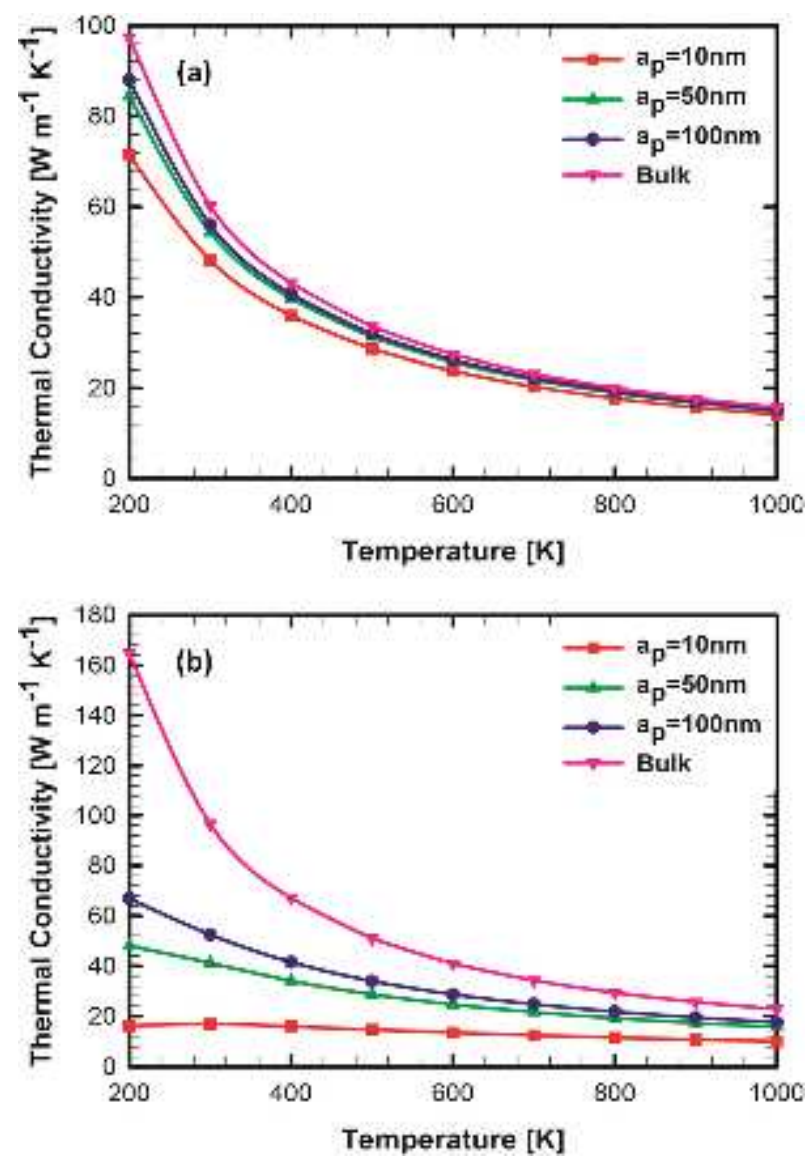

Figure 24. Lattice thermal conductivity of Si nanodots dispersed in a Ge host. Results of theoretical calculations are presented for different nanodot sizes $\left(a_{p}\right)$ and volume fractions $(\phi)$. Panel $(\mathrm{a}): \phi=0.01$, panel $(\mathrm{b}): \phi=0.2$. For comparison, results for bulk Ge are also presented. Reproduced with permission from [135]. Copyright (2013), AIP Publishing LLC.

effect of embedding silicide and germanide fillers led to a potential 5 -fold increase in $Z T$ of SiGe alloys at room temperature and 2.5 times increase (making $Z T \sim 1.7$ ) at 900 $K$. This work predicts the existence of an optimal nanoparticle size that minimizes the nanocomposite's lattice thermal conductivity.

(iv) Finite-length nanowires dispersed in a homogeneous host matrix:

More recently, Behrand et al. [163] have adopted the method decribed in the preceeding subsection for deriving a formula for the heat conductivity of a system containing a dispersion of generally oriented nanowires of a finite length in a homogeneous matrix. For such a system, the conductivity becomes a function of four parameters: the specularity parameter $p$, the length $L_{W}$ of the nanowires, the radius $a_{p}$ od the nanowires, and the angle $\theta$ between the nanowire orientation and the direction of the heat flux. Their numerical calculations for Si nanowires in a Ge host show that for all volume fractions the longitudinal conductivity $\kappa_{\|}$(i.e. along the nanowire growth direction) 
increases with the nanowire length $L_{W}$ and approaches a plateau al larger lengths. Also, $\kappa_{\|}$decreases with increasing the nanowire volume fraction, due to a relative increase in phonon-interface scattering. The conductivity generally decreases as the orientational angle $\theta$ increases from zero (for $\left.\kappa_{\|}\right)$to $\pi / 2$ (for $\kappa_{\perp}$ ). This clearly indicates that the conductivity becomes strongly anisotropic. The anisotropic factor $\left(\kappa_{\|}-\kappa_{\perp}\right) / \kappa_{\|}$could be changed in a controlled manner by appropriately choosing the four parameters mentioned earlier. Their numerical calculations suggest that the anisotropic factor of $42 \%$ could be achieved for $\phi=0.1, a_{p}=10 \mathrm{~nm}, L_{W}=300 \mathrm{~nm}$, and $p=0$.

(v) General remark on 'tuning' of lattice thermal conductivity via nanostructuring:

It has been demonstrated both from experimental measurements and theoretical calculations that nanostructuring (e.g. in the form of superlattices, nanowires, and nanodots dispersed in the host matrix) is capable of producing a significant reduction in lattice thermal conductivity compared to the corresponding bulk value(s). Different amounts of reduction can be achieved from different types of nanostructuring.

The results presented in Figs. 16 and 17 indicate that the room-temperature conductivity values in the range $2-15 \mathrm{Wm}^{-1} \mathrm{~K}^{-1}$ can be achieved by controlled formation of $\mathrm{Si} / \mathrm{Ge}, \mathrm{Si} / \mathrm{SiGe}$ and $\mathrm{SiGe} / \mathrm{SiGe}$ superlattices with their period size in the range 3-30 $\mathrm{nm}$. The results presented in Fig. 23 indicate that conductivity values in the range 5-40 $\mathrm{Wm}^{-1} \mathrm{~K}^{-1}$ can be achieved for Si nanowires in size range $22-115 \mathrm{~nm}$. Finally, the results in Fig. 24 predict that the conductivity of the nanocomposite made of a small fraction of Si nanodots in the size range 10-100 nm dispersed in the Ge host can expected to be in the range $10-50 \mathrm{Wm}^{-1} \mathrm{~K}^{-1}$. It is clear therefore that a great deal of 'tunability' of the conductivity is possible by fabrication of different types and sizes of nanocomposites.

However, as remarked in Sec. 4.4.2(iv), there are several obscure variables that might individually and/or collectively control phonon scatterings in nanostructures and nanocomposites. More experimental and theoretical research is required to determine the ranges and relative strengths of such parameters so as to determine the degree of 'tunability' of the conductivity of such materials.

\section{Examples of phonon tuning for the enhancement of TE figure of merit}

Having presented some examples of the extent to which phonon conductivity can be controlled (reduced) via nanostructuring, we will now discuss the role of phonon tuning in the enhancement of TE figure of merit. It has long been the aim of therists to do so, but the lack of theoretical schemes and computational resources to deal with the phonon conductivity of bulk and nanostructured materials using the same theoretical technique and incorporating relevant phonon interaction processes has hindered the progress in terms of presenting numerically accurate answers for ZT. As mentioned in Section 5.3, different levels of theoretical and computational efforts for $\kappa_{\mathrm{ph}}$ are required for different sizes (widths) of nanocomposite materials. Also, as mentioned in the preceeding section, several theoretical/computational groups [164] are actively engaged in first-principles 
calculations of the phonon conductivity. However, recipes for dealing with anharmonic phonon interactions and phonon-interface scatterings have not yet been adequately developed and tested. To the best of this author's knowledge, ours is the first group to deal with these two types of phonon interactions for nanocomposites. These theories, described in Sects. 4.4.2 (i) and (ii), by no means provide the best recipes, but their effects have been systematically included in the $\kappa_{\mathrm{ph}}$ results presented in the preceeding section. In particular, we remind the reader of the 'obscure' parameters that may control $\kappa_{\mathrm{ph}}$ of nanocomposites.

In this section we aim to discuss progress made, using systematic theoretical and computational approaches, in examining the role of phonon tuning in the enhancement of $Z T$. As an enhancement of $Z T$ can be governed both by the electronic tuning (by increasing the power factor) as well as the phonon tuning, we will also comment on the extent to which nanostructuring has been found to help the former.

(i) SiGe alloys:

Thomas and Srivastava [123] made calculations of the TE coefficients for an n-type $\mathrm{Si}_{0.754} \mathrm{Ge}_{0.246}$ alloy doped with $\mathrm{P}$ donor impurities of concentration $7.3 \times 10^{25} \mathrm{~m}^{-3}$. The results obtained for the Fermi level and Seebeck coefficient from the theoretical analysis are shown in Fig. 25. Throughout the temperature range 200-1200 K the Fermi level lies in the upper part of the electronic band gap of the alloy, clearly indicating extrinsic-type behaviour. Good agreement with experimental data for $S$ from Meddins and Parrott [141] provides confirmation that there is no obvious transition to a region of intrinsictype electrical behaviour.

(a)

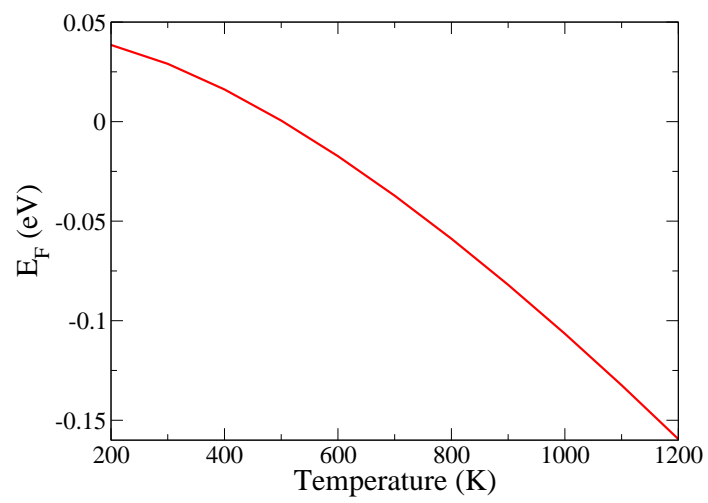

(b)

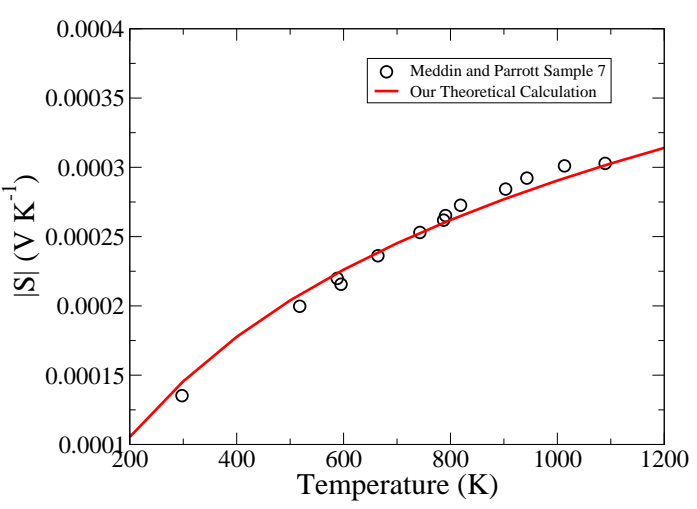

Figure 25. Temperature variation of (a) Fermi energy (below the conduction band minimum) and (b) Seebeck coeffcient in an n-type $\mathrm{Si}_{0.754} \mathrm{Ge}_{0.246}$ alloy doped with $\mathrm{P}$ donor impurities of concentration $7.3 \times 10^{25} \mathrm{~m}^{-3}$. Solid curves are theoretical results reported in Ref. [123]. The symbols represent experimental measurements from Ref. [141]. Reproduced from [123].

The temperature variation of the electrical conductivity $\sigma$ is presented in Fig. 26(a). It was found that in order to properly account for the temperature dependence of $\sigma$ agreement between theory and experiment required an ad hoc multiplication of the 
theoretical results by a term $0.0013 T^{0.4}$. The necessity for such a fix indicates that a theory beyond the effective mass approximation is required for explaining electrical conduction in doped semiconductors.

(a)

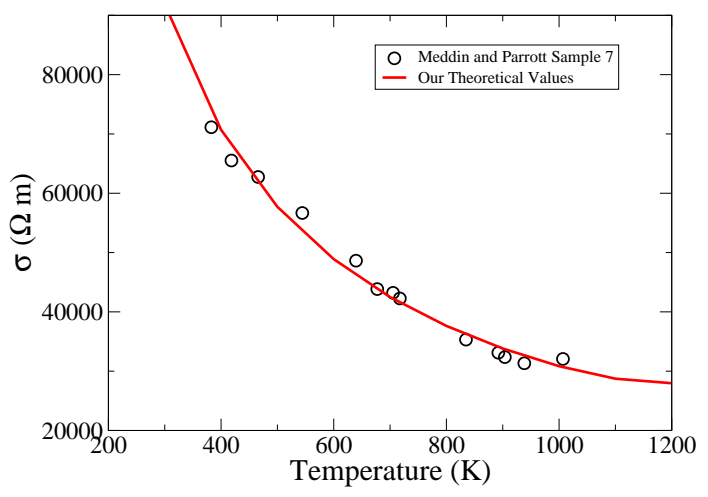

(b)

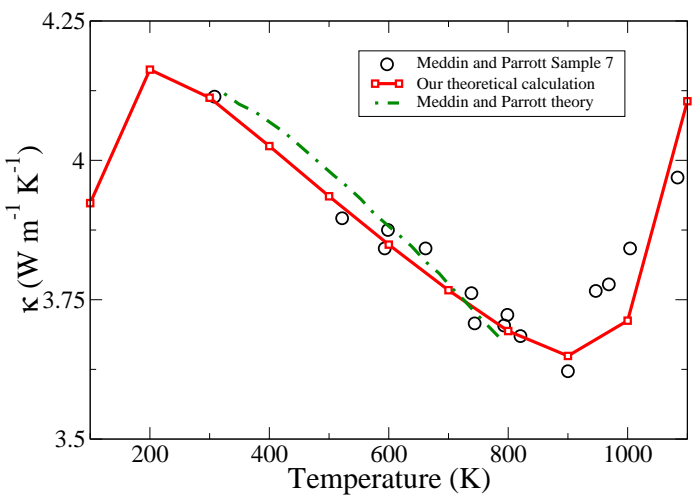

Figure 26. Temperature dependence of (a) electrical conductivity and (b) total lattice thermal conductivity $\left(\kappa=\kappa_{\mathrm{ph}}+\kappa_{\mathrm{el}}\right)$ of an n-type $\mathrm{Si}_{0.754} \mathrm{Ge}_{0.246}$ alloy doped with $\mathrm{P}$ donor impurities of concentration $7.3 \times 10^{25} \mathrm{~m}^{-3}$. Solid and dashed curves are theoretical results reported in Refs. [123] and [141], respectively. The symbols represent experimental measurements from Ref. [141]. Reproduced from [123].

Measured and theoretical results for the total thermal conductivity $\kappa=\kappa_{\text {carriers }}+\kappa_{\text {ph }}$ are shown in Fig. 26(b). The solid curve represents results of calculations of the total thermal conductivity by Thomas and Srivastava [123] using Eqs. (40) and (48) for $\kappa_{\text {carriers }}$, and the first term in Eq. (102) together with Eqs. (104), (106), (108), (109), (116) and (121) for $\kappa_{\mathrm{ph}}$. The dashed curve shows results of an earlier theoretical investigation [141], based on simplified treatments of the transport coefficients. Symbols represent experimental measurements reported in Ref. [141]. There is good agreement between theory and experiment. The magnitude and decrease of the conductivity with increase in temperature in the range $200-600 \mathrm{~K}$ is entirely contributed by phonons. The bipolar contribution $\kappa_{b p}$ begins to become appreciable, rising exponentially with increasing temperature. This makes the total conductivity above $600 \mathrm{~K}$ to exhibit a temperature dependence weaker than that expected from anharmonic interactions and eventually a minimum at around $900 \mathrm{~K}$.

The temperature dependence of the figure of merit $Z T$ is plotted in Fig. 27(a). It is interesting to note that the theoretical calculation by Meddins and Parrott [141] (who employed a simpler and phenomenological theory for $\kappa_{\mathrm{ph}}$ but did not elaborate on their method of calculation of $S, \sigma, \kappa_{r m e l}$ and $\kappa_{\mathrm{bp}}$ ) produced results for $Z T$ vs $T$ which is in qualitative agreement with the results presented by Thomas and Srivastava [123]. In quantitatve terms, there is a discrepancy of around $16 \%$ at $1000 \mathrm{~K}$, and at lower temperatures the results from Thomas and Srivastava are slightly larger than the results from Meddins and Parrott. It is interesting to note from Fig. 27(a) that values of $Z T>0.5$ can be expected throughout the high-temperature range of around 750-1200 $\mathrm{K}$, with a maximum of approximately 0.68 at around $1000 \mathrm{~K}$. 

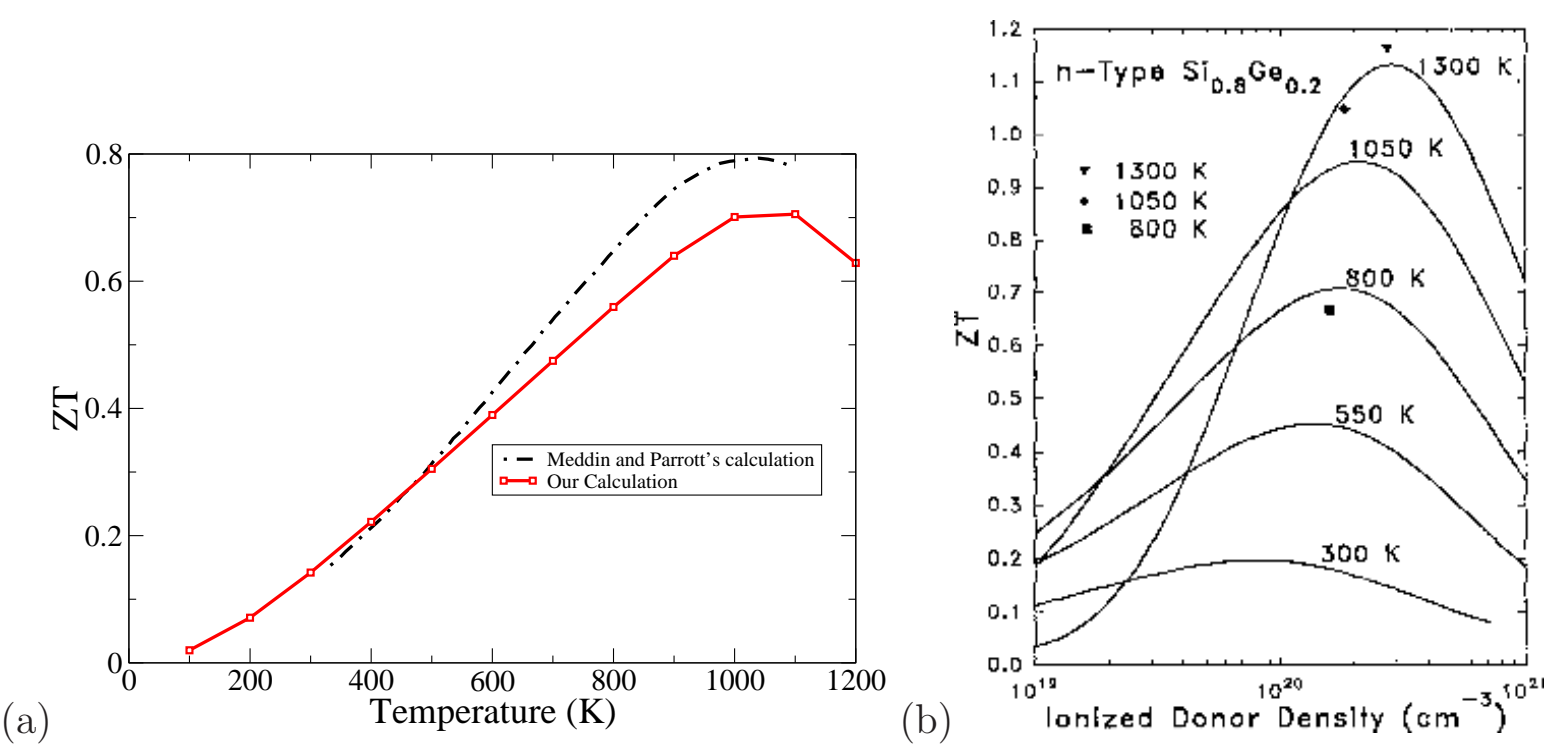

Figure 27. (a) Temperature dependence of the thermoelectric figure of merit for an n-type $\mathrm{Si}_{0.8} \mathrm{Ge}_{0.2}$ alloy doped with $\mathrm{P}$ donor impurities of concentration $7.3 \times 10^{25} \mathrm{~m}^{-3}$. Results of an earlier calculation, from Ref. [141], are also shown. Reproduced from [123]. (b) Figure of merit on n-type SiGe alloys as a function of ionized donor density. Reproduced with permission from [140]. Copyright (1991), AIP Publishing LLC.

Employing similar calculational schemes for $S$ and $\sigma$ (but by treating $E_{F}(T)$ as an adjustable parameter) and the simpler isotropic continuum scheme for phonon conductivity, Vinning [140] obtained similar values of ZT for n-type SiGe alloys. His results, presented in Fig. 27(b), suggest that the optimum donor concentration for achieving highest $Z T$ lies in the range $1-4 \times 10^{26} \mathrm{~m}^{-3}$.

Minnich et al. [165] made a thorough investigation of thermoelectric properties of n-type and p-type SiGe alloys. The electronic transport coefficients were computed by essentially employing the nearly free electron theory discussed in Sect. 3. The phonon conductivity was computed using the Callaway expression in Eq. (102). The threephonon interaction rate was, however, computed by adopting an old and ad hoc scheme [166]. A simple expression for the Umklapp scattering rate of the form $\tau_{U}^{-1} \propto \omega^{2} T$ was considered, the relaxation rate of Normal processes was considered to have a fixed ratio with the Umklapp rate, and the strengths of these scattering rates were fixed in an $a d$ hoc manner. It should be noted that the form of the anharmonic scattering rate used in this work is only valid at high temperatures. A further fitting parameter related to higher-order phonon scattering was also incorporated. The computational details of the phonon conductivity calculations, and the overall conclusions drawn, in this work are quite similar to those in the work by Meddins and Parrott [31] for n-type SiGe alloys. The results obtained in this work are also very similar to those obtained in the work of Vinning [140]. An important obervation is made that with for doping concentration of both n-type and p-type $\mathrm{Si}_{0.7} \mathrm{Ge}_{0.3}$ alloy the bipolar thermal conductivity $\kappa_{\text {bp }}$ is dominant at high temperatures, and is accompanied by a large decrease in Seebeck coefficient. 
(ii) BiTeSe and BiSbTe alloys:

Yelgel and Srivastava [167] computed the $\kappa$ of n-type BiTeSe and p-type BiSbTe alloys by employing the constant relaxation time approach (CRTA) for $S, \sigma$ and $\kappa_{\text {carriers }}$, and Debye's isotropic continnum model within the single-mode relaxation time approach for $\kappa_{\mathrm{ph}}$.

The temperature dependence of the Fermi energy and the Seebeck coefficient in an n-type $\mathrm{Bi}_{2}\left(\mathrm{Te}_{0.85} \mathrm{Se}_{0.15}\right)_{3}$ single crystal, containing $0.1 \mathrm{wt} \% \mathrm{CuBr}$ and $0.2 \mathrm{wt} \% \mathrm{SbI}_{3}$ dopants are shown in Fig. 29. Results of the bipolar contribution and total thermal conductivity are shown in Fig. 30. Their theoretical modelling successfully reproduces the experimental data presented by Hyun et al. [168]. The monopolar electronic contribution $\kappa_{\mathrm{el}}$ decreases with increasing temperature, but the bipolar contribution $\kappa_{\mathrm{bp}}$ starts to become appreciable above $300 \mathrm{~K}$ and increases exponentially. As a result, the total conductivity $\kappa_{\text {tot }}$ shows a minimum around $400 \mathrm{~K}$. The phonon conductivity of the sample with $0.2 \mathrm{wt} \% \mathrm{SbI}_{3}$ dopants is lower than that of the sample with $0.1 \mathrm{wt} \% \mathrm{CuBr}$ dopants. This is the main reason for the minimum in $\kappa_{\text {tot }}$ for the sample with $0.2 \mathrm{wt} \%$ $\mathrm{SbI}_{3}$ dopants to shift to a slightly lower temperature than that for the sample with 0.1 wt\% $\mathrm{CuBr}$ dopants.

(a)

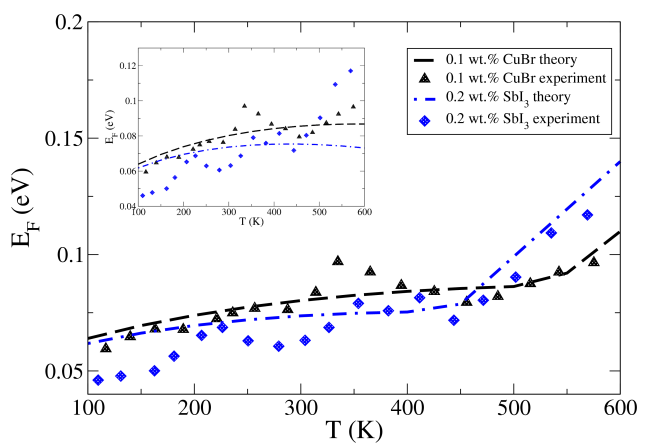

Figure 28. Temperature dependence of the Fermi energy (below the conduction band minimum) and the Seebeck coefficient in an n-type $\mathrm{Bi}_{2}\left(\mathrm{Te}_{0.85} \mathrm{Se}_{0.15}\right)_{3}$ single crystal, containing $0.1 \mathrm{wt} \% \mathrm{CuBr}$ and $0.2 \mathrm{wt} \% \mathrm{SbI}_{3}$ dopants. Reproduced from [167].

The temperature dependence of the Fermi energy, Seebeck coefficient, electrical resistivity and total thermal conductivity in p-type $\left(\mathrm{Bi}_{2} \mathrm{Te}_{3}\right)_{x}\left(\mathrm{Sb}_{2} \mathrm{Te}_{3}\right)_{1-x}$ single crystals doped with 3 wt\% Te are shown in Figs. 31 and 32. The theoretical modelling [169] successfully reproduces the measured results presented in Ref. [170]. The differences in the thermal conductivity results below $350 \mathrm{~K}$ for different values of the alloy concentration parameter $x$ can be easily understood to arise from the alloy scattering of phonons. The bipolar contribution starts to become appreciable above $300 \mathrm{~K}$, but its magnitude is larger for larger $x$ values due to the corresponding change in the electronic band gap. The minimum in $\kappa_{\text {tot }}$ lies in the range $350-450 \mathrm{~K}$, depending on the $x$ value, 
(a)

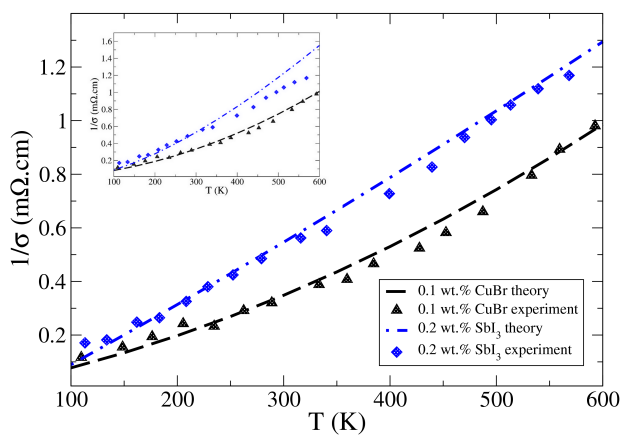

Figure 29. Temperature variation of (a) electrical resistivity and (b) electronic thermal conductivity in n-type $\mathrm{Bi}_{2}\left(\mathrm{Te}_{0.85} \mathrm{Se}_{0.15}\right)_{3}$ single crystal, containing 0.1 wt\% $\mathrm{CuBr}$ and $0.2 \mathrm{wt} \% \mathrm{SbI}_{3}$ dopants. Reproduced from [167].

as shown in the figure. In the entire range of measurement, 300-500 K, the total thermal conductivity of these p-type alloys are significantly lower than the results for the n-type samples in Fig. 30. The minimum value of $\kappa_{\text {tot }}$ is found to be $1.15 \mathrm{~W} \mathrm{~m}^{-1} \mathrm{~K}^{-1}$ for $x=0.20$ at $400 \mathrm{~K}$, which is nearly one-third of the conductivity of the n-type BiTeSe alloy presented in Fig. 30.

(a)

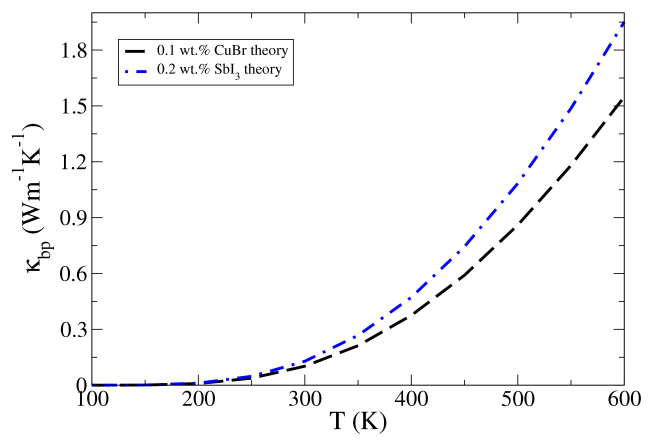

(b)

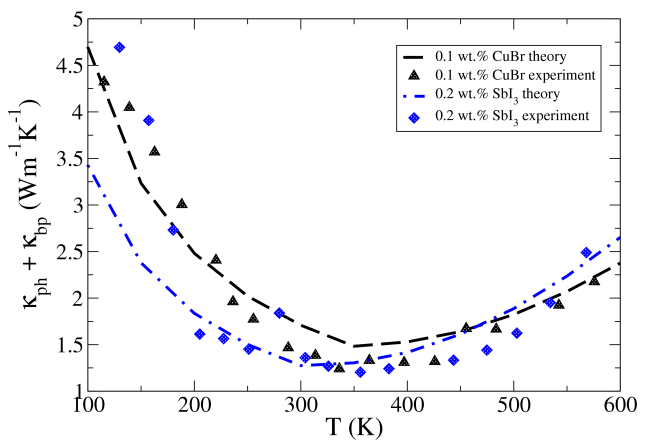

(b) total lattice thermal conductivity $\left(\kappa=\kappa_{\mathrm{ph}}+\kappa_{\mathrm{el}}\right)$ of $\mathrm{n}$-type $\mathrm{Bi}_{2}\left(\mathrm{Te}_{0.85} \mathrm{Se}_{0.15}\right)-3$ single crystal, containing $0.1 \mathrm{wt} \% \mathrm{CuBr}$ and $0.2 \mathrm{wt} \% \mathrm{SbI}_{3}$ dopants. The solid and dashed curves indicate theoretical results from Ref. [167] and the symbols represent experimental data from Ref. [168]. Taken from from Ref. [167].

The highest $Z T$ value is experimentally obtained for the for the p-type $20 \% \mathrm{Bi}_{2} \mathrm{Te}_{3}$ - $80 \% \mathrm{Sb}_{2} \mathrm{Te}_{3}$ sample as 1.33 at $398 \mathrm{~K}$. Theoretically it is found [169] that the $x=0.20$ sample has the largest $Z T$ as 1.31 at $390 \mathrm{~K}$, which is in very good agreement with the experimental value [170]. The reason that the $x=0.20$ sample has the biggest value of $Z T$ among the six compositions is two-fold: it has the lowest total thermal conductivity 
and has one of the highest power factor values. As shown in Fig. 33 the maximum $Z T$ result for this p-type sample is 2.6 times that for the n-type $\mathrm{Bi}_{2}\left(\mathrm{Te}_{0.85} \mathrm{Se}_{0.15}\right)_{3}$ single crystal doped with 0.1 wt.\% CuBr. This clearly shows that the TE figure of merit can be significantly influenced by the choice of alloy composition together with the type and amount of dopants.

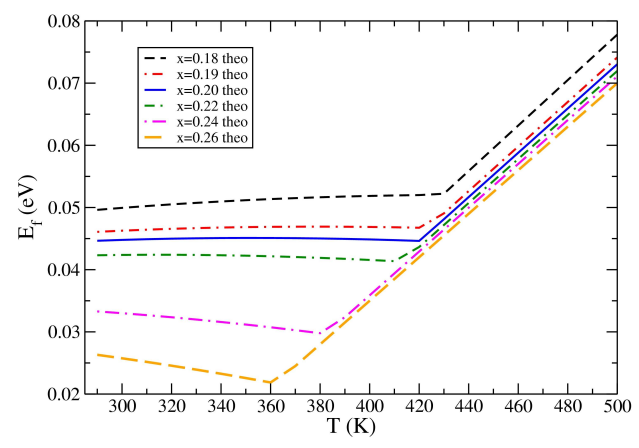

(a)

(a)

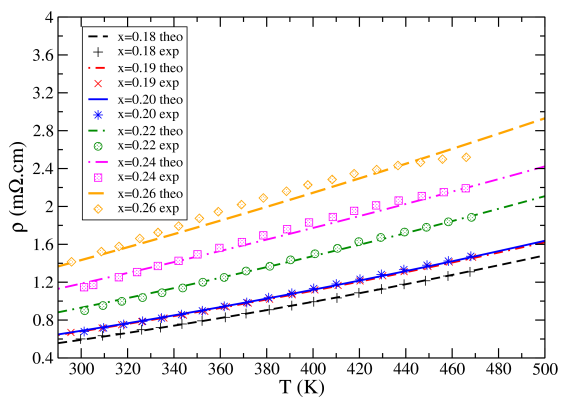

Figure 32. Temperature dependence of (a) the electrical resistivity and (b) the total thermal conductivity in p-type $\left(\mathrm{Bi}_{2} \mathrm{Te}_{3}\right)_{x}\left(\mathrm{Sb}_{2} \mathrm{Te}_{3}\right)_{1-x}$ single crystals doped with $3 \mathrm{wt} \%$ Te. Reproduced with permission from [169]. Copyright (2013), AIP Publishing LLC.

Figure 31. Temperature dependence of (a) the Fermi energy (below the conduction band minimum) and (b) Seebeck coefficient in p-type $\left(\mathrm{Bi}_{2} \mathrm{Te}_{3}\right)_{x}\left(\mathrm{Sb}_{2} \mathrm{Te}_{3}\right)_{1-x}$ single crystals doped with $3 \mathrm{wt} \%$ Te. Reproduced with permission from [169]. Copyright (2013), AIP Publishing LLC.

(b)

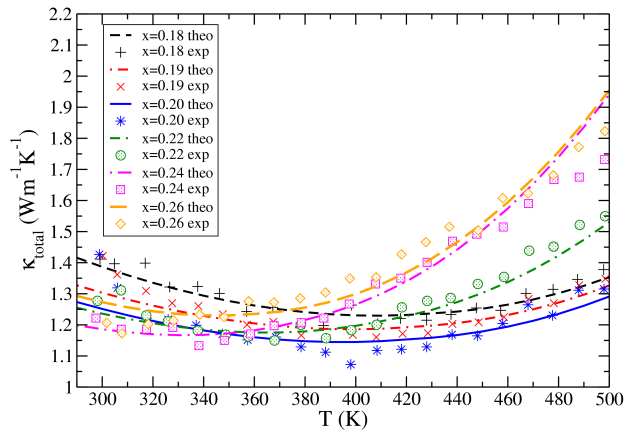

(iii) SiGe alloy nanowires and nanocompites:

Martinez et al. [171] reported the TE characteristics of individual p-type SiGe alloy nanowires for diameters of 100 to $300 \mathrm{~nm}$ and temperatures between 40 to $300 \mathrm{~K}$. Their experimental data provides evidence of the scattering of low-frequency phonons by holes of different concentrations as well as the boundary of the nanowires. The 


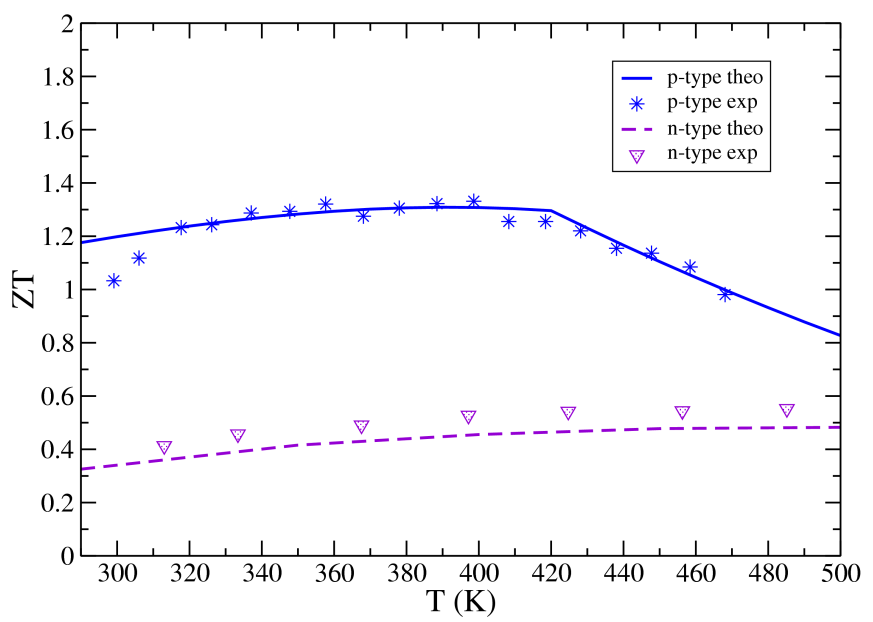

Figure 33. Temperature variation of the figure of merit for n-type and p-type BiSbTeSe alloys. Reproduced from [169].

combined boundary and hole-phonon scattering mechanisms were estimated to enhance $Z T$ by more than a factor of 2 over the bulk SiGe alloy value.

Minnich et al. [165] made a detailed theoretical investigation of the thermoelectric properties of single crystal SiGe alloys and SiGe nanocomposites. As mentioned in Sect. 6(i), after adjusting the relevant carrier and phonon scattering parameters, they successfully explained the electronic and phonon transport coefficients and the TE figure of merit $Z T$ for n- as well as p-type alloys. These authors then modelled SiGe nanocomposites by considering grain boundaries. It is known that the presence of grain boundaries reduces the electrical conductivity and can affect the Seebeck coefficient. For charge carriers, the effects of grain boundaries are usually considered by employing the charge-trapping model. The charge carriers suffer scrattering from the presence of dangling bonds and a space-charge-region with a potential barrier near the grain boundary. Minnich et al. modelled the scattering rate of charge carriers from such effects. Two distinct scattering regimes have been considered. The scattering rate of low-energy electrons follows $\tau_{\mathrm{GB}}^{-1} \propto E^{1 / 2}$, similar to the energy dependence of a diffusive boundary scattering rate. The scattering rate of high-energy electrons follows $\tau_{\mathrm{GB}}^{-1} \propto E^{-3 / 2}$, similar to the energy dependence of the ionised impurity-scattering rate. These authors also examined how the thermoelecric properties can be affected by grain boundaries by a reduction in lattice thermal conductivity. Rather than employ the modified effective medium formulation for composites as in Eq. (139), they used a standard boundary scattering rate. Accordingly, the phonon scattering rate was expressed as $\tau_{\mathrm{GB}}^{-1}=c_{s} / L_{\mathrm{GB}}$, where $L_{\mathrm{GB}}$ is atypical grain boundary size in the range 10-20 nm. No major effect of grain boundary formation was noted for the Seebeck coefficient. However, both the electronic conductivity and the phonon conductivity were 
found to reduce with the incorporation of grain-boundary scatterin rate. The difference between the conductivities with and without grain-boundary scattering decreases as the temperature increases. The phonon grain-boundary scattering rate was found to be much larger than the other phonon scattering rates over the entire temperature range. Thus, while the nanocomposite electronic conductivity approaches that of the bulk at high teperatures, the phonon conductivity remains much lower than that of the bulk. This difference in the temperature variation of the electronic and phonon conductivities leads to a net increase in $Z T$ which is most pronounced at high temperatures.

\section{(iv) Superlattice structures:}

A. General considerations and experimental results:

In bulk materials, values of $Z T$ larger than 1 have been largely achieved due to the reduction in $\kappa_{\text {ph }}$ resulting from phonon scattering by mass disorder in alloys. Complex cage-like bulk structures, such as skuterrudite, have been simulated as a phonon-glass electron-crystal (PGEC), with a view to reducing $\kappa_{\mathrm{ph}}$ without a deterioration of the electronic mobilities. However, such structures do not produce $Z T$ values much larger than 1. From these results, Venkatasubramanian et al. [172] made the observation that (i) a one-to-one correlation between lower $\kappa_{\text {ph }}$ and enhanced $Z T$ had not been established and (ii) enhancement of $Z T$ by 'tuning' phonon properties, but without producing a deterioration of electronic transport, had not been established. Thin-film materials and nanocomposite structures offer greater degree of flexibility for $Z T$ enhancement. Using such materials, several routes have been attempted. These include: (i) quantum-dot structures for obtaining an enhanced carrier density of states near the Fermi energy, (ii) phonon-blocking/electron-transmitting superlattice structures (using acoustic mismatch between the SL components to reduce $\kappa_{\mathrm{ph}}$ and potentially eliminating alloy scattering of carriers) and (iii) using thermionic effects in heterostructures. Venkatasubramanian et al. [172] demonstrated the effectiveness of route (ii). They produced high-quality ptype $\mathrm{Bi}_{2} \mathrm{Te}_{3} / \mathrm{Sb}_{2} \mathrm{Te}_{3}$ superlattices of period size $60 \AA(10 \AA / 50 \AA, 20 \AA / 40 \AA$, and $30 \AA / 30 \AA$ layer thickness in a period). Such ultra-short-period SLs offered significantly higher inplane carrier mobilities than BiSbTe alloys, owing to the near absence of carrier-alloy scattering and random carrier-interface scattering. They found that $\kappa_{\mathrm{ph}}$ is relatively independent of the SL period and the thickness of the constituents, whereas the carrier transport was dependent on the period and the relative thickness of the constituents. The room-temperature phonon conductivity $\kappa_{\mathrm{ph}}$ was measured to be $0.22 \mathrm{Wm}^{-1} \mathrm{~K}^{-1}$, much lower than the alloy results presented in Figs. 30 and 32. Uisng temperaturedependent measurements of $\sigma, S$ and $\kappa_{\text {tot }}$, they obtained $Z T$ vs. T results shown in Fig. 34 for the $10 \AA / 50 \AA$ SL. The room-temperature value of $Z T \sim 2.4$ was estimated. Their results indicate that phonon and hole (charge carriers) transport can be fine-tuned to improve $Z T$.

B. Computational results mainly focussed on electronic components:

Using the effective mass theory and the CRTA (cf. Sec. 3.2) for calculating the electronic TE coefficients $S, \sigma$ and $\kappa_{\text {el }}$, but treating $\kappa_{\mathrm{ph}}$ as an adjustable parameter, 


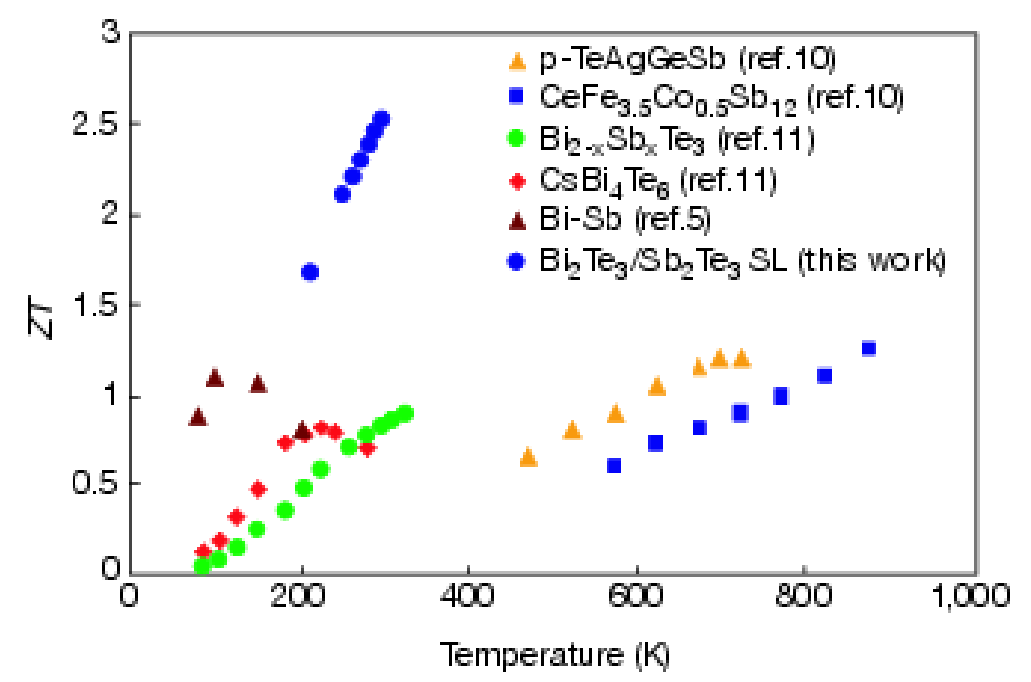

Figure 34. Temperature variation of the figure of merit for n-type and p-type $\mathrm{Bi}_{2} \mathrm{Te}_{3}(10 \AA) / \mathrm{Sb}_{2} \mathrm{Te}_{3}(50 \AA)$ superlattice. Results for some other materials have also been presented. Reproduced from [172].

Hicks and Dresselhaus [44] proposed that it may be possible to increase ZT of certain materials by preparing them in quantum-well superlattice structures. Using their simple theoretical scheme, they indicated that $\mathrm{Bi}_{2} \mathrm{Te}_{3}$ in a quantum-well structure has the potential to increase $Z T$ by an order of magnitude over the bulk value. Their theory includes the electron confinement effect due to quantum well formation. However, it treats the phonon conductivity $\kappa_{\mathrm{ph}}$ as a simple adjustable parameter, without providing any basis for that. Since, as discussed in the preceeding section, it is expected that $\kappa_{\mathrm{ph}}$ would change (reduce) significantly and thus its adjusted parametric value should also change upon quantum well formation, it is difficult to assess from the results presented in [44] the roles of electron confinement and phonon tuning in the enhancement of $Z T$.

By employing a similar theoretical method, Sofo and Mahan [173] showed that there is an improvement of the figure of merit, produced by the enhancement of the electronic density of states at the bottom of the lowest subband. This enhancement is produced by the increase of the effective mass along the superlattice growth direction. It was found that a moderate increase of $Z T$ may be expected for intermediate values of the superlattice period. However, $Z T$ starts to be reduced with further reduction of the period because of the broadening of the lowest subband, approaching a 3D-like density of states.

Lin-Chung and Reinecke [34] developed the theory of TE transport coefficents for superlattices as presented in Sec. 2. Using the effective mass theory and the CRTA for calculating the electronic TE coefficients $S, \sigma$ and $\kappa_{\mathrm{el}}$, but treating $\kappa_{\mathrm{ph}}$ as an adjustable parameter, they examined the variation of $Z T$ as a function of the ratio of well $\left(\mathrm{Bi}_{2} \mathrm{Te}_{3}\right)$ and barrier $\left(\mathrm{Pb}_{0.75} \mathrm{Sn}_{0.25} \mathrm{Te}_{3}\right)$ in a superlattice structure. Their results for cross-plane and in-plane components of $Z T$ are presented in Fig. 35. Their work recovers the results obtained by Hicks and Dresselhaus for the case when the barrier thickness is zero. It was 
found that the cross-plane component of ZT lies between the results for the bulks $\mathrm{Bi}_{2} \mathrm{Te}_{3}$ and $\mathrm{Pb}_{0.75} \mathrm{Sn}_{0.25} \mathrm{Te}_{3}$. It was also found that for certain barrier/well width ratios the inplane component of $Z T$ can become much larger than the higher of the constituent bulk values. Similar to the work of Hicks and Dresselhaus [44], these authors also treated the phonon conductivity $\kappa_{\mathrm{ph}}$ as a simple adjustable parameter, without providing any basis for that. Therefore, while valuable information can be extracted regarding the effect of the barrier/well width ratio for changes in $Z T$, no information can be gained with regards to the role of phonon tuning.

(a)

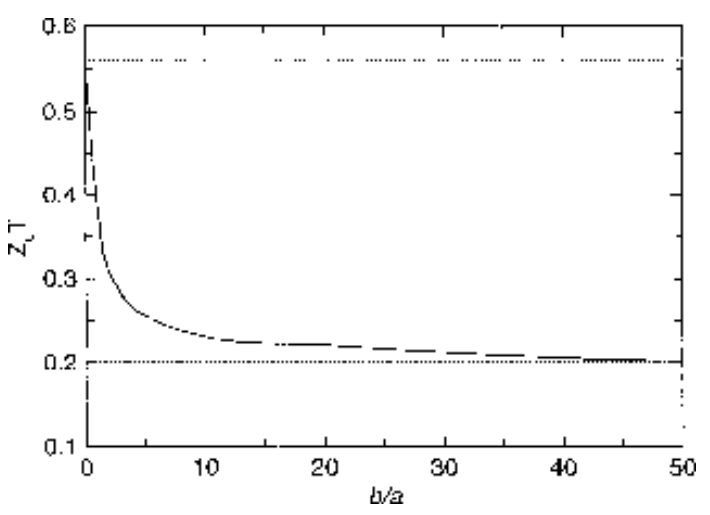

(b)

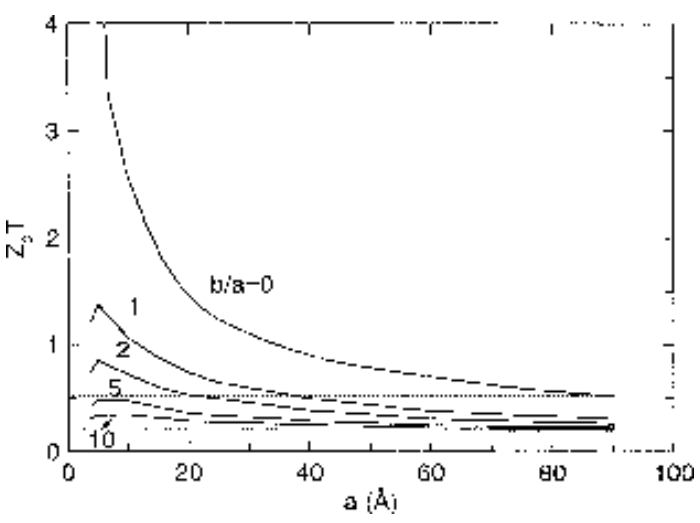

Figure 35. Variation of $Z T$ as a function of the barrier/well width ratio for the $\mathrm{Bi}_{2} \mathrm{Te}_{3} / \mathrm{Pb}_{0.75} \mathrm{Sn}_{0.25} \mathrm{Te}_{3}$ superlattice: (a) when current flows along the growth direction (cross-plane $Z T$ ) and (b) when current flows perpendicular the growth direction (inplane $Z T)$. Reproduced from [34].

Anisotropy and non-parabolicity in the electronic band structure may play a big role in estimating the electronic TE transport coefficients of certain semiconductors, such as PbTe [174, 175, 176]. However, Broido and Reinecke [175], using (i) full superlattice electronic band structure and (ii) a multiband inelastic Boltzmann equation for carrierphonon scattering, presented a less optimistic view than the results obtained by Hicks and Dresselhaus [44] and Lin-Chung and Reinecke [34] from the use of the simple effective mass (i.e. parabolic bands) theory and the CRTA. Broido and Reinecke found that the increased carrier scattering rates that occur with increasing confinement in superlattice structures cause the power factor $S^{2} \sigma$ to remain near the bulk value for all barrier heights. Broido and Reinecke also suggested that similar findings are expected for all semiconductor superlattice systems. Once again, however, no information can be gained from this work regarding the role of phonon tuning in the enhancement of $Z T$.

Based upon an analysis of the theoretical investigations and the experimental results presented so far in this section, it becomes clear that the 'tuning' of phonon properties is likely to play a more significant role in obtaining enhancement in $Z T$ of superlattice systems, and of nanomaterials in general. This is supported by the recent experimental work of Zhang et al. [177] whose TE property measurements indicate that enhancement in $Z T$ for n-type $\mathrm{Bi}_{2} \mathrm{Te}_{3}$ nanowire samples results from significantly reduced thermal conductivity due to enhanced phonon scattering at the nanoscale in- 
terfaces.

C. Computational results focussed on phonon tuning:

( $\alpha$ ) $\mathrm{Bi}_{2} \mathrm{Te}_{3}$ quantum well structures:

Some theoretical effort has been made to examine the role of phonon confinement towards an enhancement of the TE figure of merit of nanostructures. Balandin et al. [178] studied the effect of phonon confinement on the enhancement of $Z T$ for $\mathrm{Bi}_{2} \mathrm{Te}_{3}$ quantum wells. These authors modelled the confinement of acoustic phonons by employing the continuous medium approximation for quantum well structures with freesurface boundaries. They treated the electronic contributions using a scheme similar to that employed by Hicks and Dresselhaus [44]. This study suggests that at room temperature the phonon confinement effect can give rise to an enhancement of $Z T$ as large as two times the value without the confinement for a $90 \AA$ wide quantum well. This suggestion has to be treated with some caution, and may not be numerically accurate. This is because the expression used in their work for anharmonic phonon ineraction is too simplistic and only valid at high temperatures. Also, this work did not consider phonon scattering from rough and defective interfaces, which may considerably change the enhancement factor for $Z T$. The role of optical phonons was completely ignored.

Theoretical investigations have recently been made [179] for the $\mathrm{Bi}_{2} \mathrm{Se}_{3} / \mathrm{Bi}_{2} \mathrm{Te}_{3} / \mathrm{Bi}_{2} \mathrm{Se}_{3}$ and $\mathrm{Sb}_{2} \mathrm{Te}_{3} / \mathrm{Bi}_{2} \mathrm{Te}_{3} / \mathrm{Sb}_{2} \mathrm{Te}_{3}$ quantum well systems, with $\mathrm{Bi}_{2} \mathrm{Te}_{3}$ considered as the well material. Calculations were made by employing the Hicks-Dresselhaus scheme for the electronic transport coefficients and the Lin-Chung-Reinecke scheme for the thermoelectric transport coefficients of superlattice structures (as described in Sect. 2). The phonon conductivity was calculated by employing an isotropic continuum scheme for both the harmonic and anharmonic forces (as described in Sects. 4.3.2 and 4.4). The phonon-interface scattering due to the mass-smudging effect was incorporated by employing a simplified version of the theory described in Sect. 4.4.2: in particular, the probability of layer mixing was chosen to decrease exponentially from an interface. The anharmonic phonon relaxation rate in the quantum well structures was considered, in a very simplified manner following Ref. [180], of the form

$$
\tau_{\boldsymbol{q}_{s}}^{-1}(\mathrm{anh}, \mathrm{QW})=\tau_{\boldsymbol{q}_{s}}^{-1}(\mathrm{anh}, \mathrm{bulk})\left(n_{A}+n_{B}\right)^{2 / 3},
$$

where $n_{A}$ and $n_{B}$ are the numbers of atomic layers of the well and barrier materials, respectively, and Eq. (125) is used for $\tau_{\boldsymbol{q}_{s}}^{-1}$ (anh, bulk). Although the ad hoc choice of the expressions used in this work for the interface and anharmonic scatterings of phonons may be subject to scrutiny, it is useful to note that this investigation made the following conclusions. (a) For the n-type quantum well of width $d_{A}=7 \mathrm{~nm}$ the maximum value of the power factor is an order of magnitude larger than its bulk value given in Ref. [167]. The highest value of the power factor for the p-type quantum well sample with $d_{A}=10$ $\mathrm{nm}$ is nearly four times bigger than its bulk value obtained in Ref. [169]. (b) An order of magnitude smaller values of $\kappa_{\mathrm{ph}}$ are predicted for almost all the considered quantum well systems compared to their bulk forms. (c) The largest $Z T$ value is predicted to 
be 0.97 at $350 \mathrm{~K}$ for the n-type quantum well system of $d_{A}=7 \mathrm{~nm}$ and 1.945 at $440 \mathrm{~K}$ for the p-type quantum well material of $d_{A}=10 \mathrm{~nm}$. Essentially, therefore, this work suggests that the enhancement of $Z T$ is almost equally contributed by the electronic and phonon transport coefficients upon the quantum well formation.

\section{( $\beta$ ) Ultra-thin SiGe superlattices:}

Recently, calculations were performed [181] of the electronic and phonon transport coefficients of an n-type $\mathrm{Si}_{4} \mathrm{Ge}_{4}[001]$ superlattice, with each unit cell containing 4 atomic bilayers of Si and 4 bilayers of Ge. For such a system, following the theoretical prediction in [182], the Si layers were considered as quantum wells and the Ge layers as quantum barriers. The electronic transport coefficients $\left(S, \sigma\right.$ and $\left.\kappa_{\text {carriers }}\right)$ were computed using the theory described in Sect. 2. A single electron confined state was considered in the Si quantum well. When calculating $\sigma$ the dominant scattering process was assumed to be the acoustic phonon scattering. The phonon dispersion relations and phonon velocity components were computed from the application of the DFPT scheme - the dispersion curves along two symmetry directions are presented in the lower panel of Fig. 9. A finite superlattice length of $0.2 \mu \mathrm{m}$ was taken as an effective phonon boundary scattering length. The mass-mixing in the first and second bilayers was modelled with the probabilities of 0.05 and 0.0025 , respectively.

Figure 36 shows the phonon conductivity results along and across the superlattice growth direction. The in-plane values are much larger than the cross-plane values: about 6 times larger at $300 \mathrm{~K}$. A comparison with Fig. 26 shows that for the considered probabilty of interface mass mixing, the cross-plane conductivity of the SL is much lower than that of the n-type $\mathrm{Si}_{0.754} \mathrm{Ge}_{0.246}$ alloy. Figure 37 presents the calculated results for the power factor $\sigma S^{2}$ and the figure of merit $Z T$, with a doping concentration of $9.4 \times 10^{25}$ $\mathrm{m}^{-3}$. Both the in-plane and cross-plane components of the power factor are an order of magnitude or so greater than that of the alloy. The in-plane component of the figure of merit $Z T$ is larger than the in-plane componet and greater than the alloy value. This is a significant difference from the result presented by Lin-Chung and Reinecker [34]. This difference can be ascribed to a more accurate calculation of the phonon conductivity in [181]. The results in Fig. 37 suggest that, depending on the interface quality, it may be possible to obtain an enhancement of $Z T$ beyond 3 at temperatures above $1000 \mathrm{~K}$. Such an enhancement of $Z T$ for ultra-thin superlattices with reasonably mass-smudged interfaces would definitely be far superior to that for SiGe alloy nanocomposites suggested in [165].

\section{(v) Important factors for tuning TE transport coefficients:}

Although many more systematic studies, preferably employing first-principles approaches, are required to make numerical assessment of the relative roles of important factors for tuning TE transport coefficients, it is possible to draw some conclusions from the results presented in this section. The carrier effective mass and confinement effects are governed by the chemical composition of the well and barriers materials. Several 


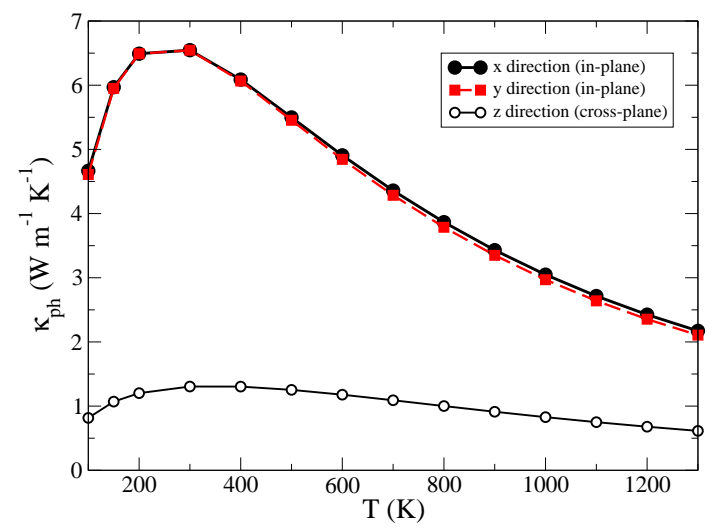

Figure 36. In-plane and cross-plane components of the phonon conductivity of the $\mathrm{Si}_{4} \mathrm{Ge}_{4}[001]$ superlattice. Reproduced with permission from [181]. Copyright (2014), AIP Publishing LLC.

(a)
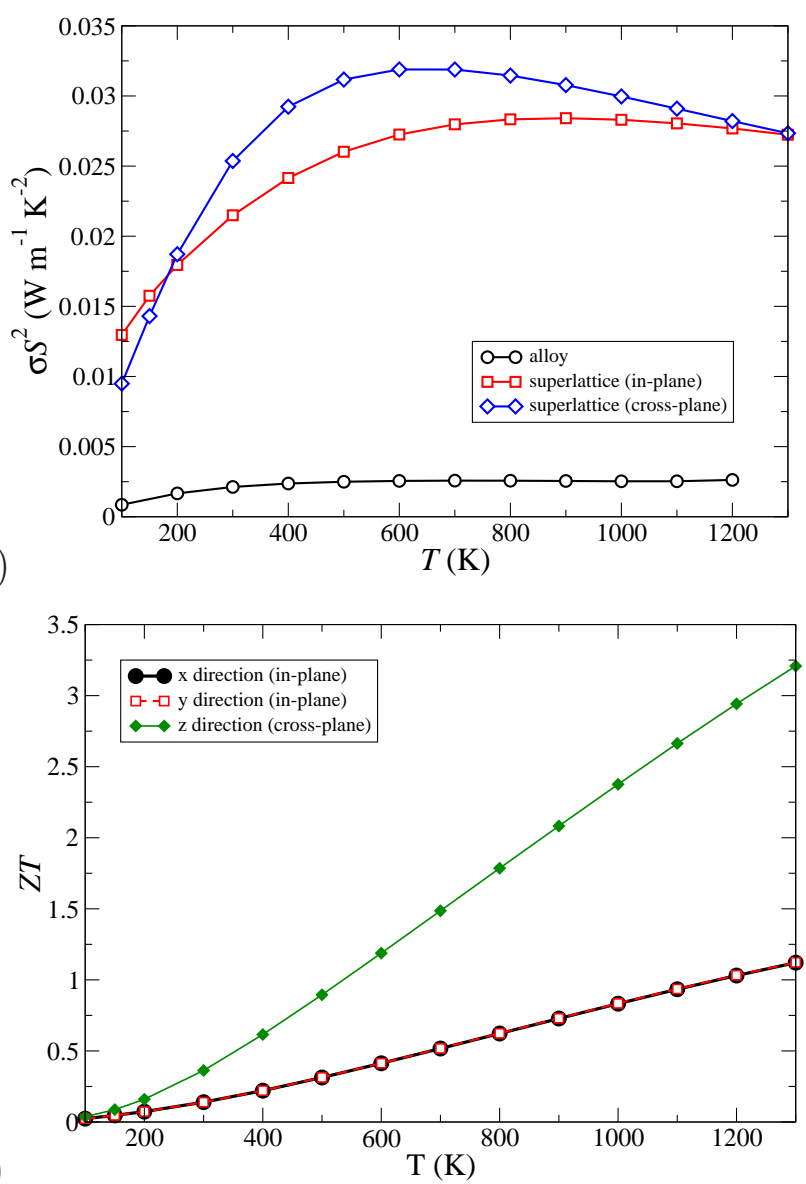

(b)

Figure 37. Computed results of (a) power factor, (b) figure of merit for the $\mathrm{Si}_{4} \mathrm{Ge}_{4}[001]$ superlattice. The results are also compared with those for the $\mathrm{Si}_{x} x \mathrm{Ge}_{x} x$ alloy. Reproduced with permission from [181]. Copyright (2014), AIP Publishing LLC.

rather obscure factors play roles of different degrees of effectiveness in controlling phonon transport in low-dimensional systems, particlualy in nanocomposites. These include: 
grain boundary size, mass-disorder, volume fraction of nanoparticle in the host matrix, interface scattering, and the degree of disorder at interfaces. For an optimistic level of enhancement for $Z T$, these factors should be controlled so as to achieve the PGEC-like behaviour of the nanocomposite structure.

\section{Summary and future outlook}

In this review article we have presented a summary of the theoretical progress made towards our understanding of the thermoelectric properties of semiconducting materials. Both phenomenological and $a b$ initio levels of theoretical developments have been covered. Analysis of available experimental and theoretical results suggests that the thermoelectric figure of merit $Z T$ can be enhanced much more effectively by 'tuning' phonon properties via fabrication of nanocomposites with rough interfaces. A theory of the phonon scattering rate due to mass-smudging and broken bonds at superlattice interfaces has been discussed in some detail. Preliminary computational results suggest that $Z T>3$ can be achieved over a wide range of temperatures in ultrathin $\mathrm{Si} / \mathrm{Ge}$ superlattices fabricated with reasonably rough (mass-smudged) interfaces. These results reassure us of the optimism expressed in the Introduction of achieving $Z T>4$ for suitably doped semiconductor nanocomposite structures with rough interfaces.

There is great need for gaining a firm grip over producing controlled chemical and structural properties of interfaces, and for an accurate numerical assessment of their phonon scattering strengths. With these caveats, there is great potential for extending the current level of theoretical development for the lattice thermal conductivity of nanocomposite materials, such as superlattices, dispered nanowires in a host matrix, dispersed nanodots in a host matrix, etc.

Several research groups are actively involved with accurate, first-priciples, calculations of $\kappa_{\mathrm{ph}}$. Such effort will produce reliable results with predictive power for $\kappa_{\mathrm{ph}}$ of nanostructures with sharp, unmixed, interfaces. However, there remain two major challenges that must be overcome for the theoretical/computational progress to become useful for enahmcement in $Z T$. The first point is that in most materials the third-order anharmonic force constants are highly temperature dependent. This can be appreciated by examining measurements of the temperature-dependent Grüneisen constant for such materials. Such a consideration would be important for obtaining accurate honon conductivity results above the Debye temperature. However, at present there are no clear theoretical guidelines for the inclusion of temperature dependence in first-principles calculations of anharmonic force constants, and at best some ad hoc schemes must be adopted. The second point is related to phonon-interface scattering. Such scattering becomes more effective for thin nanomaterials. Unfortunately, it is difficult to control the quality of interfaces during nanocomposite formation. This would make a theorist's job extremely difficult to develop a definite theory for phonon-interface scatterings. The best one can hope for is that a number of practical considerations can be made in theoretical modelling and useful guidelines provided for the control in phonon 
lifetime due to interface scattering.

On the whole, the rapid rate of experimental and theoretical developments in the investigation of both carrier transport coefficients $\left(S, \sigma\right.$ and $\left.\kappa_{\text {carrier }}\right)$ and the lattice thermal transport coefficient $\kappa_{\mathrm{ph}}$ is encouraging and will prove beneficial in the development of technologically feasible thermoelectric materials. Hopefully, theory and experiment will work together and complement each other to 'tune' phonon properties effectively for a reliable degree of achievement of enhancement in $Z T$.

\section{Acknowledgements}

I would like to thank my postgraduate students, postdoctoral fellows and research collaborators who have helped me develop the ideas presented in this article. In particular, I would like to thank Ceyda Yelgel for her contribution in the initial development of the TE project, and Steven Hepplestone and Iorwerth Thomas for their contribution in the development of phonon-interface and anharmonic interactions in superlattice structures presented in this review article. I also thank Steve and Iorwerth for a careful reading of this manuscript and making suggestions for improvement of the material presented. Finally, I express my sincere thanks to the EPSRC (UK) for their support of our research on phonon engineering of nanocomposite thermoelectric materials through the grant number EP/H046690.

\section{References}

[1] Ioffe A F 1957 Semiconductor, Thermoelements and Thermoelectric Cooling (London: Infosearch)

[2] Drabble J R and Goldsmid H J 1961 Thermal Conduction in Semiconductors (Oxford: Pergamon Press)

[3] Ure R W and Heikes R R (Eds.) 1961 Thermoelectricity: Science and Engineering (New York: Interscience)

[4] Goldsmid H J 1965 The Thermal Properties of Solids (New York: Dover Publications)

[5] Rowe D M and Bhandari C M 1983 Modern Thermoelectrics (Virginia: Reston Publishing Company, Inc.)

[6] Tritt T M (Ed.) 2001 Recent Trends in Thermoelectric Materials Research I, II, III (San Diego: Academic Press)

[7] Rowe D M 2006 Thermoelectrics Handbook (Boca Raton: Taylor and Francis Group)

[8] Zlatic V and Hewson A C (Eds.) 2009 Properties and Applications of Thermoelectric Materials (Dordrecht: Springer)

[9] Goldsmid H J 2010 Introduction to Thermoelectricity (Heidelberg: Springer-Verlag)

[10] Ioffe A F and Stil'bans 1959 Rep. Prog. Phys. 22167

[11] Rosi F D 1968 Solid State Electronics 11833

[12] Wright D A 1970 Metallurgical Reviews 15147

[13] Yim W M and Rosi F D 1972 Solid State Electronics 141121

[14] Wood C 1984 Energy Convers. Mgmt 24317

[15] Vining C B 1992 Proc. XI International Conference on Thermoelectrics (University of Texas at Arlington, Texas)

[16] Mahan G D (1989) J Appl Phys 651578

[17] DiSalvo F J 1999 Science 285703 
[18] Chen G, Dresselhaus M S, Dresselhaus G, Fleurial J-P and Caillat T 2003 International Materials Reviews 4845

[19] Rowe D M 2008 International Journal of Innovations in Energy Systems and Power 113

[20] Zheng J-C 2008 Front. Phys. China 3269

[21] Bulusu A and Walker D G 2008 Superlattices and Microstructures 441

[22] Pichanusakorn P and Bandaru P 2010 Materials Sci Engg R 6719

[23] Wan C, Wang Y, Wang N, Norimatsu W, Kusunoki M and Koumoto K 2010 Sci Technol Adv Mater 11044306

[24] Vineis C J, Shakouri A, Majumdar A and Kanatzidis M G 2010 Adv Mat 223970

[25] Liu W, Yan X, Chen G and Ren Z 2012 Nanoenergy 142

[26] Maldovan M 2013 Nature 503209

[27] Goldsmid H J 1960 Br. J. Appl. Phys. 11209

[28] Leibfried G and Schlömann E 1954 Nachr. Akad. Wiss., Göttingen, Kl. 2, Math-Physik 2, 71

[29] Keyes R W 1959 Phys Rev 115564

[30] Moralli D T and Slack G A 2006 in High Thermal Conductivity Materials, Eds. S L Shindé and J S Goel (Springer, New York)

[31] Meddins H R and Parrott J E 1976 J. Phys. C: Solid State Phys. 9, 1263

[32] Slack G 1994 in CRC Handbook of Thermoelectrics, Ed. by D. M. Rowe (CRC Press, Boca Raton), p. 407

[33] Bergman D J and Levy O 1991 J Appl Phys 706821

[34] Lin-Chung P J and Reinecke T L (1995) Phys. Rev. B 51, 13244

[35] Ziman J M Electrons and Phonons (Oxford: Clarendon Press)

[36] Hinsche1 N F, Mertig I and Zahn P 2012 J Phys Condens Matter 24275501

[37] Press W H, Flanney B P, Teukolsky S A and Vetterling W T 1986 Numerical Recipies: The Art of Scientific Computing (Cambridge University Press, Cambridge)

[38] Blakemore J S 1962 Semiconductor Statistics (Pergamon Press, Oxford)

[39] McKelvey J P 1966 Solid State and Semiconductor Physics (International Edition) (Harper and Row, New York, Evanston, London, and John Weatherhill Inc., Tokyo)

[40] Hutner R A, Rittner E S and DuPrè F K 1950 Phillips Research Reports 5, 188

[41] Nag B R 1972 Theory of Electrical Transport in Semiconductors (Pergamon, Oxford)

[42] Ridley B K 1982 J. Phys. C: Solid State Phys.15, 5899

[43] Drabble J R and Goldsmit H J 1961 Thermal Conduction in Semiconductors (Pergamon, Oxford)

[44] Hicks L D and Dresselhaus M S 1993 Phys Rev B 4712727

[45] Hicks L D and Dresselhaus M S 1993 Phys Rev B 4716631

[46] Broido D A and Reinecke T L 1997 Appl Phys Lett 702834.

[47] Price P J 1955 Phil Mag 461192

[48] Srivastava G P 1990 The Physics of Phonons (Adam Hilger, Bristol, - now Taylor and Francis Group).

[49] Srivastava G P 1999 Theoretical Modelling of Semiconductor Surfaces (World Scientific, Singapore).

[50] Baroni S, de Gironcoli S, Dal Corso A and Giannozzi P 2001 Rev Mod Phys 73515

[51] Weber W 1977 Phys Rev B 154789

[52] Stillinger F H and Weber T A (1985) Phys Rev B 315262

[53] Tersoff J (1988) Phys Rev B 389902

[54] Harrison W (1989) Electronic Structure amd the Properties of Solids (Dover, New York, 1989)

[55] Bazant M Z, Kaxiras E and Justo J F (1997) Phys Rev B 568542

[56] Justo J F, Bazant M Z, Kaxiras E, Bulatov V V and Yip S (1998) Phys Rev B 582539

[57] Sood A K, Menèndez J, Cardona M and Ploog K (1985) Phys Rev B 542111

[58] Lambert K and Srivastava G P (1997) Phys Rev B 5613387

[59] Mingo N and Yang L (2003) Phys Rev B 68245406

[60] Stroscio M A and Dutta M 2001 Phonons in Nanostructures (Cambridge University Press, 
Cambridge).

[61] Born M 1914 Ann Phys Lpz 44605

[62] Born M and Huang K 1954 Dynamical Theory of Crystal Lattices (Oxford University Press, Oxford).

[63] Musgrave M J P and Pople J A 1962 Proc R Soc A 268474

[64] Keating P N 1966 Phys Rev 145637

[65] Martin R M 1970 Phys Rev B 14005

[66] Kellerman E W 1940 Phil Trans R Soc A 238513

[67] Banerjee R and Varshni Y P 1969 Can J Phys 47451

[68] Vetelino J F and Mitra S S 1969 Phys Rev 1781349

[69] Dick B G and Overhauser A W 1958 Phys Rev 11290

[70] Cochran W 1959 Proc R Soc A 253260

[71] Waugh J L and Dolling G 1963 Phys Rev 1322410

[72] Schröder U 1966 Solid State Commun 4347

[73] Kress W 1972 phys stat sol (b) 49239

[74] Phillips J C 1968 Phys Rev 166832

[75] Weber W 1974 Phys Rev Lett 33371

[76] Rustagi K C and Weber W 1976 Solid State Commun 18673

[77] Giannozzi P, de Gironcoli S, Pavone P and Baroni S 1991 Phys Rev B 437231

[78] Monkhorst H J and Pack J D 1976 Phys Rev B 13, 5189

[79] Chadi D J and Cohen M L 1973 Phys Rev B 85747

[80] Tütüncü H M 1997 Lattice Dynamics of Semiconductors and Their Surfaces (Ph D thesis, University of Exeter, UK).

[81] Warren J L, Yarnell J L, Dolling G and Cowley R A 1967 Phys Rev 158805

[82] Price D L, Rowe J M and Nicklow R N 1971 Phys Rev B 31268

[83] Nilsson G and Nelin G 1971 Phys Rev B 3364

[84] Nilsson G and Nelin G 1972 Phys Rev B 63777

[85] Hepplestone S P and Srivastava G P 2006 Nanotechnol 173288

[86] Thonhauser T and Mahan G D 2004 Phys Rev B 69075213

[87] Nishiguchi N, Ando Y and Wybourne M N 1997 J Phys: Cond Matter 95751

[88] Li J C, Wang C L, Peng H, Wang M X, Zhang R Z, Wang H C, Liu J, Zhao M L and Mei L M (2010) J Appl Phys 108063702

[89] Thomas I O and Srivastava G P 2013 Phys Rev B 87085410

[90] Thomas I O and Srivastava G P 2013 Phys Rev B 88115207

[91] Ezzahri Y, Grauby S, Rampnoux J M, Michel H, Pernot G, Claeys W, Dilhaire S, Rossignol C, Zeng G and Shakouri A 2007 Phys Rev B 75195309

[92] Hepplestone S P and Srivastava G P 2008 Phys Rev Lett 101105502

[93] Kubo R 1957 J Phys Soc Japan 12570

[94] McGaughey A J H and Kaviany M 2004 Phys Rev B 69094303

[95] Kaburaki H, Yip S and Kimizuka H 2007 J Appl Phys 102043514

[96] Huang B-L and Kaviany M 2008 Phys Rev B 77125209

[97] Ziman J M 1969 Elements of Advanced Quantum Theory (Cambridge University Press, Oxford)

[98] Hamilton R A H and Parrott J E 1969 Phys Rev 1781284

[99] Peierls R 1935 Ann. Inst. H Poincaré 5177

[100] Klemens P G 1951 Proc R Soc A 208108

[101] Guyer R A and Krumhansl J A 1966 Phys Rev 148766

[102] Benin D 1970 Phys Rev B 12777

[103] Srivastava G P 1976 J Phys C: Solid State Phys 93037

[104] G. P. Srivastava G P 1976 J Phys C: Solid State Phys 101843

[105] Arthurs A M 1970 Complementary Variational Principles (Clarendon, Oxford)

[106] Srivastava G P and Hamilton R A H 1978 Phys Rep 38 C 1 
[107] Carruthers P 1961 Rev Mod Phys 3392

[108] Callaway J 1959 Phys Rev 1131046

[109] Hamilton R A H 1973 J Phys C 62653

[110] Simons S 1975 J Phys C 81147

[111] Srivastava G P 1976 Phil Mag 34795

[112] Parrott J E 1971 phys stat sol (b) 48 K159

[113] Omini M and Sparavigna A 1997 Nuovo Cimento D 191537

[114] Broido D A, Ward A and Mingo N 2005 Phys Rev B 72014308

[115] Holland M G 1964 Phys Rev 134, A471

[116] Ziman J M (1956) Phil Mag 1191

[117] Ziman J M (1957) Phil Mag 2292

[118] Parrott J E (1979) Rev Int Hautes Tem Refract 16393

[119] Garg J, Bonini N and Marzari N 2014 Ch 4 in Length-scale Dependent phonon Interactions (eds. S L Shind'e and G P Srivastava, Springer, New York)

[120] Mingo N, Stewart D A, Broido D A, Lindsay L and Li W 2014 Ch 5 in Length-scale Dependent phonon Interactions (eds. S L Shind'e and G P Srivastava, Springer, New York)

[121] Klemens P G 1966 Phys Rev 148845

[122] Srivastava G P 1980 J Phys Chem Solids 41357

[123] Thomas I O and Srivastava G P 2012 Phys Rev B 86045205

[124] Klemens P G 1958 in Solid State Physics Vol.7 (eds. F Seitz and D Turnbull, Academic Press, New York), p. 1.

[125] Ridley B K and Gupta R 1990 Phys Rev 434939

[126] Ren and Dow 1982 Phys Rev B 253750

[127] Hepplestone S P and Srivastava G P 2010 Phys Rev B 82144303

[128] Hepplestone S P and Srivastava G P 2011 Phys Rev B 84115326

[129] Landry E S and McGaughey A J H 2009 Phys Rev B 79075316

[130] Tian Z, Esfarzani K and Chen G 2012 Phys Rev B 86235304

[131] Yin C F and Truell J (1956) J Appl Phys 271086

[132] Kim W and Majumdar A (2006) J Appl Phys 99084306

[133] Minnich A J and Chen G (2007) Appl Phys Lett 91073105

[134] Gillet J-N, Chalopin Y and Volz S (2009) J Heat Transfer 131043206

[135] Behrang A, Grmela A M, Dubois C, Turenne S and Lafleur P G (2013) J Appl Phys 114014305

[136] Inyushkin A V, Taldenkov A N, Gibin A M, Gusev A V and Pohl H-J (2004) phys stat solidi (c) 12995

[137] Ozhogin V I, Inyushkin A V, Taldenkov A N, Tikhomirov A V and Popov G E (1996) JETP Lett 63490

[138] Holland M G and Neuringer L J (1962) Proc. Int. Congr. on the Physics of Semiconductors, Exeter (London: Institute of Physics) p 475

[139] Geballe T H and Hull G W (1958) Phys Rev 110773

[140] Vining C B (1991) J Appl Phys 69331

[141] Meddins H R and Parrott J E (1976) J Phys C: Solid State Phys 91263

[142] Garg J, Bonini N, Kozinsky B and Marzari N (2011) Phys Rev Lett 106045901

[143] Abeles B, Beers D S, Cody G D and Dismukes J P (1962) Phys Rev 12544

[144] Capinski W S, Maris H J, Ruf T, Cardona M, Ploog K and Katzer D S (1999) Phys Rev B 59 8105

[145] Swartz E T and Pohl R O (1989) Rev Mod Phys 61605

[146] Cahill D G, Katiyar M and Abelson J R (1994) Phys Rev B 506077

[147] Lee S-M, Cahill D G and Venkatasubramanian R (1997) Appl Phys Lett 702957

[148] Borca-Tasciuc T, Liu W, Zeng T, Song D W, Moore C D, Chen G, Wang K L, Goorsky M S, Radetic T, Gronsky R, Koga T and Dresselhaus M S (2000) Superlattices and Microstructures 28199 
[149] Huxtable S T, Abramson A R, Tien C-L, Majumdar A, LaBounty C, Fan X, Zeng, G and Bowers J E (2002) Appl Phys Lett 801737

[150] Huxtable S T, Abramson A R, Majumdar A, Shakouri A, Croke E T (2002) ASME International Mechanical Engineering Congress and Exposition, IMECE2002-34239, pp 1-5

[151] Venkatasubramanian R and Colpits T (1997) Mater Res Soc 47873

[152] Garg J, Bonini N and Marzari N (2011) Nano Lett 115135

[153] Yates B Thermal Expansion (New York, Plenum, 1972)

[154] Gauster W B (1973) J Appl Phys 441089

[155] Glassbrenner C J and Slack G A (1964) Phys Rev 134 A1058

[156] Li D, Wu Y, Kim P, Shi L, Yang P and Majumdar A (2003) Appl Phys Lett 832934

[157] Walkauskas S G, Broido D A, Kempa K and Reinecke T L (1999) J Appl Phys 852579

[158] Voltz S G and Chen G (1999) Appl Phys Lett 752056

[159] Murphy P G and Moore J E (2007) Phys Rev B 76155313

[160] Srivastava G P (2009) Mater Res Soc Symp Proc 11732 1172-T08-07

[161] Yang R, Chen G and Dresselhaus M S (2005) Phys Rev B 72125418

[162] Mingo N, Hauser D, Kobayashi N P, Plissonnier M and Shakouri A 2009 Nano Lett 9711

[163] Behrang A, Grmela M, Dubois C, Turenne S, Lafleur P G and Lebon G 2014 Appl Phys Lett 104063106

[164] Theoretical/computational groups actively involved in first-principles calculations of the phonon conductivity of bulk and nanostructured materails include, among others: Chen's group at MIT; Marzari's group at Lausanne; Broido's group at Boston; Mingo's group at Grenoble; Stewart's group at Cornell; Lindsay's group at Washington.

[165] Minnich A J, Lee H, Wang X W, Joshi G, Dresselhaus M S, Ren Z F, Chen G and Vashaee D 2009 Phys Rev B 80155327

[166] Steigmeier E F and Abeles B (1964) Phys Rev 136 A1149

[167] Yelgel Ö C and Srivastava G P (2012) Phys Rev B 85125207

[168] Hyun D B, Hwang J S, You B C, Oh T S and Hwang C W (1998) J Mat Sci 335595

[169] Yelgel Ö C and Srivastava G P (2013) J Appl Phys 113073709

[170] Li D, Sun R R and Qin X Y (2011) Intermet. 192002

[171] Martinez J A, Provencio P P, Picraux S T, Sullivan J P and Swartzentruber B S 2011 J Appl Phys 110074317

[172] Venkatasubramanian R, Siivola E, Colpitts T and O'Quinn B (2001) Nature 413597

[173] Sofo J O and Mahan G D (1995) Appl Phys Lett 652690

[174] Ravich Y I, Efimova B A and Smirnov I A (1970) Semiconducting Lead Chalcogenides, Ed. L S Stil'bans (Plenum Press, New York)

[175] Broido D A and Reinecke T L (2001) Phys Rev B 64045324

[176] Chen X, Parker D and Singh D J (2013) Sci Rep 33168

[177] Zhang G, Kirk B, Jauregui L A, Yang H, Xu X, Chen Y P and Wu Y (2012) Nano Lett 1256

[178] Balandin A and Kang K L (1998) J Appl Phys 846149

[179] Yelgel Ö C and Srivastava G P (2014) Phil Mag 84 (in production)

[180] Anderson D L (1959) J Phys Chem Solids 1241

[181] Thomas I O and Srivastava G P 2014 AIP Conf Proc 159095

[182] Van de Walle C G and Martin R M 1986 Phys Rev B 345621 